Singularidades no infinito de funções polinomiais

Nilva Rodrigues Ribeiro 
SERVIÇO DE PÓS-GRADUAÇ̃̃O DO ICMC-USP

Data de Depósito: 07/12/2012

Assinatura:

\title{
Singularidades no infinito de funções polinomiais
}

\author{
Nilva Rodrigues Ribeiro
}

Orientadora: Profa. Dra. Maria Aparecida Soares Ruas Co-orientador: Prof. Dr. Raimundo Nonato Araújo dos Santos

Tese apresentada ao Instituto de Ciências Matemáticas e de Computação - ICMC-USP, como parte dos requisitos para obtenção do título de Doutor em Ciências - Matemática . VERSÃO REVISADA. 
Ficha catalográfica elaborada pela Biblioteca Prof. Achille Bassi e Seção Técnica de Informática, ICMC/USP, com os dados fornecidos pelo(a) autor(a)

Ribeiro, Nilva Rodrigues
Singularidades no infinito de funções polinomiais
/ Nilva Rodrigues Ribeiro; orientadora Maria
Aparecida Soares Ruas; co-orientador Raimundo
Nonato Araújo dos Santos. -- São Carlos, 2012.
122 p.
Tese (Doutorado - Programa de Pós-Graduação em
Matemática)-- Instituto de Ciências Matemáticas e
de Computação, Universidade de São Paulo, 2012.
1. Classificação. 2. Singularidades. 3. Funções
polinomiais. I. Ruas, Maria Aparecida Soares,
orient. II. Araújo dos Santos, Raimundo Nonato, co-
orient. III. Título.


"Agradeço pelas inúmeras vezes que você me enxergou melhor do que eu sou. Pela sua capacidade de me olhar devagar, já que nessa vida muita gente me olhou depressa demais" Pe. Fabio de Mello 
À minha família,

dedico. 


\section{Agradecimentos}

Este trabalho é para mim mais que uma tese de doutorado. É a concretização de esforços não só meus, mas de muitas outras pessoas especiais a quem começo agradecer.

Primeiramente a Deus pelo precioso dom da vida. Obrigada Senhor, por ter me sustentado por todo este caminho, me dando força e ânimo. À Mãe do céu, por sempre interceder por esta sua filha, obrigada pela proteção constante.

À professora Maria Aparecida Soares Ruas e ao professor Raimundo Nonato dos Santos, por terem me orientado durante este período, pela paciência, dedicação e pelo constante incentivo, sempre indicando a direção a ser tomada nos momentos de maior dificuldades. Com eles, aprendi muito tanto do ponto de vista matemático, como do ponto de vista humano.

À $U F V / M G$, por ter proporcionado condições para a realização deste projeto.

Aos colegas de pós-graduação, pelo convívio e aos professores e funcionários do ICMC/USP por toda disponibilidade em ajudar, sempre que necessário.

A todos os profissionais, seja do ensino primário, fundamental, médio ou do ensino superior. Obrigada por fazer parte desta conquista.

À minha mãe Iracema, exemplo de vida. Por todo amor, compreensão e apoio durante esta caminhada e por entender anos de ausência.

Ao meu pai Emídio Paulo ( in memorian), que também estaria feliz co- 
migo pela conquista deste título se estivesse ainda entre nós.

Aos meus irmãos(âs) com suas(seus) respectivas(os) esposas(os): Zama e M. Ilda, Maria, Joaquim e Vera, Lázaro e Edilene, Aparecida e João, Mauro e Lourdes, pelo companherismo de uma vida, que apesar da distância estiveram presentes a cada instante.

À Fabiana irmã de coração, sem sua ajuda amiga, seria muito mais difícil chegar até aqui.

Talvez nem saibam a sua importância em minha vida, meus sobrinhos: Nai, Maisa e Carlos, Marcos, Frank, Laurinha, Ma, Ló, Isa e Lipe. Alegrias incomparáveis ao lado deles.

Ao Paulo, uma pessoa tão especial em minha vida. Por todo apoio, carinho e paciência.

À toda minha família, tios, primos e amigos. A vida se torna melhor com todas as histórias, conversas, almoços e "salgadinhos" nos fins de semana.

Peço desculpas as pessoas que contribuíram direta ou indiretamente na realização deste projeto e não as citei, mas agradeço a todos.

Finalmente, peço a Deus que os abençoe.

Obrigada! 


\section{Resumo}

O principal objetivo desta tese é classificar as singularidades no infinito de polinômios em $\mathbb{C}^{n}$. Aplicamos inicialmente o método utilizado por Siersma e Smeltink em [38], para classificar polinômios de grau $3 \mathrm{em} \mathbb{C}^{3}$. Este método consiste em classificar polinômios fixando uma forma normal para a parte homogênea de maior grau. As singularidades no infinito de funções polinomiais podem ser estudadas através das singularidades das homogenizações destas aplicações definidas no espaço projetivo. Este é o método utilizado por Bruce e Wall em [11], que fazem uma classificação das superfícies cúbicas no espaço projetivo $\mathbb{P}^{3}$, relacionando as singularidades destas superfícies com a classificação de certos sistemas polinomiais a elas associados. Um dos objetivos do nosso trabalho é estender parcialmente o método de Bruce e Wall para classificar as singularidades no infinito de polinômios $f=f_{d-1}+f_{d}$ em $\mathbb{C}^{n}$, com $d \geq 3$, através do estudo das singularidades do sistema polinomial $g=\left(f_{d-1}, f_{d}\right)$. Para polinômios de grau $3 \mathrm{em} \mathbb{C}^{3}$, fazemos um refinamento das formas normais de [11], que possibilita uma descrição mais detalhada da fibra especial e o estudo no infinito da topologia da fibra genérica. Isto é feito com o auxílio do invariante $\Delta_{n-1}(f)$ definido por Siersma e Tibar em [39], e por eles denominado defeito maximal de Betti. 


\begin{abstract}
The main purpose of this thesis is to classify singularities at infinity of polynomial functions $f: \mathbb{C}^{n} \rightarrow \mathbb{C}$. We first apply Siersma and Smeltink's method [38] to classify degree 3 polynomials in $\mathbb{C}^{3}$. This method consists on classifying polynomials fixing the normal form of their highest homogeneous part. The singularities at infinity of polynomial functions may also be studied through the classification of singularities of the projective hypersurfaces $F=0$, where $F$ is the homogenization of $f$. This was the method applied by Bruce and Wall in [11], in their classification of the cubic surfaces in $\mathbb{P}^{3}$. They relate the singularities of the cubic surfaces with the singularities of certain systems of polynomials. In our work, we partially extend Bruce and Wall's method to classify the singularities at infinity of polynomials $f=f_{d-1}+f_{d}$ in $\mathbb{C}^{3}, n \geq 3$, based on the investigation of singularities of the polynomial system $g=\left(f_{d-1}, f_{d}\right)$. For the class of degree 3 polynomials in $\mathbb{C}^{3}$, we refine Bruce-Wall's classification, in order to present a more detailed description of the special fiber of $f$ and to investigate its topology with the help of the invariant Betti maximal defect, introduced by Siersma and Tibar in [39].
\end{abstract}




\section{Sumário}

Referências Bibliográficas $i$

1 Preliminares 5

1.1 Germes e k-Jatos . . . . . . . . . . . . . 5

1.2 Ação de grupos. . . . . . . . . . . . . . . . . . 6

1.3 A Álgebra $\mathcal{O}_{n} \ldots \ldots \ldots \ldots 7$

1.4 Relações de equivalência no espaço dos germes de aplicações 8

1.5 Classificação de germes de funções . . . . . . . . . . . . . . 10

1.6 Desdobramentos e deformações . . . . . . . . . . . . . . 12

1.7 Singularidades simples . . . . . . . . . . . . . . 13

1.8 Singularidades não isoladas . . . . . . . . . . . . . . . 16

2 Estratificação e interseção completa 19

2.1 Conjuntos algébricos, semialgébricos e variedades analíticas . 19

2.2 Estratificações . . . . . . . . . . . . . . . . . . . . 22

2.3 Interseção completa . . . . . . . . . . . . . . . . . 25

3 Singularidades no infinito 27

3.1 Germes de funções com singularidade isolada . . . . . . . . 28

3.2 Singularidades no infinito . . . . . . . . . . . . . . . . . 29

3.2.1 Como encontrar valores atipicos . . . . . . . . . . 30 
4 Classificação de polinômios de grau 3 em $\mathbb{C}^{3}$

4.1 Introdução . . . . . . . . . . . . . . . . 35

4.2 Cúbicas em $\mathbb{P}^{2} \ldots \ldots \ldots \ldots$. . . . . . . . . . . 36

4.2.1 Forma cúbica não singular em $\mathbb{P}^{2}$. . . . . . . . . 36

4.2.2 Formas cúbicas singulares em $\mathbb{P}^{2}$. . . . . . . . 37

4.2.3 Classificação de polinômios de grau $3 \mathrm{em} \mathbb{P}^{3}$. . . . . 40

4.3 Classificação projetiva de F . . . . . . . . . . . . . . . . . 41

4.3.1 $f_{3}$ nodal . . . . . . . . . . . . . . . . . 41

4.3.2 $f_{3}$ cuspidal ....................... 43

4.3.3 $f_{3}$ cônica mais corda. . . . . . . . . . . . . . . 44

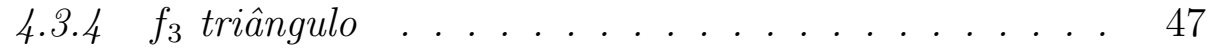

4.3.5 $f_{3}$ cônica mais tangente . . . . . . . . . . . 48

4.3.6 $f_{3}$ três retas concorrentes . . . . . . . . . . . . 49

4.3.7 $f_{3}$ duas retas, uma com multiplicidade $2 \ldots . . . .50$

4.3.8 $f_{3}$ uma reta de multiplicidade 3 . . . . . . . . . . 52

4.4 Regularidade no infinito . . . . . . . . . . . . . . 53

5 Classificação de superfícies cúbicas projetivas 61

5.1 Introdução . . . . . . . . . . . . . . . . . 61

5.2 Cúbicas singulares em $\mathbb{P}^{3} \ldots \ldots . \ldots . \ldots 61$

5.3 P é nó cônico . . . . . . . . . . . . . . . . . . . . . 62

5.3.1 Descrevendo a classificação . . . . . . . . . . . . 66

5.3.2 Refinamento da classificação quando $f_{2}$ é nó cônico . 68

5.4 P é um binó . . . . . . . . . . . . . . . . . . . . . . 71

5.4.1 Descrevendo a classificação . . . . . . . . . . . 73

5.4.2 Refinamento da classificação quando $f_{2}$ é binó . . . . 77

5.5 P é nó degenerado . . . . . . . . . . . . . . . . . . . . . 81

5.5.1 Refinamento da classificação quando $f_{2}$ é nó degenerado 82 
6 Defeito de Betti de polinômios de grau 3 em $\mathbb{C}^{3} \quad 91$

6.1 Introdução . . . . . . . . . . . . . . . . . . . . . . 91

6.2 Deformaçôes de polinômios . . . . . . . . . . . . . . . . . 92

6.3 Defeito de Betti de polinômios de grau $3 \mathrm{em} \mathbb{C}^{3}$. . . . . . 96

7 Feixes homogêneos e classificação de funções polinomiais 103 7.1 Introdução . . . . . . . . . . . . . . . . . . . . . 103

7.2 Feixes homogêneos e classificação de aplicações polinomiais . 104

7.3 Superfícies em $\mathbb{C}^{3}$ com defeito de Betti mínimo e máximo . . 110

Referências Bibliográficas 117 


\section{Introdução}

$O$ estudo de fibrações singulares de um polinômio $f: \mathbb{C}^{n} \rightarrow \mathbb{C}$ foi introduzido por Broughton [9] há mais de 20 anos. Ao mesmo tempo, Pham [35] estudou condições para que um polinômio tivesse um bom comportamento no infinito, e Hà e Lê [20] obtiveram um critério para detectar valores atípicos em polinômios complexos de duas variáveis. Desde então uma ampla teoria global de singularidades de polinômios tem sido desenvolvida, do ponto de vista desta tese, com contribuições de [49], [50], [40], [13], [22], entre outros.

No estudo local, a presença de uma singularidade é a obstrução natural para a existência de uma fibração trivial associada a um germe f. No contexto global, entretanto, como mostra o exemplo de Broughton, as fibras de um polinômio podem ser topologicamente distintas, mesmo sem a presença de singularidades. Os valores de $f$ para os quais a topologia da fibra muda são denominados valores atípicos e a determinação destes valores especiais depende do comportamento de $f$ no infinito.

No caso de polinômios de duas variáveis, diversas caracterizações das singularidades no infinito ([13]) são conhecidas, enquanto que em dimensões mais altas, o problema de caracterizar valores atípicos é um problema aberto.

Uma evidência do impacto das singularidades no infinito é a sua conexão com a Conjectura Jacobiana. Com efeito, esta conjectura é equivalente à seguinte formulação ([24], [40]):

"Se $f: \mathbb{C}^{2} \rightarrow \mathbb{C}$ não tem pontos críticos, mas tem singularidades no infinito, então para qualquer polinômio $h: \mathbb{C}^{2} \rightarrow \mathbb{C}$, o conjunto singular de $(f, h): \mathbb{C}^{2} \rightarrow \mathbb{C}^{2}$ é não vazio."

Assim como no caso local, o número de Milnor no infinito e a soma dos números de Milnor da fibra genérica são invariantes úteis para o estudo da topologia da fibra. 
Uma abordagem para este estudo consiste em classificar as aplicações polinomiais e procurar informações sobre a topologia das fibras em cada classe de equivalência. A classificação global das singularidades de aplicações polinomiais é um problema difícil e poucos são os métodos conhecidos.

O principal objetivo desta tese é classificar as singularidades no infinito de polinômios em $\mathbb{C}^{n}$. Aplicamos inicialmente o método utilizado por Siersma e Smeltink em [38], para classificar polinômios de grau $3 \mathrm{em} \mathbb{C}^{3}$. Este método consiste em classificar polinômios fixando uma forma normal para a parte homogênea de maior grau.

As singularidades no infinito de aplicações polinomiais podem ser estudadas através das singularidades das homogenizações destas aplicações definidas no espaço projetivo. Este é o método utilizado por Bruce e Wall em [11], que fazem uma classificação das superfícies cúbicas no espaço projetivo $\mathbb{P}^{3}$, relacionando as singularidades destas superfícies com a classificação de certos sistemas polinomiais a elas associados. Um dos objetivos do nosso trabalho é estender parcialmente o método de Bruce e Wall para classificar as singularidades no infinito de polinômios $f=f_{d-1}+f_{d}$ em $\mathbb{C}^{n}$, com $d>3$, através do estudo das singularidades do sistema polinomial $g=\left(f_{d-1}, f_{d}\right)$.

Para polinômios de grau $3 \mathrm{em} \mathbb{C}^{3}$, fazemos um refinamento das formas normais de [11], que possibilita uma descrição mais detalhada da fibra especial e o estudo no infinito da topologia da fibra genérica. Isto é feito com o auxilio do invariante $\Delta_{n-1}(f)$ definido por Siersma e Tibar em [39], e por eles denominado defeito maximal de Betti.

No capítulo 1, apresentamos definições e resultados básicos da teoria local de singularidades, tais como, germes de aplicações, ação de grupos, determinação finita e deformação de singularidades. Complementamos o capítulo com um estudo de germes simples com singularidade não-isolada 
1-dimensional.

No capítulo 2, apresentamos resultados básicos da teoria de estratificação de Thom-Whitney. Estudamos também propriedades das interseções completas.

O capítulo 3 apresenta definições e resultados importantes para o estudo de singularidades no infinito de aplicações polinomiais, como o Teorema do Bouquet de M. Tibar [48].

Em [38], Siersma e Smeltink classificaram as singularidades no infinito de polinômios de grau 4 em 2 variáveis, obtendo condições para a equivalência de polinômios que possuem partes homogêneas de grau 4 equivalentes. No Capítulo 4, utilizamos este método para classificar as singularidades no infinito de polinômios $f: \mathbb{C}^{3} \rightarrow \mathbb{C}$ de grau 3 em 3 variáveis, $f\left(x_{0}, x_{1}, x_{2}\right)=f_{1}\left(x_{0}, x_{1}, x_{2}\right)+f_{2}\left(x_{0}, x_{1}, x_{2}\right)+f_{3}\left(x_{0}, x_{1}, x_{2}\right), f_{i}$ polinômio homogêneo de grau $i, i=1,2,3$. Relacionamos o tipo de singularidade de $f$ com a singularidade de $f_{3}$ e finalizamos estudando o comportamento topológico da fibra genérica no infinito.

No capítulo 5, nossa atenção será voltada para o caso particular das funções polinomiais $f: \mathbb{C}^{3} \rightarrow \mathbb{C}$ de grau 3 em 3 variáveis, $f\left(x_{0}, x_{1}, x_{2}\right)=$ $f_{2}\left(x_{0}, x_{1}, x_{2}\right)+f_{3}\left(x_{0}, x_{1}, x_{2}\right), f_{i}$ polinômio homogêneo de grau $i, i=2,3$. Apresentamos um refinamento da classificação de Bruce e Wall em termos das singularidades de $f_{3}$. Esta classificação será importante no estudo da topologia da fibra, que será apresentado no capítulo 6.

No capitulo 6, apresentamos resultados novos da classificação das singularidades no infinito de polinômios de grau $3 \mathrm{em} \mathbb{C}^{3}$, relacionando o tipo topológico da fibra no infinito com o invariante defeito maximal de Betti definido por Siersma e Tibar em [39].

No capítulo 7, generalizamos alguns resultados de [11] para uma função 
polinomial da forma $f: \mathbb{C}^{n} \rightarrow \mathbb{C}$ de grau d em $n$ variáveis, $f(x)=f_{1}(x)+$ $\ldots+f_{d}(x), x \in \mathbb{C}^{n}, f_{i}$ polinômio homogêneo de grau $i$. O principal resultado é o Teorema 7.5 em que relacionamos a classificação das singularidades no infinito de $f=f_{d-1}+f_{d}$ com as singularidades do sistema $g=\left(f_{d-1}, f_{d}\right)$.

O método é aplicado no estudo das singularidades no infinito de classes especiais de polinômios em $\mathbb{C}^{3}$ que possuem apenas uma singularidade no infinito, que denominamos "nó generalizado". No Teorema 7.13 caracterizamos completamente nós generalizados de defeito de Betti mínimo. No Teorema 7.14, apresentamos uma caracterização parcial de nós generalizados com defeito máximo. Estudamos também o caso em que o conjunto singular de $f$ em $\mathbb{C}^{3}$ é não isolado. 


\section{Capítulo 1}

\section{Preliminares}

Neste Capítulo vamos relembrar algumas definições e resultados clássicos necessários para o desenvolvimento desta tese. Iniciamos introduzindo germes de aplicações analíticas e a álgebra $\mathcal{O}_{n}$. Posteriormente, apresentamos resultados sobre a classificação de germes de funções. As principais referências são [10], [17], [16], [42], [36] e [28].

\subsection{Germes e k-Jatos}

Definição 1.1. Sejam $x \in \mathbb{K}^{n}, f: U_{1} \subset \mathbb{K}^{n} \rightarrow \mathbb{K}^{p}$ e $g: U_{2} \subset \mathbb{K}^{n} \rightarrow \mathbb{K}^{p}$ aplicações analíticas definidas em vizinhanças abertas $U_{1}$ e $U_{2}$ de $x$, em que $\mathbb{K}=\mathbb{R}$ ou $\mathbb{C}$. Dizemos que $f$ e $g$ são equivalentes se existir uma vizinhança de $x$ em $\mathbb{K}^{n}, U \subset U_{1} \cap U_{2}$ tal que $\left.f\right|_{U}=\left.g\right|_{U}$. As classes de equivalência sob essa relação são chamadas de germes de aplicação analítica de $\mathbb{K}^{n}$ em $\mathbb{K}^{p}$ em $x$. Os elementos de uma classe são chamados representantes do germe ( usamos a notação $f:\left(\mathbb{K}^{n}, x\right) \rightarrow \mathbb{K}^{p}$ para um germe de $f$ em $x$ ). O ponto $x \in \mathbb{K}^{n}$ é chamado fonte do germe $f$. No estudo local das singularidades, por uma translação em $\mathbb{K}^{n}$, podemos supor $x=0$.

O germe de uma aplicação $f:\left(\mathbb{K}^{n}, 0\right) \rightarrow \mathbb{K}^{p}$ é singular para algum re- 
presentante da classe digamos $f$, se a matriz Jacobiana de $f$ em 0 tem rank menor que o mínimo entre $n$ e $p$, caso contrário, dizemos que o germe é regular. É fácil ver que a definição acima não depende da escolha do representante.

Definição 1.2. O espaço de jatos $J^{k}(n, p)$ é o espaço vetorial das aplicações $f: \mathbb{K}^{n} \rightarrow \mathbb{K}^{p}$ em que cada componente $f_{i}$ de $f$ é um polinômio de grau menor ou igual a $k$ nas coordenadas canônicas de $\mathbb{K}^{n}$ com termos constantes nulos. Os elementos de $J^{k}(n, p)$ são chamados $k$-jatos.

\subsection{Ação de grupos}

Sejam $G$ um grupo e $M$ um conjunto. Uma ação de $G$ em $M$ é uma aplicação $\phi: G \times M \rightarrow M$, denotada por $\phi(g, x)=g . x$ satisfazendo:

i) $1 . \mathrm{x}=\mathrm{x}$,onde 1 é a identidade de $G$.

ii) $(g h) \cdot x=g \cdot(h . x), \forall x \in M, \forall g, h \in G$.

Dada uma ação podemos definir uma relação de equivalência em $M$. Se $x, y \in M, x$ é equivalente a $y$ se existe $g \in G$ tal que $y=g . x$. As classes de equivalência são chamadas de órbitas. Se $x \in M$, a órbita de $x$ é o conjunto

$$
G \cdot x=\{g \cdot x \quad / g \in G\}
$$

Definição 1.3. Um grupo de Lie $G$ é um grupo que é uma variedade diferenciável e as aplicações de multiplicação $G \times G \rightarrow G,\left(g_{1}, g_{2}\right) \mapsto g_{1} g_{2} e$ inversão $G \rightarrow G, g \mapsto g^{-1}$, são de classe $\mathbb{C}^{\infty}$.

Lema 1.4. (Mather) Seja $G$ um grupo de Lie agindo numa variedade M. Seja $N \subset M$ uma subvariedade conexa e suponhamos que as órbitas são subvariedades de $M$. Então $N$ está contida em uma única órbita, se e 
somente se:

1) $T_{y} G . y \supset T_{y} N, \forall y \in N$.

2) $\operatorname{dim}_{y} G . y=k, \forall y \in N$, onde $k$ é uma constante.

A prova do Lema 1.4 pode ser encontrada em [16].

\subsection{A Álgebra $\mathcal{O}_{n}$}

Definimos a álgebra local dos germes analíticos em $\mathbb{K}^{n}$

$$
\mathcal{O}_{n}=\left\{f:\left(\mathbb{K}^{n}, 0\right) \rightarrow \mathbb{K} \mid \text { tal que } f \text { é um germe de função analítica }\right\} \text {. }
$$

Temos que $\mathcal{O}_{n}$ é um anel local cujo ideal maximal é

$$
\mathcal{M}_{n}=\left\{f \in \mathcal{O}_{n} \mid f(0)=0\right\} .
$$

O conjunto dos germes analíticos $f:\left(\mathbb{K}^{n}, 0\right) \rightarrow \mathbb{K}^{p}$, denotado por

$$
\mathcal{O}_{n, p}=\left\{f:\left(\mathbb{K}^{n}, 0\right) \rightarrow \mathbb{K}^{p} \mid \text { tal que } f \text { é uma aplicação analítica }\right\}
$$

é um $\mathcal{O}_{n}$-módulo livre de posto $p$.

O submódulo dos germes que satisfazem $f(0)=0$ é dado por

$$
\mathcal{O}_{n, p}^{0}=\left\{f \in \mathcal{O}_{n, p} \mid f(0)=0\right\}
$$

Lema 1.5. (Nakayama) Sejam $\mathcal{R}$ um anel comutativo com identidade $1 e$ $\mathcal{M}$ um ideal de $\mathcal{R}$ tal que $1+x$ é invertível em $\mathcal{R}, \quad \forall x \in \mathcal{M}$. Sejam $M$ um $\mathcal{R}$-módulo, $A$ e $B \mathcal{R}$-submódulos com A finitamente gerado. Se $A \subset B+\mathcal{M} A$ então $A \subset B$.

A prova do Lema 1.5 pode ser encontrada em [16]. 


\subsection{Relações de equivalência no espaço dos germes de aplicações}

Para mais detalhes desta subseção, veja [16].

As relações de equivalências mais utilizadas para o problema de classificação de germes de aplicações analíticas são as $G$-equivalências, onde $G$ é um dos grupos de Mather $\mathcal{R}, \mathcal{L}, \mathcal{C}, \mathcal{K}$ ou $\mathcal{A}$. Dentre estes, os que nos interessam são os grupos $\mathcal{R}, \mathcal{C}$ e $\mathcal{K}$.

Definição 1.6. Denotaremos por $\mathcal{R}$ o grupo dos germes de difeomofismos analíticos, $h:\left(\mathbb{K}^{n}, 0\right) \rightarrow\left(\mathbb{K}^{n}, 0\right)$.

Este grupo é chamado grupo de mudanças de coordenadas na fonte e age sobre $\mathcal{O}_{n, p}$ por composição à direita

$$
\begin{aligned}
& \mathcal{R} \times \mathcal{O}_{n, p} \rightarrow \mathcal{O}_{n, p} \\
& (h, f) \longmapsto f \circ h^{-1}
\end{aligned}
$$

Definição 1.7. Sejam $f, g \in \mathcal{O}_{n, p}$. Dizemos que $f$ é $\mathcal{R}$-equivalente a $g$ se existe $h \in \mathcal{R}$ tal que $g=f \circ h^{-1}$.

Notação: $f \stackrel{\mathcal{R}}{\sim} g$

Definição 1.8. Seja $f \in \mathcal{O}_{n, p}$, tal que $f(0)=0$. Definimos $f^{\star}: \mathcal{O}_{p} \rightarrow \mathcal{O}_{n}$ por $f^{\star}(g)=g \circ f$ e dizemos que $f^{*}$ é o homomorfismo induzido por $f$.

Definição 1.9. O grupo de contato que denotaremos por $\mathcal{K}$, é o grupo dos germes de difeomorfismo $\left(\mathbb{K}^{n} \times \mathbb{K}^{p}, 0\right) \rightarrow\left(\mathbb{K}^{n} \times \mathbb{K}^{p}, 0\right)$ que são da forma

$$
H(x, y)=(h(x), \theta(x, y))
$$

onde $h \in \mathcal{R}$ e $\theta(x, 0)=0$, para $x$ próximo da origem. 
Definição 1.10. Sejam $f, g \in \mathcal{O}_{n, p}^{0}$. Dizemos que $f$ e $g$ são $\mathcal{K}$-equivalentes se existe $H=(h, \theta) \in \mathcal{K}$ tal que $H(x, f(x))=(h(x), g(h(x)))$.

Isto é, $H$ leva o gráfico de $f$ sobre o gráfico de $g$, deixando $\mathbb{K}^{n} \times\{0\}$ invariante.

Notação: $f \stackrel{\mathcal{K}}{\sim} g$

Nota 1.11. Se $f \stackrel{\mathcal{K}}{\sim} g$, então os conjuntos $f^{-1}(0)$ e $g^{-1}(0)$ são isomorfos. Mas precisamente, se existe $H=(h, \theta) \in \mathcal{K}$ tal que $H(x, f(x))=$ $(h(x), g(h(x)))$, então $h\left(f^{-1}(0)\right)=g^{-1}(0)$.

Denotaremos por $\mathcal{C}$ o subgrupo de $\mathcal{K}$ formado pelos elementos da forma $H=\left(i_{d_{n}}, \theta\right)$, onde $i_{d_{n}}$ é a aplicação identidade de $\mathbb{K}^{n}$. Da mesma forma, temos que dois germes $f, g \in \mathcal{O}_{n, p}^{0}$ são $\mathcal{C}$-equivalentes quando existir $H \in \mathcal{C}$ tal que

$$
H(x, f(x))=(x, \theta(x, f(x)))=(x, g(x)) .
$$

Nota 1.12. Podemos ver $\mathcal{K}$ como o produto semi-direto dos grupos $\mathcal{R}$ e $\mathcal{C}$, $\operatorname{ver}([16], p .150)$.

Lema 1.13. Dois germes $f$ e $g$ são $\mathcal{K}$-equivalentes, se e somente se, existe um germe inversivel $h:\left(\mathbb{K}^{n}, 0\right) \rightarrow\left(\mathbb{K}^{n}, 0\right)$ para o qual os germes $f \circ h$ e $g$ são $\mathcal{C}$-equivalentes.

Proposição 1.14. ([16]) Sejam $f, g \in \mathcal{O}_{n, p}^{0}$. As seguintes condições são equivalentes:

(i) $f \stackrel{\mathcal{C}}{\sim} g$

(ii) $I(f)=I(g)$, onde $I(f)=<f_{1}, \ldots, f_{p}>\subset \mathcal{O}_{n}$ é o ideal gerado por 
$f_{1}, \ldots, f_{p}$.

(iii) Existe uma matriz $p \times p$ inversivel $U=\left(u_{i, j}\right)_{p \times p}, u_{i, j} \in \mathcal{O}_{n}$ tal que $f_{i}(x)=\sum_{j=1}^{p} u_{i, j}(x) g_{j}(x), \quad i=1, \ldots, p$.

Definição 1.15. (i) A álgebra local de um germe $f:\left(\mathbb{K}^{n}, 0\right) \rightarrow\left(\mathbb{K}^{p}, 0\right)$ é definida por

$$
\mathcal{O}_{n}=\frac{\mathcal{O}_{n}}{f^{*} \mathcal{M}_{p}}
$$

(ii) A multiplicidade local de um germe de aplicação $f \in \mathcal{O}_{n, p}, n \leq p$ é

$$
m_{p}(0)=\operatorname{dim}_{\mathbb{K}} \frac{\mathcal{O}_{n}}{f^{*} \mathcal{M}_{p}}
$$

\subsection{Classificação de germes de funções}

Nesta subseção vamos particularizar para o caso $p=1$.

Para um germe $f:\left(\mathbb{K}^{n}, 0\right) \rightarrow(\mathbb{K}, 0)$ definimos o ideal jacobiano de $f$ como o ideal gerado pelas derivadas parciais, $J f=\left(\frac{\partial f}{\partial x_{1}}, \ldots, \frac{\partial f}{\partial x_{n}}\right)$.

Definição 1.16. Seja $f \in \mathcal{O}_{n}$.

1) $O$ espaço tangente a $f$ segundo o grupo $\mathcal{R}$ é definido por $T \mathcal{R} f=\mathcal{M}_{n} J f$.

2) O espaço tangente estendido a $f$ segundo o grupo $\mathcal{R}$, é definido por $T \mathcal{R}_{e} f=J f$.

3) A $\mathcal{R}$-codimensão de $f$ é a dimensão de $\frac{\mathcal{M}_{n}}{\mathcal{M}_{n} J f}$ como espaço vetorial real e é denotada por $\mathcal{R}-\operatorname{codf}$.

4) $A \mathcal{R}_{e}$-codimensão de $f$ é a dimensão de $\frac{\mathcal{O}_{n}}{J f}$ como espaço vetorial real 
e é denotada por $\mathcal{R}_{e}-\operatorname{codf}$. A dimensão de $\frac{\mathcal{O}_{n}}{J f}$, quando finita, é o conhecido número de Milnor [30].

Definição 1.17. Sejam $f, g \in J^{k}(n, 1)$. Dizemos que $f$ e $g$ são $\mathcal{R}^{k}$ - equivalentes, se existe $h \in \mathcal{R}$ tal que $J^{k} f(0)=J^{k}\left(f \circ h^{-1}\right)(0)$. O espaço tangente à órbita de $f$ em $J^{k}(n, 1)$ é definido por

$$
T \mathcal{R}^{k} \cdot f=\frac{\mathcal{M}_{n} J f}{\mathcal{M}_{n}^{k+1}}
$$

Temos assim que $\mathrm{TR}^{k} f$ é gerado por $k$-jatos de elementos de $\mathcal{M}_{n} J f$.

Proposição 1.18. Seja $f \in \mathcal{O}_{n}$. Então $\mathcal{R}-\operatorname{codf}<\infty$, se e somente se, existe um inteiro $k>0$ tal que $J f \supset \mathcal{M}_{n}^{k}$, ou equivalentemente $\mathcal{M}_{n}^{k} \subset$ $J f+\mathcal{M}_{n}^{k+1}$.

Proposição 1.19. Seja $f \in \mathcal{O}_{n}$ tal que $0<\mathcal{R}-\operatorname{codf}<\infty$. Então

$$
\mathcal{R}-\operatorname{codf}=\mathcal{R}_{e}-\operatorname{codf}+n-1 .
$$

Definição 1.20. Dizemos que $f \in \mathcal{O}_{n}$ é $k$-determinado se todo $g \in \mathcal{O}_{n}$ tal que $J^{k} f(0)=J^{k} g(0)$ é $\mathcal{R}$-equivalente a $f$. Dizemos que $f$ é finitamente determinado se é $k$-determinado para algum $k$.

Proposição 1.21. Se $f \in \mathcal{O}_{n}$ é tal que $\mathcal{M}_{n}^{k} \subset \mathcal{M}_{n} J f$, então $f$ é $k$ determinado.

Proposição 1.22. Seja $f \in \mathcal{M}_{n}$. Se $f$ é $k$-determinado então $\mathcal{M}_{n} J f \supset$ $\mathcal{M}_{n}^{k+1}$.

Proposição 1.23. Sejam $f, g \in \mathcal{O}_{n}$, Se $f$ e g são $\mathcal{R}$-equivalentes e $f$ é $k$-determinado então $g$ é $k$-determinado. 
Definição 1.24. Dizemos que um germe $f \in \mathcal{M}_{n}$ tem uma singularidade não degenerada em 0 se:

1) $d f(0)=0$, isto é, $\frac{\partial f}{\partial x_{i}}(0)=0, \forall i=1, \ldots, n$.

2) $\operatorname{det}\left(\frac{\partial^{2} f}{\partial x_{i} x_{j}}(0)\right) \neq 0$, ou seja o rank da matriz $\left(\frac{\partial^{2} f}{\partial x_{i} x_{j}}(0)\right)$ é $n$.

Nesse caso dizemos que $f$ é Morse ou tem uma singularidade de Morse.

Proposição 1.25. Seja $f \in \mathcal{M}_{n}$. Então $f$ tem uma singularidade não degenerada em 0 , se e somente se, $J f=\mathcal{M}_{n}$.

Lema 1.26. (Morse) Se $f \in \mathcal{M}_{n}$ tem uma singularidade não degenerada em 0 , então existe $h \in \mathbb{K}^{n}$ tal que:

$$
\left(f \circ h^{-1}\right)\left(x_{1}, \ldots, x_{n}\right)=-x_{1}^{2}-\ldots-x_{r}^{2}+x_{r+1}^{2}+\ldots+x_{n}^{2} .
$$

\subsection{Desdobramentos e deformações}

Definição 1.27. Seja $f_{0} \in \mathcal{M}_{n} \mathcal{O}_{n, p}$. Um desdobramento a s-parâmetros de $f_{0}$ é um germe $F:\left(\mathbb{K}^{n} \times \mathbb{K}^{s},(0,0)\right) \rightarrow\left(\mathbb{K}^{p} \times \mathbb{K}^{s},(0,0)\right)$ dado por $(x, u) \mapsto$ $(f(x, u), u)$ tal que $f(x, 0)=f_{0}(x)$. O germe $f:\left(\mathbb{K}^{n} \times \mathbb{K}^{s},(0,0)\right) \rightarrow\left(\mathbb{K}^{p}, 0\right)$ é chamado deformação de $f_{0}$.

No caso em que $p=1$ temos:

Definição 1.28. Duas deformações $f$ e $g$ a s-parâmetros de $f_{0}$ são isomorfas se existir um germe de difeomorfismo $\Phi:\left(\mathbb{K}^{n} \times \mathbb{K}^{s}, 0\right) \rightarrow\left(\mathbb{K}^{n} \times \mathbb{K}^{s}, 0\right)$ tal que:

1) $\phi(x, u)=(\psi(x, u), u)$ tal que $\psi(x, 0)=x$.

2) $g=f \circ \phi$. 
Definição 1.29. Uma deformação é trivial se é isomorfa à deformação constante dada por $(x, u) \mapsto f_{0}(x)$.

Seja $f$ uma deformação a s-parâmetros de $f_{0} \in \mathcal{M}_{n}$. Seja $h:\left(\mathbb{K}^{q}, 0\right) \rightarrow$ $\left(\mathbb{K}^{s}, 0\right)$ germe de aplicação analítica. Definimos $g:\left(\mathbb{K}^{n} \times \mathbb{K}^{s}, 0\right) \rightarrow(\mathbb{K}, 0)$ por $g(x, u)=f(x, h(u))$. Assim $g$ é uma deformação a q-parâmetros de $f_{0}$ que denotamos por $g=h^{*} f$. A deformação $h^{*} f$ é chamada pull-back de $f$ por $h$.

Definição 1.30. Duas deformações $f$ e $g$ a s-parâmetros são equivalentes se existir um germe de difeomorfismo $h:\left(\mathbb{K}^{s}, 0\right) \rightarrow\left(\mathbb{K}^{s}, 0\right)$ tal que $f$ é isomorfo $a h^{*} f$.

Definição 1.31. Se $\bar{g}$ é uma deformação a q-parâmetros, dizemos que $\bar{g}$ é induzida de $f$ se existir um germe $:\left(\mathbb{K}^{q}, 0\right) \rightarrow\left(\mathbb{K}^{s}, 0\right)$ tal que $\bar{g}$ é isomorfo a $k^{*} f$.

Definição 1.32. Uma deformação $f$ de $f_{0} \in \mathcal{M}_{n}$ é versal se qualquer outra deformação de $f_{0}$ é induzida de $f$.

Dizemos que $f$ é miniversal se for versal com o número mínimo de parâmetros.

Definição 1.33. Se $f:\left(\mathbb{K}^{n} \times \mathbb{K}^{s}, 0\right) \rightarrow(\mathbb{K}, 0)$ é uma deformação de $f_{0} \in$ $\mathcal{M}_{n}$ então as velocidades iniciais $\dot{f}_{i} \in \mathcal{O}_{n}, i=1, \ldots, s$ da deformação são definidas por $\dot{f}_{i}=\frac{\partial f}{\partial u_{i}}(x, 0)$.

Proposição 1.34. Temos que $f:\left(\mathbb{K}^{n} \times \mathbb{K}^{s}, 0\right) \rightarrow(\mathbb{K}, 0)$ é uma deformação versal de $f_{0} \in \mathcal{M}_{n}$, se e somente se, a $\mathcal{R}$-codimensão de $f$ é finita $e$

$$
\mathcal{O}_{n}=J f_{0}+\mathbb{K}\left\{\dot{f}_{1}, \ldots, \dot{f}_{n}\right\}
$$

\subsection{Singularidades simples}


Proposição 1.35. Seja $f \in \mathcal{M}_{n}^{2}$, isto é, o é uma singularidade. Então $f$ tem $\mathcal{R}_{e}$-codimensão 1 , se e somente se, $f$ tem uma singularidade não degenerada em 0 , ou seja $f$ é Morse. Neste caso $f$ é $\mathcal{R}$-equivalente a um germe da forma

$$
\left(x_{1}, \ldots, x_{n}\right)=-x_{1}^{2}-\ldots-x_{p}^{2}+x_{p+1}^{2}+\ldots+x_{n}^{2} .
$$

Definição 1.36. Seja $f \in \mathcal{M}_{n}^{2}$ tal que a $\mathcal{R}_{e}-\operatorname{codf} \geq 2$ então a matriz Hessiana de $f$ é singular, logo tem rank $r<n$. O inteiro $c=n-r>0$ é chamado de corank de $f$ e é denotado por corankf.

\section{Proposição 1.37. ( Lema da Decomposição ou Splitting Lemma)}

Seja $f \in \mathcal{M}_{n}^{2}$ um germe finitamente determinado e corank c. Então $f$ é $\mathcal{R}$-equivalente a

$$
f\left(x_{1}, \ldots, x_{n}\right)=h\left(x_{1}, \ldots, x_{c}\right) \pm x_{c+1}^{2} \pm \ldots \pm x_{n}^{2} .
$$

onde $h \in \mathcal{M}_{n}^{3}$.

Proposição 1.38. Os germes $f$ e $h$ do Splitting Lemma têm a mesma $\mathcal{R}_{e^{-}}$ codimensão.

Proposição 1.39. Seja $f \in \mathcal{M}_{n}^{2}$ de corank 1 e $\mathcal{R}_{e}$-codimensão $k$. Então

$$
f\left(x_{1}, \ldots, x_{n}\right) \stackrel{\mathcal{R}}{\sim} \pm x_{1}^{k+1} \pm x_{2}^{2} \pm \ldots \pm x_{n}^{2}
$$

Dizemos que $f$ é uma singularidade $A_{k}$.

Definição 1.40. Seja $G$ um grupo de Lie agindo numa variedade $M e$ $x \in M$. Dizemos que uma órbita $G x$ é simples se existe uma vizinhança suficientemente pequena de $g_{0} x$ em $M$, para algum $g_{0} \in G$, que encontra somente um número finito de órbitas.

Proposição 1.41. Se tal vizinhança existe para $g_{0} x$, então existe para $g x, \forall g \in$ $G$. 
Definição 1.42. Um germe $f \in \mathcal{M}_{n}$ é simples se é $k$-determinado para algum $k$ e seu $k$-jato como um elemento de $J^{N}(n, 1), N \geq k$ tem uma vizinhança que encontra somente um número finito de $\mathcal{R}^{N}$-órbitas.

Proposição 1.43. Se $f \in \mathcal{M}_{n}$ é simples, então corank $f \leq 2$.

Assim concluindo, temos o seguinte resultado.

Teorema 1.44. (Arnol'd, [2]) Seja $f \in \mathcal{M}_{n}$. Então $f$ é simples, se somente se, é $\mathcal{R}$-equivalente a um dos seguintes:

$A_{k}: \quad f\left(x, y, x_{3}, \ldots, x_{n}\right)= \pm x^{k+1} \pm y^{2}+Q, \quad \mathcal{R}_{e}-\operatorname{codf}=k, \quad k \geq 1$.

$D_{k}: \quad f\left(x, y, x_{3}, \ldots, x_{n}\right)=x^{2} y \pm y^{k-1}+Q, \quad \mathcal{R}_{e}-\operatorname{codf}=k, \quad k \geq 4$.

$E_{6}: \quad f\left(x, y, x_{3}, \ldots, x_{n}\right)=x^{3} \pm y^{4}+Q, \quad \mathcal{R}_{e}-\operatorname{cod} f=6$.

$E_{7}: \quad f\left(x, y, x_{3}, \ldots, x_{n}\right)=x^{3} \pm x y^{3}+Q, \quad \mathcal{R}_{e}-\operatorname{codf}=7$

$E_{8}: \quad f\left(x, y, x_{3}, \ldots, x_{n}\right)=x^{3} \pm y^{5}+Q, \quad \mathcal{R}_{e}-\operatorname{cod} f=8$.

onde $Q$ é forma quadrática, $Q\left(x_{3}, \ldots, x_{n}\right)= \pm x_{3}^{2} \pm \ldots \pm x_{n}^{2}$.

Nota 1.45. Quando $\mathbb{K}=\mathbb{C}$, cada uma das classses acima tem uma única forma normal, que podemos tomar com todos os sinais dos coeficientes positivos.

Nota 1.46. Os resultados de determinação finita e classificação de singularidades apresentados neste capítulo se verificam também em $\varepsilon_{n}$, anel local de germes $C^{\infty}($ ver [16]).

Nota 1.47. (Princípio de Reconhecimento de Singularidades Simples) 
Um polinômio $f\left(x_{1}, \ldots, x_{n}\right)$ é quasi-homogêneo de grau d se existe uma $n-\operatorname{upla}\left(w_{1}, \ldots, w_{n}\right), w_{i} \in \mathbb{N}^{*}$, tal que $f\left(\lambda^{w_{1}} x_{1}, \lambda^{w_{2}} x_{2}, \ldots, \lambda^{w_{n}} x_{n}\right)=\lambda^{d} f\left(x_{1}, \ldots, x_{n}\right)$, $\forall \lambda \in \mathbb{C}^{*}$.

As formas normais das singularidades simples são polinômios quasi-homogêneos. Com base neste fato, é possível estabelecer o seguinte princípio de reconhecimento: dado um polinômio $f\left(x_{1}, \ldots, x_{n}\right)$ não necessariamente quasihomogêneo, $f$ é equivalente a uma singularidade simples, se existir uma $n-$ upla $w=\left(w_{1}, \ldots, w_{n}\right)$ tal que $f(x)=f_{0}(x)+f_{1}(x)$ em que $f_{0}(x)$ é uma das formas normais quasi-homogêneas do Teorema de Arnol'd 1.44, de tipo we de grau d e o grau de $f_{1}$ é maior do que d.

\subsection{Singularidades não isoladas}

Nesta seção vamos considerar germes de funções $f: \mathbb{C}^{n+1} \rightarrow \mathbb{C}$ com o conjunto crítico suave 1-dimensional. Estas singularidades são chamadas "lines singularities"e foram estudadas por Siersma e seus colaboradores. A principal referência para esta seção é o artigo de A. Zaharia [56].

Vamos denotar as coordenadas em $\left(\mathbb{C}^{n+1}, 0\right)$ por $(x, y)=\left(x, y_{1}, \ldots, y_{n}\right)$. Considere a reta $\mathcal{L}=\left\{y_{1}=\ldots=y_{n}=0\right\}$, seja $I=\left(y_{1}, \ldots, y_{n}\right) \subseteq \mathcal{O}_{n+1}$ e $D_{I}$ o grupo de isomorfismos analíticos locais $\varphi:\left(\mathbb{C}^{n+1}, 0\right) \rightarrow\left(\mathbb{C}^{n+1}, 0\right)$ tais que $\varphi(\mathcal{L})=\mathcal{L}$. Então $D_{I}$ age em $I^{2}$ e para $f \in I^{2}$ o espaço tangente de $f$ com respeito a essa ação é o ideal definido por

$$
\tau(f)=\mathcal{M}\left(\frac{\partial f}{\partial x}\right)+I\left(\frac{\partial f}{\partial y_{1}}, \ldots, \frac{\partial f}{\partial y_{n}}\right)
$$

enquanto a codimensão de $f$ é

$$
C(f)=\operatorname{dim}_{\mathbb{K}} \frac{I^{2}}{\tau(f)} .
$$


Uma singularidade 1-dimensional é um germe $f \in I^{2}$ tal que $C(f)<\infty$. Geometricamente , $f \in I^{2}$ é uma singularidade 1-dimensional, se e somente se, o lugar singular de $f$ é $\mathcal{L}$ e para todo $x \neq 0$ o germe de $f$ em $(x, 0) \in \mathcal{L}$ é equivalente a $y_{1}^{2}+\ldots+y_{n}^{2}$.

Teorema 1.48. Um germe $f \in I^{2}$ é $D_{I^{-}}$-simples, se e somente se, $f$ é $D_{I^{-}}$ equivalente a um dos germes da seguinte tabela:

\begin{tabular}{|c|c|c|c|}
\hline Nome & Forma Normal & Condições & Jato determinado \\
\hline$A_{\infty}$ & $y_{1}^{2}+\ldots+y_{n}^{2}$ & & 2 \\
\hline$D_{\infty}$ & $x y_{1}^{2}+y_{2}^{2} \ldots+y_{n}^{2}$ & & 3 \\
\hline$J_{k, \infty}$ & $x^{k} y_{1}^{2}+y_{1}^{3}+y_{2}^{2} \ldots+y_{n}^{2}$ & $k \geq 2$ & $k+2$ \\
\hline$T_{\infty, K, 2}$ & $x^{2} y_{1}^{2}+y_{1}^{k}+y_{2}^{2} \ldots+y_{n}^{2}$ & $k \geq 4$ & $k$ \\
\hline$Z_{k, \infty}$ & $x y_{1}^{3}+x^{k+2} y_{1}^{2}+y_{2}^{2}+\ldots+y_{n}^{2}$ & $k \geq 1$ & $k+4$ \\
\hline$W_{1, \infty}$ & $x^{3} y_{1}^{2}+y_{1}^{4}+y_{2}^{2}+\ldots+y_{n}^{2}$ & & 5 \\
\hline$T_{\infty, q, r}$ & $x y_{1} y_{2}+y_{1}^{q}+y_{2}^{r}+y_{3}^{2}+\ldots+y_{n}^{2}$ & $q \geq r \geq 3$ & $q$ \\
\hline$Q_{k, \infty}$ & $x^{k} y_{1}^{2}+y_{1}^{3}+x y_{2}^{2}+y_{3}^{2}+\ldots+y_{n}^{2}$ & $k \geq 2$ & $k+2$ \\
\hline$S_{1, \infty}$ & $x^{2} y_{1}^{2}+y_{1}^{2} y^{2}+x y_{2}^{2}+y_{3}^{2}+\ldots+y_{n}^{2}$ & & 4 \\
\hline
\end{tabular}




\section{Capítulo 2}

\section{Estratificação e interseção completa}

Neste capítulo apresentamos resultados básicos da teoria de estratificação de Thom-Whitney e também estudamos propriedades básicas de interseção completa que serão utilizados neste trabalho. As principais referências são: [55], [29], [12] e [26].

\subsection{Conjuntos algébricos, semialgébricos e va- riedades analíticas}

Definição 2.1. Um conjunto $A \subset \mathbb{K}^{n}(\mathbb{K}=\mathbb{R}$ ou $\mathbb{C})$, é algébrico quando existe uma aplicação polinomial $f: \mathbb{K}^{n} \rightarrow \mathbb{K}^{p}, f=\left(f_{1}, \ldots, f_{p}\right)$ tal que, $A=\left\{x \in \mathbb{K}^{n} \mid f_{1}(x)=\ldots=f_{p}(x)=0\right\}$, ou seja $A=f^{-1}(0)$.

Proposição 2.2. (a) Sejam $A$ e $B$ conjuntos algébricos em $\mathbb{K}^{n}$. Então $A \cup B$ e $A \cap B$ são conjuntos algébricos.

(b) Seja $B \subset \mathbb{K}^{m}$ um conjunto algébrico e $f: \mathbb{K}^{n} \rightarrow \mathbb{K}^{m}$ uma aplicação polinomial. Então $f^{-1}(B) \subset \mathbb{K}^{n}$ é um conjunto algébrico. 
A proposição anterior sugere a seguinte pergunta: a imagem de um conjunto algébrico por uma aplicação polinomial é um conjunto algébrico? Isso nem sempre é verdade. Vejamos um exemplo.

Exemplo 2.3. Sejam $\pi: \mathbb{R}^{2} \rightarrow \mathbb{R}$ a projeção $\pi(x, y)=x$ e $S^{1}=\{(x, y) \in$ $\left.\mathbb{R}^{2} \mid x^{2}+y^{2}=1\right\}$. Temos que $S^{1}$ é um conjunto algébrico, mas $\pi\left(S^{1}\right)=[-1,1]$ não o é.

Este exemplo motiva a definição de uma classe mais geral do que a dos conjuntos algébricos, a saber, a classe dos conjuntos semialgébricos.

Definição 2.4. Um conjunto $A \subset \mathbb{R}^{n}$ é semialgébrico básico quando existem funções polinomiais $f, g_{1}, \ldots, g_{k}$, definidas em $\mathbb{R}^{n}$, tais que:

$$
A=\left\{x \in \mathbb{R}^{n} \mid f(x)=0\right\} \cap\left(\cap_{i=1}^{k}\left\{x \in \mathbb{R}^{n} \mid g_{i}(x)>0\right\}\right) .
$$

Definição 2.5. Um conjunto semialgébrico é a reunião finita de conjuntos semialgébricos básicos.

Apresentamos na sequência algumas propriedades dos conjuntos semialgébricos em $\mathbb{R}^{n}$.

Nota 2.6. (1) Os conjuntos algébricos são, claramente, conjuntos semialgébricos.

(2) O conjunto $X=\left\{(x, y) \in \mathbb{R}^{2} \mid x^{2}+y^{2} \leq 1\right\}$ é semialgébrico, mas não é algébrico.

(3) Em $\mathbb{R}$, um conjunto semialgébrico é a reunião de intervalos abertos, fechados, semiabertos e pontos.

(4) Se $A$ e $B$ são conjuntos semialgébricos em $\mathbb{R}^{n}$, então $A \cup B, A \cap B$, $A-B, \mathbb{R}^{n}-A$ e $\mathbb{R}^{n}-B$ são também semialgébricos.

(5) Se $A \subset \mathbb{R}^{n}$ e $B \subset \mathbb{R}^{m}$ são conjuntos semialgébricos, então $A \times B \subset$ $\mathbb{R}^{n} \times \mathbb{R}^{m}$ é um conjunto semialgébrico. 
Proposição 2.7. Se $A \subset \mathbb{R}^{n}$ é um conjunto semialgébrico, então o fecho de $A$, denotado por $\bar{A}$ é um conjunto semialgébrico.

A proposição a seguir decorre de um teorema estrutural para conjuntos semialgébricos (ver Teorema da decomposição cilíndrica [4]).

Proposição 2.8. Se $A \subset \mathbb{R}^{n}$ é um conjunto semialgébrico, então $A$ tem um número finito de componentes conexas e cada uma destas componentes é ainda um conjunto semialgébrico.

Teorema 2.9. (Tarski-Seidenberg [4]) A imagem de um conjunto semialgébrico $A \subset \mathbb{R}^{n}$ por uma projeção $\pi: \mathbb{R}^{n} \rightarrow \mathbb{R}^{n-1}$ é um conjunto semialgébrico.

Corolário 2.10. A imagem de um conjunto semialgébrico por uma aplicação polinomial é um conjunto semialgébrico.

Definição 2.11. Um germe de espaço analítico $(V, 0)$ em torno da origem em $\mathbb{K}^{n}$ é o germe do subconjunto

$$
V=\mathcal{V}\left(f_{1}\right) \cap \ldots \cap \mathcal{V}\left(f_{r}\right)=\mathcal{V}\left(f_{1}, \ldots, f_{r}\right)
$$

onde $f_{1}, \ldots, f_{r} \in \mathcal{O}_{n}$. Definimos o ideal de um germe de espaço analítico $V$ por

$$
\mathcal{I}(V)=\left\{f \in \mathcal{O}_{n} \mid V \subset f^{-1}(0)\right\}
$$

Dizemos que um germe de espaço analítico $V$ é irredutível quando para quaisquer germes analíticos $V_{1}$ e $V_{2}$ tais que $V=V_{1} \cup V_{2}$, então $V=V_{1}$ ou $V=V_{2}$. Neste caso dizemos que $V$ é uma variedade analítica. 


\subsection{Estratificações}

A ideia básica da teoria de estratificação é decompor um espaço singular em variedades regulares, que chamamos de estratos, de tal forma que cada estrato consista de pontos igualmentes "ruins", num certo sentido.

Definição 2.12. Seja $M$ uma variedade suave e $V \subset M$. Uma estratificação localmente finita de $V$ é uma partição de $V$ em subvariedades de $M$ (chamadas de estratos) tais que, para todo ponto de $V$ existe uma vizinhança em $M$ que encontra um número finito de estratos.

Notação: Indicamos por $\left\{V_{\alpha}\right\}$ a estratificação de $V$ com estratos $V_{\alpha}$.

As noções mais importante na teoria de estratificação são as condições de regularidade entre os estratos.

Podemos pensar numa condição de regularidade como sendo um controle de como os estratos de uma estratificação se encontram. Introduziremos dois conceitos principais na teoria de estratificação: a condição de fronteira e as condições de regularidade de Whitney.

Definição 2.13. Uma estratificação $V=V_{1} \cup \ldots \cup V_{p}$ satisfaz a condição de fronteira se, sempre que $V_{i} \cap \overline{V_{j}} \neq \emptyset$, tem se que $V_{i} \subset \overline{V_{j}}$.

Definição 2.14. Uma estratificação $V=V_{1} \cup \ldots \cup V_{p}$ é algébrica, semialgébrica ou analítica, se cada variedade regular $V_{i}$ é um conjunto algébrico, semialgébrico ou analítico respectivamente.

Definição 2.15. A estratificação $\left\{V_{\alpha}\right\}$ satisfaz às condições de Whitney se para todo par $\left(V_{\alpha}, V_{\beta}\right)$ de estratos, tais que $V_{\beta}$ esteja no fecho de $V_{\alpha}$ e para todo ponto y de $V_{\beta}$ temos: 
a) Para toda sequência de pontos $x_{i}$ de $V_{\alpha}$ convergindo para $y$, tal que o limite $\lim _{i \rightarrow \infty} T_{x_{i}}\left(V_{\alpha}\right)$ na Grassmanniana correspondente é $T$, então $T$ contém $T_{y}\left(V_{\beta}\right)$.

b) Se além disso dada qualquer sequência $y_{i}$ de pontos de $V_{\beta}$ com $\lim _{i \rightarrow \infty} y_{i}=$ y e tal que o limite de direções $\lim _{i \rightarrow \infty} \overline{x_{i} y_{i}}$ no espaço projetivo é $\lambda$, então $T$ contém $\lambda$.

Estas são as chamadas condição (a) e condição (b) de Whitney.

Whitney mostrou em [55] que toda variedade analítica complexa admite uma estratificação satisfazendo estas duas condições.

Uma estratificação que satisfaz às condições de Whitney e à condição de fronteira é chamada estratificação de Whitney ou estratificação Whitney regular.

Os Lemas de Isotopia de Thom que apresentamos a seguir são generalizações do Teorema de Morse para variedades.

Definição 2.16. (i) Seja $A$ um subconjunto de uma variedade suave $N$, $\left\{\mathcal{A}_{\alpha}\right\}$ uma estratificação de $A$, e $f$ uma aplicação suave de $N$ em uma outra variedade suave $P$. Dizemos que o conjunto estratificado $(A, \mathcal{A})$ é topologicamente trivial sobre $P$ se existem um conjunto estratificado $(F, \mathcal{F})$ e um homeomorfismo $h: A \approx P \times F$ com $f=\pi_{P} \circ h$, levando $\mathcal{A}$ no produto estratificado $P \times \mathcal{F}\left(\pi_{P}\right.$ denota a projeção de $\left.P\right)$.

(ii) Dizemos que $(A, \mathcal{A})$ é localmente trivial sobre $P$ se cada ponto $y \in P$ tem uma vizinhança aberta $V$ tal que $\left(A \cap f^{-1}(V), \mathcal{A} \cap f^{-1}(V)\right)$ é trivial sobre $V$.

Teorema 2.17. (Primeiro Lema de Isotopia de Thom [29]) Sejam A um subconjunto localmente fechado de uma variedade suave $N$ e $\mathcal{A}$ uma estrati- 
ficação de Whitney de A. Seja $f: N \rightarrow P$ uma aplicação suave tal que para cada estrato $X \in \mathcal{A},\left.f\right|_{X}$ é uma submersão e $\left.f\right|_{\bar{X} \cap A}$ é uma aplicação própria. Então $(A, \mathcal{A})$ é localmente trivial sobre $P$.

Suponhamos que $M, M^{\prime}$ e $P$ sejam variedades suaves e que $f: M \rightarrow P$ seja $\mathcal{C}^{\infty}$. Suponha também que $S \subset M$ e $S^{\prime} \subset M^{\prime}$ sejam variedades Whitney estratificadas. Além disso, seja $g: M^{\prime} \rightarrow M$ uma aplicação suave tal que $g\left(S^{\prime}\right) \subset S$.

Definição 2.18. Sejam $X$ e $Y$ subvariedades regulares de $M^{\prime}$, e seja $p \in$ $Y$. Suponha que $\left.g\right|_{X}$ e $\left.g\right|_{Y}$ tem posto constante. Dizemos que o par $(X, Y)$ satisfaz à condição $\left(a_{g}\right)$ de Thom em $p$, se vale o seguinte: seja $\left\{m_{i}\right\}$ uma sequência de pontos em $X$ tal que $m_{i} \rightarrow p$. Suponha que a sequência de planos $\operatorname{ker}\left(\left.d g\right|_{X}\left(m_{i}\right)\right) \subset T_{m_{i}} M^{\prime}$ convirja para um plano $\tau$. Então $k e r\left(\left.d g\right|_{Y}(p)\right) \subset \tau$.

Definição 2.19. Dizemos que o par $(X, Y)$ satisfaz a condição $\left(a_{g}\right)$ de Thom se ele satisfaz a condição $\left(a_{g}\right)$ para todo $p \in Y$.

Definição 2.20. Dizemos que g é uma aplicação de Thom ( em p) se as seguintes condições são satisfeitas:

(a) $\left.g\right|_{S^{\prime}}$ e $\left.f\right|_{S}$ são próprias.

(b) para cada estrato $X$ de $S,\left.f\right|_{X}$ é uma submersão.

(c) para cada estrato $X^{\prime}$ de $S^{\prime}, g\left(X^{\prime}\right) \subset X$ e $g: X^{\prime} \rightarrow X$ é uma submersão (logo $\left.g\right|_{X^{\prime}}$ tem posto constante).

(d) qualquer par $\left(X^{\prime}, Y^{\prime}\right)$ de $S^{\prime}$ satisfaz a condição $\left(a_{g}\right)$ de Thom (a qual faz sentido devido ao item $(c))$. 
Teorema 2.21. (Segundo Lema de Isotopia de Thom [29]) Se g é uma aplicação de Thom sobre $P$, então g é localmente trivial sobre $P$.

\subsection{Interseção completa}

Definição 2.22. Um germe de um conjunto analítico complexo $(X, 0) n a$ origem $0 \in \mathbb{C}^{n}$ é chamado uma singularidade de interseção completa se todas as componentes irredutiveis de $(X, 0)$ têm a mesma dimensão, digamos $m$, e $(X, 0)$ pode ser definido como o conjunto dos zeros de $n-m$ funções analiticas.

Isto significa que em uma pequena bola aberta $B_{\epsilon}$ centrada na origem de $\mathbb{C}^{n}$ tem-se:

$$
X \cap B_{\epsilon}=\left\{x \in B_{\epsilon} \mid f_{1}(x)=\ldots=f_{n-m}(x)=0\right\}
$$

$\operatorname{para} f_{1}, \ldots, f_{n-m} \in \mathcal{O}_{n}$

Definição 2.23. Uma interseção completa $(X, 0)$ é uma ICIS (interseção completa com singularidade isolada) quando existe uma vizinhança $U$ de 0 tal que Sing $(X) \cap U=\{0\}$ em que Sing $(X)$ é o conjunto singular de $X$.

O seguinte resultado pode ser encontrado em [16].

Proposição 2.24. Seja $f:\left(\mathbb{C}^{n}, 0\right) \rightarrow\left(\mathbb{C}^{p}, 0\right), n \geq p$. As seguintes condições são equivalentes:

(1) $f$ é $\mathcal{K}$-finitamente determinado

(2) $f^{-1}(0) \cap \operatorname{Sing}(f)=\{0\}$

(3) $X=f^{-1}(0)$ é uma ICIS

(4) Existe $l$ tal que $J(f)+<f_{1}, \ldots, f_{p}>\supseteq \mathcal{M}_{n}^{l}$, em que $J(f)$ é o ideal 
jacobiano de $f$, isto é, o ideal gerado pelos menores $p \times p$ da matriz jacobiana de $f$. 


\section{Capítulo 3}

\section{Singularidades no infinito}

O estudo das fibrações associadas a funções polinomiais $\mathbb{C}^{n} \rightarrow \mathbb{C}$ foi introduzido por Broughton [9], que exibiu o seguinte exemplo de um polinômio não singular cujo tipo topológico das fibras não é constante.

Exemplo 3.1. Considere $f: \mathbb{C}^{2} \rightarrow \mathbb{C}, f(x, y)=x+x^{2} y$.

É fácil verificar que $f$ não tem pontos singulares. Temos que $f^{-1}(\epsilon)=\{y=$ $\left.(\epsilon-x) / x^{2}\right\}$ para $\epsilon \neq 0$ e $f^{-1}(0)=\{x(x y+1)=0\}$. Portanto $f^{-1}(\epsilon)$ é homeomorfo a $\mathbb{C}^{*}=\mathbb{C} \backslash\{0\}$, enquanto $f^{-1}(0)$ é homeomorfo à união disjunta $\mathbb{C} \cup \mathbb{C}^{*}$, logo $f$ não é uma fibração em qualquer vizinhança da fibra $f^{-1}(0)$.

Enquanto que no estudo local das singularidades, a presença de uma singularidade é a razão para a não trivialidade da fibração local associada a um germe singular, o exemplo acima mostra que no contexto global, as fibras de uma aplicação polinomial $f$, próximas a uma fibra $f^{-1}\left(t_{0}\right)$ podem ser topologicamente distintas de $f^{-1}\left(t_{0}\right)$, mesmo sem a presença de singularidades.

Desta forma questões naturais no estudo global de singularidades definidas por polinômios incluem a caracterizações destes valores (valores atípicos) e a classificação das singularidades associadas a tais valores. 
Neste capítulo, nosso objetivo é estudar as singularidades no infinito. Começamos com algumas definições e resultados para determinar tais singularidades e posteriormente estudaremos os valores atípicos.

As principais referências para este capítulo são [32], [45], [46], [47] e [56].

$\mathrm{Na}$ sequência, indicaremos por $\mathbb{K}\left[y_{1}, \ldots, y_{n}\right]$ o anel dos polinômios com coeficientes em $\mathbb{K}=\mathbb{R}$ ou $\mathbb{C}$ nas variáveis $y_{1}, \ldots, y_{n}$. Nos capítulos seguintes, nosso principal interesse será no estudo dos polinômios complexos. Por esta razão, nesta seção vamos em geral considerar polinômios sobre $\mathbb{C}$. Alguns resultados são válidos também para polinômios reais.

Na próxima seção vamos recordar alguns conceitos do estudo das singularidades locais para posteriormente concentrar nosso estudo globalmente.

\subsection{Germes de funções com singularidade iso- lada}

Seja $g:\left(\mathbb{K}^{n}, 0\right) \rightarrow(\mathbb{K}, 0)$, para $n \geq 2$ um germe de função analítica com singularidade isolada. Isto significa que o ideal Jacobiano no anel dos germes na origem das funções analíticas reais ou complexas

$$
J(g)=\left(\frac{\partial g}{\partial x_{1}}, \ldots, \frac{\partial g}{\partial x_{n}}\right)
$$

é de codimensão finita, equivalentemente que o lugar dos zeros $Z(J(g))$ é localmente $\{0\}$. Se o lugar dos zeros é localmente vazio dizemos que $g$ é não singular na origem.

Boa parte da literatura referente a singularidades é voltada para o estudo de invariantes de aplicações e funções singulares. Um dos invariantes analiticos de $g$ é o número de Milnor [30] 


$$
\mu(g)=\operatorname{dim}_{\mathbb{C}} \frac{\mathbb{C}\left\{x_{1}, \ldots, x_{n}\right\}}{J(g)}
$$

O número de Milnor depende do conjunto dos zeros de $g$, que denotamos por $Z(g)$, o qual é uma hipersuperfície, mais precisamente se trocarmos $g$ por $u g$ onde $u$ é um germe analítico e $u(0) \neq 0$, então $Z(g)=Z(u g)$ e $\mu(g)=\mu(u g)$ Também se associa a um germe $g$ a sua fibração local, chamada fibração de Milnor. Então $\mu(g)$ pode ser interpretado como o rank do (n-1)-ésimo grupo de homologia da fibra de Milnor, consequentemente é um invariante topológico.

A seguir voltaremos nossa atenção para as ferramentas e técnicas do estudo global das singularidades.

\subsection{Singularidades no infinito}

Definição 3.2. Seja $f: \mathbb{C}^{n} \rightarrow \mathbb{C}$. Dizemos que $f$ é topologicamente trivial em $t_{0} \in \mathbb{C}$ se existe uma vizinhança $D$ de $t_{0} \in \mathbb{C}$ tal que a restrição $f \mid$ : $f^{-1}(D) \rightarrow D$ é uma fibração topologicamente trivial. Se $t_{0}$ não satisfaz essa propriedade, então dizemos que $t_{0}$ é um valor atípico e que $f^{-1}\left(t_{0}\right)$ é uma fibra atípica. Denotamos por Atyp $(f)$ o conjunto dos valores atipicos de $f$.

No Exemplo 3.1 o conjunto Atyp $(f)$ consiste somente do valor 0. Em geral, a seguinte relação se verifica

$$
f(\operatorname{Sing}(f)) \subset \operatorname{Atyp}(f),
$$

onde $\operatorname{Sing}(f)=Z(J(f))$ denota o lugar singular de $f$.

Como $\operatorname{Sing}(f)$ é um conjunto algébrico e $f: \mathbb{C}^{n} \rightarrow \mathbb{C}$ é uma função algébrica, 
então $f(\operatorname{Sing}(f))$ é um conjunto finito. O conjunto dos valores atípicos Atyp $(f)$ também é finito, a prova de que o conjunto dos valores atípicos Atyp $(f)$ é finito pode ser encontrada em [12], pg 20 Prop. 4.1. O conjunto $\mathbb{C} \backslash \operatorname{Atyp}(f)$ é portanto um conjunto conexo. Assim, $\forall t_{1}$ e $t_{2} \in \mathbb{C} \backslash \operatorname{Atyp}(f)$, as fibras $f^{-1}\left(t_{1}\right)$ e $f^{-1}\left(t_{2}\right)$ são homeomorfas. Portanto podemos denominar de "fibra geral" a qualquer $f^{-1}(t), t \in \mathbb{C} \backslash \operatorname{Atyp}(f)$.

Uma evidência para a importância do estudo das singularidades no infinito é o seguinte problema.

Conjectura 3.3. (Conjectura Jacobiana) $S$ e $f: \mathbb{C}^{2} \rightarrow \mathbb{C}$ não tem pontos críticos mas tem singularidades no infinito então, para qualquer polinômio $h: \mathbb{C}^{2} \rightarrow \mathbb{C}$, o conjunto singular de $(f, h): \mathbb{C}^{2} \rightarrow \mathbb{C}^{2}$ é não vazio.

\subsubsection{Como encontrar valores atípicos}

Seja $f: \mathbb{C}^{n} \rightarrow \mathbb{C}$ um polinômio de grau $d, f=f_{1}+f_{2}+\ldots+f_{d-1}+f_{d}$, em que $f_{k}$ é um polinômio homogêneo de grau $k, k=1, \ldots, d$. Seja $\tilde{f}\left(x, x_{0}\right)$ a homogenização de $f$ pela nova variável $x_{0}$. Consideremos o fecho em $\mathbb{P}^{n} \times \mathbb{C}$ do gráfico de $f$, isto é, a hipersuperfície

$$
\mathbb{X}=\left\{\left(\left(x ; x_{0}\right), t\right) \in \mathbb{P}^{n} \times \mathbb{C} \mid F\left(x, x_{0}, t\right)=\tilde{f}\left(x, x_{0}\right)-t x_{0}^{d}=0\right\}
$$

Para cada $t \in \mathbb{C}$ fixado, indicaremos por $X_{t}$ a fibra $f^{-1}(t)$ em $\mathbb{C}^{n}$ e seja

$$
\bar{X}_{t}=\left\{\left(x ; x_{0}\right) \in \mathbb{P}^{n} \mid F\left(x, x_{0}, t\right)=0\right\}
$$

seu fecho projetivo em $\mathbb{P}^{n}$. Seja

$$
\tau: \mathbb{X} \rightarrow \mathbb{C}, \quad \tau\left(\left(x: x_{0}\right), t\right)=t
$$


a projeção em $\mathbb{C}$ e vamos denotar por $H^{\infty}$ o hiperplano no infinito $\left\{x_{0}=\right.$ $0\} \subset \mathbb{P}^{n}$. Seja $\mathbb{X}^{\infty}=\mathbb{X} \cap\left(H^{\infty} \times \mathbb{C}\right)$ a parte no infinito de $\mathbb{X}$. Note que $\tau$ é uma aplicação própria, enquanto $f$ não é própria.

Verifica-se que as singularidades no infinito de $\mathbb{X}$ estão contidas em $\mathbb{X}^{\infty}$, a saber $\mathbb{X}_{\text {Sing }}=\Sigma^{\infty} \times \mathbb{C}$, onde:

$$
\Sigma^{\infty}=\left\{\frac{\partial f_{d}}{\partial x_{1}}=\ldots=\frac{\partial f_{d}}{\partial x_{n}}=0, f_{d-1}=0\right\} \subset H^{\infty} .
$$

As singularidades de $f$, isto é, o conjunto afim $\operatorname{Sing}(f)=Z\left(\frac{\partial f}{\partial x_{1}}, \ldots, \frac{\partial f}{\partial x_{n}}\right)$ pode ser identificado com as singularidades de $\tau$ em $\mathbb{X} \backslash \mathbb{X}^{\infty}$.

Quando $f$ tem apenas singularidades isoladas, a álgebra quociente :

$$
\mathbb{C}\left[x_{1}, \ldots, x_{n}\right] / J(f)
$$

é um espaço vetorial complexo de dimensão finita e essa dimensão $\mu(f)$ é chamada número de Milnor global. Pode-se provar que $\mu(f)$ é a soma total dos números de Milnor locais de $f$ nesses pontos singulares [48].

A seguir vamos descrever o comportamento de $f$ no infinito nos casos em que $\Sigma^{\infty}=\emptyset$ ou quando $\operatorname{dim} \Sigma^{\infty}=0$.

$1^{\mathbf{o}}$ caso: Quando $\Sigma^{\infty}=\emptyset, f$ tem apenas singularidades isoladas. De fato, se $\operatorname{Sing}(f)$ é não isolado, obtemos que $\overline{\operatorname{Sing}(f)} \cap H^{\infty} \neq \emptyset$. Por outro lado temos as seguintes condições: $\operatorname{Sing}(F) \cap H^{\infty}=\Sigma^{\infty}$ e $\overline{\operatorname{Sing}(f)} \cap H^{\infty} \subset$ $\operatorname{Sing}(F) \cap H^{\infty}=\Sigma^{\infty}$, logo segue o resultado. As singularidades da restrição de $\tau$ a $\mathbb{X} \backslash \mathbb{X}^{\infty}$ são exatamente as singularidades de $f$. Além disso, a restrição de $\tau$ a $\mathbb{X}^{\infty}$ é uma submersão e $\tau$ é também uma submersão para $\mathcal{N} \cap\left(\mathbb{X} \backslash \mathbb{X}^{\infty}\right)$ para alguma vizinhança $\mathcal{N}$ de $\mathbb{X}^{\infty}$.

Aplicando agora o Teorema de Ehresmann [14] para a variedade $\mathcal{N} \cap\left(\mathbb{X} \backslash \mathbb{X}^{\infty}\right)$ 
com a fronteira $\mathcal{N} \cap \mathbb{X}^{\infty}$, e para a aplicação própria submersiva $\tau$, concluímos que $\tau$ é uma fibração localmente trivial $\tau:\left(\mathcal{N} \cap\left(\mathbb{X} \backslash \mathbb{X}^{\infty}\right), \mathbb{X}^{\infty}\right) \rightarrow \mathbb{C}$.

Definição 3.4. Dizemos que $f$ é topologicamente trivial no infinito no valor $t_{0} \in \mathbb{C}$ se existe um conjunto compacto $K \subset \mathbb{C}^{n}$ e um disco $D_{\delta}$ centrado em $t_{0}$ tal que a restrição

$$
f \mid\left(\mathbb{C}^{n} \backslash K\right) \cap f^{-1}\left(D_{\delta}\right) \rightarrow D_{\delta}
$$

é uma fibração topologicamente trivial.

Neste caso, isto é, quando $\Sigma^{\infty}=\emptyset$, segue da discusão acima que não pode haver singularidades no infinito, mais precisamente que o polinômio $f$ é topologicamente trivial no infinito em qualquer valor $t \in \mathbb{C}$. Isso, claro, implica que Atyp $(f)=\operatorname{Sing}(f)$.

$\mathbf{2}^{\mathbf{o}}$ caso: Quando $\operatorname{dim} \Sigma^{\infty}=0$. Observe primeiro que qualquer polinômio em duas variáveis com $\Sigma^{\infty} \neq \emptyset$, verifica a condição $\operatorname{dim} \Sigma^{\infty}=0$. Vamos supor que $f$ tem apenas singularidades isoladas em $\mathbb{C}^{n}$, isto é, $\operatorname{dim}(\operatorname{Sing}(f))=0$.

Aqui precisamos substituir o Teorema de Ehresmann [14] pelo Teorema de Isotopia de Thom [29]. Este é indicado para espaços com estratificação de Whitney. Aqui trabalhamos com a estratificação de Whitney $\mathcal{W}$ de $\mathbb{X}$ tendo $\left(\mathbb{X} \backslash \mathbb{X}^{\infty}\right) \simeq \mathbb{K}^{n}$ como um dos estratos, e os outros estratos são de dimensões menores e mergulhados em $\mathbb{X}^{\infty}$, como segue:

$$
\left(\mathbb{X} \backslash \mathbb{X}^{\infty}\right), \mathbb{X}^{\infty} \backslash\left(\Sigma^{\infty} \times \mathbb{K}\right),\left(\Sigma^{\infty} \times \mathbb{K}\right) \backslash B \text { e } B
$$

onde $B$ é um conjunto finito de pontos.

A aplicação $\tau$ é própria e submersiva em cada estrato de $\mathcal{W}$, exceto no estrato $B$. Tais pontos em que a transversalidade falha, são chamados $\mathcal{W}$ singularidades. Esse conjunto de pontos é denotado por $\operatorname{Sing}^{\infty} f$. Esses 
pontos estão em um número finito de fibras de $\tau$ e denotamos por $A_{\text {inf }}$ os valores correspondente de $\tau$. Com exceção desses valores e dos valores críticos de $f$, os outros valores são não atípicos, e numa vizinhança destes pontos temos uma fibração localmente trivial.

Exemplo 3.5. Seja $f: \mathbb{C}^{3} \rightarrow \mathbb{C}, f(x, y, z)=x+x^{2} y$. O conjunto $\Sigma^{\infty}$ é de dimensão 1. O conjunto de $\mathcal{W}$-singularidades pode ser no máximo de dimensão 1, e é exatamente de dimensão 1. De fato, se fosse de dimensão 0, então poderíamos aplicar o Teorema 3.6(abaixo) para mostrar que a fibra genérica de $f$ é um bouquet de esferas $S^{2}$, mas observe que a fibra especial é $\mathbb{C}^{*} \times \mathbb{C}$, que é homotópica a $S^{1}$.

O próximo teorema é a versão global [48] do Teorema do Bouquet local de J. Milnor [30].

Teorema 3.6. Seja $f: \mathbb{C}^{n} \rightarrow \mathbb{C}$ um polinômio com $\mathcal{W}$-singularidades isoladas no infinito. Então a fibra geral de $f$ tem o tipo de homotopia de um bouquet de esferas de dimensão real $n-1$.

Nota 3.7. Como no caso local, se $f$ tem $\mathcal{W}$-singularidades não-isoladas no infinito, então não podemos esperar que a fibra tenha o tipo de homotopia de um bouquet de esferas como no teorema acima. O Exemplo 3.5 mostra que mesmo quando o polinômio tem fibras suaves ( mais algumas $\mathcal{W}$ singularidades não-isoladas no infinito) o resultado acima não é verdadeiro.

Definição 3.8. Denotamos por $\lambda_{a}$ o número de esferas na fibra de Milnor do germe $t:(\mathbb{X}, a) \rightarrow(\mathbb{K}, b)$ e chamamos o número de Milnor no infinito de $\lambda_{a}$.

Observamos que os números $\lambda_{a}$ dependem da imersão de $\mathbb{C}^{n}$ em $\mathbb{P}^{n}$, em outras palavras, da escolha das coordenadas de $\mathbb{C}^{n}$ a menos de automorfismo. 
Corolário 3.9. Seja $f$ um polinômio com $\mathcal{W}$-singularidades isoladas no infinito. Então:

a) O número $\gamma$ de esferas na fibra geral de $f$ é igual a soma $\mu_{f}+\lambda_{f}$, onde $\mu_{f}$ é o número de Milnor total de $f$ e $\lambda_{f}$ é a soma dos números de Milnor no infinito $\lambda_{a_{i}}$.

Em particular $\gamma$ e $\lambda_{f}$ são invariantes sob difeomorfismos de $\mathbb{C}^{n}$.

b) Denote $\mu_{F_{b}}$ a soma dos números de Milnor de todas as singularidades da fibra $F_{b}$ e $\lambda_{F_{b}}$ a soma de todos os números de Milnor no infinito de $\mathbb{X}_{b} \cap \mathbb{X}^{\infty}$. Então

$$
\mathcal{X}\left(F_{u}\right)-\mathcal{X}\left(F_{b}\right)=(-1)^{n-1}\left(\lambda_{F_{b}}+\mu_{F_{b}}\right) .
$$

onde $F_{u}$ é fibra geral de $f$. 


\section{Capítulo 4}

\section{Classificação de polinômios de grau 3 em $\mathbb{C}^{3}$}

\subsection{Introdução}

Quando estudamos uma família $f\left(x_{1}, \ldots, x_{n}\right)=t$, queremos saber, por exemplo, quais são os pontos singulares no infinito, o tipo das singularidades no infinito, o tipo topológico da fibra genérica, o conjunto de valores de bifurcação, mudanças ocorridas na topologia de uma fibra próxima a um valor de bifurcação, a monodromia no infinito.

Para algumas classes de polinômios, mudanças na topologia dependem somente dos pontos singulares afins e de seus tipos de singularidades. Mas em geral outros efeitos podem produzir mudanças na topologia da fibra, como vimos no Exemplo 3.1 no capítulo 3.

A classificação global das singularidades de aplicações polinomiais é um problema difícil e poucos são os métodos conhecidos. Nesta tese, dois artigos foram de grande importância para o estudo de singularidades no infinito de polinômios de grau 3 em $\mathbb{C}^{3}$. No artigo [11], Bruce e Wall estudam as singu- 
laridades no infinito de aplicações polinomiais através das singularidades das homogenizações destas aplicações definidas no espaço projetivo. Este método será estudado no próximo capítulo.

Neste capítulo, com base no artigo [38], de Siersma e Smeltink, estudamos polinômios de grau $3 \mathrm{em} \mathbb{C}^{3}$. O objetivo é classificar as singularidades no infinito, isoladas e não isoladas que são simples, conforme o Teorema $1.44 \mathrm{e}$ o Teorema 1.48. Como aplicação dos resultados, estudamos a regularidade no infinito de famílias $f\left(x_{1}, \ldots, x_{n}\right)=t$.

\subsection{Cúbicas em $\mathbb{P}^{2}$}

A classificação das formas cúbicas em $\mathbb{P}^{2}$ é um tema clássico que retomamos nesta seção com base na referência [7].

\subsubsection{Forma cúbica não singular em $\mathbb{P}^{2}$}

Dizemos que duas formas cúbicas $f$ e $f^{\prime}$ são linearmente equivalentes se existir um isomorfismo $\theta: \mathbb{C}^{3} \rightarrow \mathbb{C}^{3}$ tal que $f=f^{\prime} \circ \theta$. Notação: $f \cong f^{\prime}$.

Definição 4.1. Seja $f$ uma forma cúbica não singular. Então existem $x_{0}, x_{1}$ $e x_{2} \in \mathbb{C}$ linearmente independentes e $\lambda$ diferente de zero tais que $f \cong$ $x_{0}^{3}+x_{1}^{3}+x_{2}^{3}-3 \lambda x_{0} x_{1} x_{2}, \lambda^{3} \neq-1$.

Definição 4.2. Definimos o $j$ - invariante de $f$ como

$$
j(f)=\frac{\lambda^{3}\left(\lambda^{3}+8\right)^{3}}{\left(\lambda^{3}-1\right)^{3}}
$$

em que $\lambda$ é definido como na definição anterior. 
Lema 4.3. Duas formas cúbicas não singulares $f_{\lambda}$ e $f_{\mu}$ são linearmente equivalentes, se e somente se, $j\left(f_{\lambda}\right)=j\left(f_{\mu}\right)$.

Demonstração. A demonstração pode ser encontrada em [7]

\subsubsection{Formas cúbicas singulares em $\mathbb{P}^{2}$}

Nesta subseção descrevemos as formas normais das cúbicas singulares em $\mathbb{P}^{2}$. Vamos dividir a classificação em duas partes:

(I) Formas cúbicas redutíveis.

(II) Formas cúbicas irredutíveis.

Primeiro consideramos as formas cúbicas redutíveis $f$, diferentes de zero.

Seja portanto $f=l g$, onde $g r(l)=1$ e $g r(g)=2$. Se $g$ é redutível, então $f=l_{1} l_{2} l_{3}$, onde $\operatorname{gr}\left(l_{i}\right)=1$. Dependendo do número de retas distintas e dos pontos de interseção, podemos verificar que existe uma base $\left\{e_{0}, e_{1}, e_{2}\right\}$ de $\mathbb{C}^{3}$ tal que $f\left(x_{0} e_{0}+x_{1} e_{1}+x_{2} e_{2}\right)$ é uma das seguintes formas polinomiais:

(a) $x_{0}^{3}$

(b) $x_{0}^{2} x_{1}$

(c) $x_{0}^{3}+x_{1}^{3}$

(d) $x_{0} x_{1} x_{2}$

Nesses casos chamamos as cúbicas respectivamente de reta tripla, reta dupla mais reta simples, três retas concorrentes e triângulo. Os pontos singulares dessas curvas são os pontos de interseção entre as retas. Portanto 
uma reta tripla e uma reta dupla mais uma reta simples têm infinitos pontos singulares, entretanto três retas concorrentes têm um único ponto singular e um triângulo tem três pontos singulares.

Agora se $g$ é irredutível, podemos encontrar uma base tal que $f\left(x_{0} e_{0}+\right.$ $\left.x_{1} e_{1}+x_{2} e_{2}\right)$ é igual a uma das seguintes formas:

(e) $\left(x_{0}^{2}+x_{1} x_{2}\right) x_{1}$

(f) $\left(x_{2}^{2}-x_{0} x_{1}\right) x_{2}$

No caso (e) a reta $\left\{x_{1}=0\right\}$ é tangente a $x_{0}^{2}+x_{1} x_{2}=0$ e no caso (f) a reta $\left\{x_{2}=0\right\}$ tem dois pontos de interseção distintos com $x_{2}^{2}-x_{0} x_{1}=0$. As curvas associadas são chamadas respectivamente de cônica mais tangente e cônica mais corda. Os pontos singulares dessas curvas são os pontos de interseção entre a cônica e a reta, logo a cônica mais tangente tem um ponto singular e a cônica mais corda tem dois pontos singulares.

Agora vamos classificar as formas cúbicas singulares irredutíveis.

Lema 4.4. Suponha que $f$ é uma forma cúbica singular irredutível não nula. Então $\{f=0\}$ tem um único ponto singular e a multiplicidade de $\{f=0\}$ nesse ponto é igual a 2.

Demonstração. A demonstração pode ser encontrada em [15].

Lema 4.5. Suponha que $f$ é uma forma cúbica singular irredutível não nula com duas tangentes simples no ponto singular. Então existe uma base $\left\{e_{0}, e_{1}, e_{2}\right\}$ tal que $f\left(x_{0} e_{0}+x_{1} e_{1}+x_{2} e_{2}\right)=x_{0}^{3}+x_{1}^{3}-x_{0} x_{1} x_{2}$.

Demonstração. A demonstração pode ser encontrada em [7]. 
Definição 4.6. Se $f$ é uma forma cúbica singular irredutível não nula com duas tangentes simples no ponto singular, dizemos que a curva cúbica $\{f=0\}$ é nodal.

Lema 4.7. Suponha que $f$ é uma forma cúbica singular irredutível não nula com uma tangente dupla no ponto singular. Então existe uma base $\left\{e_{0}, e_{1}, e_{2}\right\}$ tal que $f\left(x_{0} e_{0}+x_{1} e_{1}+x_{2} e_{2}\right)=x_{2}^{3}-x_{0} x_{1}^{2}$.

Demonstração. A demonstração pode ser encontrada em [7].

Definição 4.8. Se $f$ é uma forma cúbica singular irredutível não nula com uma tangente dupla no ponto singular, dizemos que $\{f=0\}$ é cuspidal.

Teorema 4.9. (Classificação das cúbicas singulares [15]) Temos oito tipos diferentes de formas cúbicas singulares não nulas, sendo seis delas redutiveis e duas irredutíveis.

(a) reta tripla: $x_{0}^{3}$

(b) reta dupla mais reta simples: $x_{0}^{2} x_{1}$

(c) três retas concorrentes: $x_{0}^{3}+x_{1}^{3}$

(d) triângulo: $x_{0} x_{1} x_{2}$

(e) cônica mais tangente: $\left(x_{0}^{2}+x_{1} x_{2}\right) x_{1}$

(f) cônica mais corda: $\left(x_{2}^{2}-x_{0} x_{1}\right) x_{2}$

(g) nodal: $x_{0}^{3}+x_{1}^{3}-x_{0} x_{1} x_{2}$

(h) cúspide: $x_{2}^{3}-x_{0} x_{1}^{2}$ 


\subsubsection{Classificação de polinômios de grau $3 \mathrm{em} \mathbb{P}^{3}$}

O objetivo desta seção é classificar os polinômios $f: \mathbb{C}^{3} \rightarrow \mathbb{C}, \operatorname{gr} f=3$ no infinito, em que

$$
f=f_{1}\left(x_{0}, x_{1}, x_{2}\right)+f_{2}\left(x_{0}, x_{1}, x_{2}\right)+f_{3}\left(x_{0}, x_{1}, x_{2}\right),
$$

com $f_{i}$ homogêneo de grau $i$, para $i=1,2,3$.

Então seja

$$
f=f_{1}\left(x_{0}, x_{1}, x_{2}\right)+f_{2}\left(x_{0}, x_{1}, x_{2}\right)+f_{3}\left(x_{0}, x_{1}, x_{2}\right),
$$

onde fixamos $f_{3}$ sendo uma forma normal de cúbica de $\mathbb{P}^{2}$ e $F$ a homogenização de $f$ dada por

$$
F=x_{3}^{2} f_{1}\left(x_{0}, x_{1}, x_{2}\right)+x_{3} f_{2}\left(x_{0}, x_{1}, x_{2}\right)+f_{3}\left(x_{0}, x_{1}, x_{2}\right) .
$$

Primeiro vamos analisar quais são os tipos de singularidades de $F$ no infinito. Vamos também estudar a topologia da fibra especial de $F$, isto é, o comportamento topológico no infinito da fibra $\{F=0\}$.

Analisamos simultaneamente os dois casos:

(1) $\operatorname{Sing}(f)=\emptyset$, isto é, sem singularidades na parte afim.

(2) Sing $(f) \neq \emptyset$.

Por uma mudança de coordenadas lineares, podemos assumir que $f_{3}$ é uma forma normal como no Teorema 4.9. Tomemos $f_{1}\left(x_{0}, x_{1}, x_{2}\right)=a_{0} x_{0}+$ $a_{1} x_{1}+a_{2} x_{2}$ e $f_{2}\left(x_{0}, x_{1}, x_{2}\right)=a_{3} x_{0}^{2}+a_{4} x_{0} x_{1}+a_{5} x_{0} x_{2}+a_{6} x_{1}^{2}+a_{7} x_{1} x_{2}+a_{8} x_{2}^{2}$. Então

$F=x_{3}^{2}\left(a_{0} x_{0}+a_{1} x_{1}+a_{2} x_{2}\right)+x_{3}\left(a_{3} x_{0}^{2}+a_{4} x_{0} x_{1}+a_{5} x_{0} x_{2}+a_{6} x_{1}^{2}+a_{7} x_{1} x_{2}+\right.$ 
$\left.a_{8} x_{2}^{2}\right)+f_{3}\left(x_{0}, x_{1}, x_{2}\right)$, onde $f_{3}$ é uma forma normal, como no Teorema 4.9 .

Seja $V=F^{-1}(0) \subset \mathbb{P}^{3}$ a superfície cúbica definida por $F$. Se $P$ é um ponto singular de $V$, podemos escolher coordenadas homogêneas $\left(x_{0}: x_{1}: x_{2}: x_{3}\right)$ com $P$ em (0: 0: 0: 1). Obtemos as coordenadas locais afim em $P$ tomando $x_{3}=1$.

Nota 4.10. Dizemos que $f$ é isomorfa a $g(\cong)$ se a menos de uma transformação linear afim elas são iguais. Usamos transformações tanto na fonte como na meta. Tais transformações podem ser exemplificadas pelas translações $\mathbb{T}_{\alpha, \beta}$ :

$$
\begin{aligned}
& X_{0}=x_{0}-\alpha \\
& X_{1}=x_{1}-\beta \\
& X_{2}=x_{2}-\gamma
\end{aligned}
$$

para a fonte e similar para a meta.

\subsection{Classificação projetiva de $F$}

Vamos classificar $F$, fixando em cada caso uma forma normal para $f_{3}$.

\subsection{1 $f_{3}$ nodal}

Lema 4.11. Por uma mudança de coordenadas lineares podemos supor que $f_{3}=x_{0}^{3}+x_{1}^{3}-x_{0} x_{1} x_{2}$. A menos de translação, $f$ é equivalente a $f=a_{2} x_{2}+a_{3} x_{0}^{2}+a_{4} x_{0} x_{1}+a_{5} x_{0} x_{2}+a_{6} x_{1}^{2}+a_{7} x_{1} x_{2}+a_{8} x_{2}^{2}+x_{0}^{3}+x_{1}^{3}-x_{0} x_{1} x_{2}$. Temos as seguintes situações:

1) Se $a_{8} \neq 0$, então $f$ é regular no infinito. 
2) Se $a_{8}=0, Q=(0: 0: 1: 0)$ é o único ponto singular no infinito e além disso:

a) $a_{2} \neq-a_{5} a_{7}$, o ponto singular $Q$ é de tipo $A_{1}$.

b) Se $a_{2}=-a_{5} a_{7}$, temos que:

i) Se $a_{2} \neq 0$, para valores genéricos, $Q$ é ponto singular de tipo $A_{2}$.

ii) $S e a_{2}=0$ e $a_{5} \neq 0$ ou $a_{7} \neq 0, Q$ pode ser de tipo $A_{2}, A_{3}, A_{4}$ ou $A_{5}$.

iii) Se $a_{2}=a_{5}=a_{7}=0$, a singularidade é não isolada de tipo $A_{\infty}$.

Demonstração. Seja $F$ a homogenização de $f$ na nova variável $x_{3}, \log \sigma F$ é dada por:

$$
F=a_{2} x_{2} x_{3}^{2}+x_{3}\left(a_{3} x_{0}^{2}+a_{4} x_{0} x_{1}+a_{5} x_{0} x_{2}+a_{6} x_{1}^{2}+a_{7} x_{1} x_{2}+a_{8} x_{2}^{2}\right)+x_{0}^{3}+
$$
$x_{1}^{3}-x_{0} x_{1} x_{2}$.

É fácil verificar que se $a_{8} \neq 0, f$ é regular no infinito e se $a_{8}=0$, $Q=(0: 0: 1: 0)$ é o único ponto singular de $f$ no infinito. Então seja $a_{8}=0$ e $x_{2}=1$, temos que:

$$
F=a_{2} x_{3}^{2}+x_{3}\left(a_{3} x_{0}^{2}+a_{4} x_{0} x_{1}+a_{5} x_{0}+a_{6} x_{1}^{2}+a_{7} x_{1}\right)+x_{0}^{3}+x_{1}^{3}-x_{0} x_{1} .
$$

Se a Hessiana tem posto máximo, isto é, $a_{2} \neq-a_{5} a_{7}, Q=(0: 0: 1: 0)$ é uma singularidade isolada de tipo $A_{1}$. Os próximos itens são todos obtidos por mudança linear de coordenadas. Seja $a_{2}=-a_{5} a_{7}$, temos que: se $a_{2} \neq 0 \Rightarrow a_{5} a_{7} \neq 0$, a singularidade é de tipo $A_{2}$ para valores genéricos. Agora para provar o item b)(ii) vamos supor que $a_{7} \neq 0$, o outro caso segue de maneira análoga. Neste caso temos que se: $a_{3} \neq-a_{7}$, a singularidade é de tipo $A_{2}$, se $a_{3}=-a_{7}$ e $a_{4} \neq 0$ a singularidade é de tipo $A_{3}$, se $a_{4}=0$ e $a_{6} \neq 0$ a singularidade é de tipo $A_{4}$ e se $a_{6}=0$ a singularidade é de tipo $A_{5}$. Para finalizar se $a_{2}=a_{5}=a_{7}=0$, a singularidade de $f$ é não isolada e é de tipo $A_{\infty}$. 
Neste caso temos a seguinte tabela com relação a classificação de $F$ :

\begin{tabular}{|l|c|c|c|c|}
\hline$a_{8} \neq 0$ & \multicolumn{5}{|c|}{$a_{8}=0$} \\
\hline & $a_{2} \neq-a_{5} a_{7}$ & \multicolumn{4}{|c|}{$a_{2}=-a_{5} a_{7}$} \\
\hline & & $a_{2} \neq 0$ & $a_{2}=0$ e $a_{5} \neq 0$ ou $a_{7} \neq 0$ & $\begin{array}{l}a_{2}= \\
a_{7}=0\end{array} a_{5}=$ \\
\hline$A_{0}$ & $A_{1}$ & $A_{2}$ & $A_{2}, A_{3}, A_{4}$ ou $A_{5}$ & $A_{\infty}$ \\
\hline
\end{tabular}

Tabela 4.1: $f_{3}$ nodal

\subsection{2 $f_{3}$ cuspidal}

Lema 4.12. Por uma mudança de coordenadas lineares podemos supor que $f_{3}=x_{2} x_{1}^{2}-x_{0}^{3}$. Com uma translação podemos considerar $f$ equivalente a $f=a_{1} x_{1}+a_{2} x_{2}+a_{3} x_{0}^{2}+a_{4} x_{0} x_{1}+a_{5} x_{0} x_{2}+a_{6} x_{1}^{2}+a_{7} x_{1} x_{2}+a_{8} x_{2}^{2}+x_{2} x_{1}^{2}-x_{0}^{3}$ Temos as seguintes situações:

a) $S e a_{8} \neq 0$, então $f$ é regular no infinito.

b) Se $a_{8}=0,(0: 0: 1: 0)$ é o único ponto singular no infinito e além disso:

i) $a_{5} \neq 0$, a singularidade é de tipo $A_{1}$.

ii) $a_{5}=0, a_{2} \neq 0$ ou $a_{7} \neq 0$, a singularidade é de tipo $A_{2}$.

iii) $a_{2}=a_{7}=a_{5}=0$ e $a_{1} \neq 0$, a singularidade é de tipo $E_{6}$.

iv) $a_{1}=a_{2}=a_{5}=a_{7}=0$ e $a_{3} \neq 0$, a singularidade é de tipo $J_{1, \infty}$.

v) $a_{1}=a_{2}=a_{3}=a_{5}=a_{7}=0$ e $a_{4} \neq 0$, a singularidade é de tipo $J_{2, \infty}$.

Obs.: Se $a_{1}=a_{2}=a_{3}=a_{4}=a_{5}=a_{7}=0, f$ não é uma singularidadessimples.

Demonstração. Sendo $f_{3}$ cuspidal, podemos considerar $F=a_{1} x_{1} x_{3}^{2}+a_{2} x_{2} x_{3}^{2}+x_{3}\left(a_{3} x_{0}^{2}+a_{4} x_{0} x_{1}+a_{5} x_{0} x_{2}+a_{6} x_{1}^{2}+a_{7} x_{1} x_{2}+a_{8} x_{2}^{2}\right)+$ 
$x_{2} x_{1}^{2}-x_{0}^{3}$.

É fácil verificar que se $a_{8} \neq 0, f$ é regular no infinito e se $a_{8}=0, Q=$ $(0: 0: 1: 0)$ é o único ponto singular no infinito. Então seja $a_{8}=0$ e $x_{2}=1$, temos que

$F=a_{1} x_{1} x_{3}^{2}+a_{2} x_{3}^{2}+x_{3}\left(a_{3} x_{0}^{2}+a_{4} x_{0} x_{1}+a_{5} x_{0}+a_{6} x_{1}^{2}+a_{7} x_{1}\right)+x_{1}^{2}-x_{0}^{3}$.

Primeiramente suponha que a Hessiana da parte de grau 2 tem posto máximo, isto é, se $a_{5} \neq 0$ temos uma singularidade isolada de tipo $A_{1}$. Seja $a_{5}=0$ e $a_{2} \neq 0$ ou $a_{7} \neq 0$, a singularidade é de tipo $A_{2}$. Se $a_{2}=a_{5}=a_{7}=0 \mathrm{e}$ $a_{1} \neq 0$, após completar quadrados com relação aos termos $x_{1}^{2}$ e $x_{1} x_{3}^{2}$ temos uma singularidade isolada de tipo $E_{6}$. Se $a_{1}=a_{2}=a_{5}=a_{7}=0$ e $a_{3} \neq 0$, a singularidade não isolada de $F$ é de tipo $J_{1, \infty}$. Se $a_{1}=a_{2}=a_{3}=a_{5}=a_{7}=0$ e $a_{4} \neq 0$, a singularidade não isolada de $F$ é de tipo $J_{2, \infty}$. Se $a_{1}=a_{2}=a_{3}=$ $a_{4}=a_{5}=a_{7}=0, f$ não é uma singularidade simples.

Neste caso temos a seguinte tabela com relação a classificação de $F$ :

\begin{tabular}{|c|c|c|c|c|c|}
\hline$a_{8} \neq 0$ & \multicolumn{5}{|c|}{$a_{8}=0$} \\
\hline & $a_{5} \neq 0$ & & & $=0$ & \\
\hline & & $\begin{array}{l}a_{2} \neq 0 \text { ou } \\
a_{7} \neq 0\end{array}$ & $\begin{array}{l}a_{2}=a_{7}=0 \mathrm{e} \\
a_{1} \neq 0\end{array}$ & $\begin{array}{l}a_{1}=a_{2}= \\
a_{7}=00 \\
a_{3} \neq 0\end{array}$ & $\begin{array}{l}a_{1}=a_{2}= \\
a_{3}=a_{7}=0 \mathrm{e} \\
a_{4} \neq 0\end{array}$ \\
\hline$A_{0}$ & $A_{1}$ & $A_{2}$ & $E_{6}$ & $J_{1, \infty}$ & $J_{2, \infty}$ \\
\hline
\end{tabular}

Tabela 4.2: $f_{3}$ cuspidal

\subsection{3 $f_{3}$ cônica mais corda}


Lema 4.13. Por uma mudança de coordenadas lineares podemos supor que $f_{3}=x_{0}^{3}+x_{0} x_{1} x_{2}$. Por uma translação $f$ é equivalente a $f=a_{1} x_{1}+a_{2} x_{2}+a_{3} x_{0}^{2}+a_{4} x_{0} x_{1}+a_{5} x_{0} x_{2}+a_{6} x_{1}^{2}+a_{7} x_{1} x_{2}+a_{8} x_{2}^{2}+x_{0}^{3}+x_{0} x_{1} x_{2}$ Então:

a) Se $a_{6} a_{8} \neq 0$, então $f$ é regular no infinito.

b) Se $a_{6} a_{8}=0$, temos que se $a_{8}=0$, o ponto singular é $Q=(0: 0: 1: 0)$. Se $a_{6}=0$, o ponto singular é $R=(0: 1: 0: 0)$. Estes são os únicos pontos singulares no infinito e além disso:

i) $a_{2} \neq-a_{5} a_{7}$, a singularidade é de tipo $A_{1}$.

ii) Se $a_{2}=-a_{5} a_{7}$ e $a_{2} \neq 0$, para valores genéricos, a singularidade é de tipo $A_{2}$.

iii) $a_{2}=0$ e $a_{5} \neq 0$ ou $a_{7} \neq 0$ temos uma singularidade de tipo $A_{2}, A_{3}$ ou $A_{4}$.

iv) $a_{2}=a_{5}=a_{7}=0$ e $a_{1} \neq 0$, a singularidade é de tipo $A_{5}$.

v) $a_{1}=a_{2}=a_{5}=a_{7}=0$, a singularidade é não isolada e é de tipo $A_{\infty}$.

Demonstração. Por uma mudança de coordenadas podemos supor que $f_{3}=$ $x_{0}^{3}+x_{0} x_{1} x_{2}$, assim

$f=a_{0} x_{0}+a_{1} x_{1}+a_{2} x_{2}+a_{3} x_{0}^{2}+a_{4} x_{0} x_{1}+a_{5} x_{0} x_{2}+a_{6} x_{1}^{2}+a_{7} x_{1} x_{2}+a_{8} x_{2}^{2}+$ $x_{0}^{3}+x_{0} x_{1} x_{2}$.

Por uma mudança de coordenadas linear eliminamos o termo $a_{0} x_{0}$ e obtemos

$f=a_{1} x_{1}+a_{2} x_{2}+a_{3} x_{0}^{2}+a_{4} x_{0} x_{1}+a_{5} x_{0} x_{2}+a_{6} x_{1}^{2}+a_{7} x_{1} x_{2}+a_{8} x_{2}^{2}+x_{0}^{3}+x_{0} x_{1} x_{2}$.

Portanto $F$ é dada por 
$F=a_{1} x_{1} x_{3}^{2}+a_{2} x_{2} x_{3}^{2}+x_{3}\left(a_{3} x_{0}^{2}+a_{4} x_{0} x_{1}+a_{5} x_{0} x_{2}+a_{6} x_{1}^{2}+a_{7} x_{1} x_{2}+a_{8} x_{2}^{2}\right)+$ $x_{0}^{3}+x_{0} x_{1} x_{2}$.

É fácil verificar que se $a_{6} a_{8} \neq 0, f$ é regular no infinito. Se $a_{8}=0$, $Q=(0: 0: 1: 0)$ é um ponto singular e se $a_{6}=0, R=(0: 1: 0: 0)$ é ponto singular no infinito. Vamos analisar o que acontece com relação ao ponto $Q=(0: 0: 1: 0)$, o comportamento é análogo para o ponto $R=(0: 1: 0: 0)$. Seja $a_{8}=0$ e $x_{2}=1$, então

$F=a_{1} x_{1} x_{3}^{2}+a_{2} x_{3}^{2}+x_{3}\left(a_{3} x_{0}^{2}+a_{4} x_{0} x_{1}+a_{5} x_{0}+a_{6} x_{1}^{2}+a_{7} x_{1}\right)+x_{0}^{3}+x_{0} x_{1}$.

Se $a_{2} \neq-a_{5} a_{7}$ temos que a singularidade é Morse, ou seja temos uma singularidade isolada de tipo $A_{1}$. Agora vamos considerar o caso em que $a_{2}=-a_{5} a_{7}$. Se $a_{2} \neq 0$ o que implica que $a_{5} a_{7} \neq 0$, fazendo uma mudança de coordenadas temos que a singularidade é de tipo $A_{2}$ para valores genéricos. Agora vamos considerar o caso em que $a_{2}=0$ e $a_{5} \neq 0$ ou $a_{7} \neq 0$. Vamos analisar quando $a_{7} \neq 0$, o outro caso é análogo. Se $a_{3} \neq a_{7}$ a singularidade é de tipo $A_{2}$. Se $a_{3}=a_{7}$ e $a_{1} \neq a_{4} a_{7}$ a singularidade é de tipo $A_{3}$ e se $a_{1}=-a_{4} a_{7}$ e $a_{6} \neq 0$ temos que a singularidade é de tipo $A_{4}$. Para finalizar vamos considerar o caso em que $a_{5}=a_{7}=0$. Se $a_{1} \neq 0$ a singularidade é de tipo $A_{5}$ e se $a_{1} \neq 0$ temos uma singularidade não isolada de tipo $A_{\infty}$.

Quando $f_{3}$ é a cônica mais corda, podemos resumir os resultados da classificação em $Q=(0: 0: 1: 0)$ na seguinte tabela.

Nos Lemas 5.2.4, 5.2.5 e 5.2.6 seguintes consideramos respectivamente as formas normais do triângulo, cônica mais tangente e três retas concorrentes. As demonstrações são feitas analogamente aos casos anteriores, por isso 


\begin{tabular}{|l|c|l|l|l|l|}
\hline$a_{8} \neq 0$ & \multicolumn{5}{|c|}{$a_{8}=0$} \\
\hline & $a_{2} \neq-a_{5} a_{7}$ & \multicolumn{4}{|c|}{$a_{2}=-a_{5} a_{7}$} \\
\hline & & $a_{2} \neq 0$ & $\begin{array}{l}a_{2}=0 \text { e } a_{5} \neq 0 \\
\text { ou } a_{7} \neq 0\end{array}$ & $\begin{array}{l}a_{2}=a_{5}= \\
a_{7}=0 \\
a_{1} \neq 0\end{array}$ & $\begin{array}{l}a_{1}=a_{2}= \\
a_{5}=a_{7}=0\end{array}$ \\
\hline$A_{0}$ & $A_{1}$ & $A_{2}$ & $A_{2}, A_{3}$ ou $A_{4}$ & $A_{5}$ & $A_{\infty}$ \\
\hline
\end{tabular}

Tabela 4.3: $f_{3}$ cônica mais corda

colocamos apenas as tabelas.

\subsection{4 $f_{3}$ triângulo}

Lema 4.14. Por uma mudança de coordenadas lineares podemos supor que $f_{3}=x_{0} x_{1} x_{2}$. Então $f$ é da forma:

$f=a_{0} x_{0}+a_{1} x_{1}+a_{2} x_{2}+a_{3} x_{0}^{2}+a_{4} x_{0} x_{1}+a_{5} x_{0} x_{2}+a_{6} x_{1}^{2}+a_{7} x_{1} x_{2}+a_{8} x_{2}^{2}+x_{0} x_{1} x_{2}$

As seguintes situações se verificam:

a) $S e a_{3} a_{6} a_{8} \neq 0, f$ é regular no infinito.

b) Seja $a_{3} a_{6} a_{8}=0$. Se $a_{8}=0, Q=(0: 0: 1: 0)$ é ponto singular no infinito. Se $a_{6}=0$, temos o ponto singular $R=(0: 1: 0: 0)$ e se $a_{3}=0$,o ponto $S=(1: 0: 0: 0)$ é um ponto singular no infinito. Estes são os únicos pontos singulares no infinito e além disso:

i) $a_{2} \neq-a_{5} a_{7}$, a singularidade é de tipo $A_{1}$.

ii) $a_{2}=a_{5} a_{7}$, com $a_{2} \neq 0$, para valores genéricos, a singularidade é de tipo $A_{2}$.

iii) $a_{2}=0$ e $a_{5} \neq 0$ ou $a_{7} \neq 0$, a singularidade pode ser de tipo $A_{2}, A_{3}$ ou $A_{4}$.

iv) $a_{0}=a_{1}=a_{2}=a_{5}=a_{7}=0$, a singularidade é não isolada e é de tipo $A_{\infty}$. 
Quando $f_{3}$ é o triângulo, podemos resumir os resultados da classificação em $Q=(0: 0: 1: 0)$ com a seguinte tabela.

\begin{tabular}{|l|l|l|l|l|}
\hline$a_{8} \neq 0$ & \multicolumn{4}{|c|}{$a_{8}=0$} \\
\hline & $a_{2} \neq-a_{5} a_{7}$ & \multicolumn{3}{|c|}{$a_{2}=-a_{5} a_{7}$} \\
\hline & & $a_{2} \neq 0$ & $a_{2}=0$ e $a_{5} \neq 0$ ou $a_{7} \neq 0$ & $\begin{array}{l}a_{1}=a_{2}=a_{5}= \\
a_{7}=0\end{array}$ \\
\hline$A_{0}$ & $A_{1}$ & $A_{2}$ & $A_{2}, A_{3}$ ou $A_{4}$ & $A_{\infty}$ \\
\hline
\end{tabular}

Tabela 4.4: $f_{3}$ triângulo

\subsection{5 $f_{3}$ cônica mais tangente}

Lema 4.15. Por uma mudança de coordenadas lineares podemos supor que $f_{3}=x_{2} x_{1}^{2}+x_{0}^{2} x_{1}$. Assim $f=a_{0} x_{0}+a_{1} x_{1}+a_{2} x_{2}+a_{3} x_{0}^{2}+a_{4} x_{0} x_{1}+a_{5} x_{0} x_{2}+a_{6} x_{1}^{2}+a_{7} x_{1} x_{2}+a_{8} x_{2}^{2}+$ $x_{2} x_{1}^{2}+x_{0}^{2} x_{1}$

Temos as seguintes situações:

a) $S e a_{8} \neq 0$, então $f$ é regular no infinito.

b) Se $a_{8}=0,(0: 0: 1: 0)$ é o único ponto singular no infinito e além disso:

i) $a_{5} \neq 0$, a singularidade é de tipo $A_{1}$.

ii) $a_{5}=0$ e $a_{2} \neq \frac{a_{7}^{2}}{4}$, a singularidade é de tipo $A_{2}$.

iii) $a_{5}=0, a_{2}=\frac{a_{7}^{2}}{4}$ e a cúbica em $x_{1}$ e $x_{3}$ é não degenerada a singularidade é de tipo $D_{4}$.

iv) $a_{5}=0, a_{2}=\frac{a_{7}^{2}}{4}$ e a cúbica em $x_{1}$ e $x_{3}$ é degenerada a singularidade é de tipo $D_{5}$.

v) $a_{0}=a_{1}=a_{2}=a_{5}=a_{7}=0$ temos o seguinte: se $a_{3} \neq 0$, a singularidade 
é de tipo $J_{2, \infty}$ e se $a_{3}=0$ e $a_{4} \neq 0$ a singularidade é de tipo $T_{\infty, 4,2}$.

Obtemos as seguintes tabelas quando $f_{3}$ é uma cônica mais tangente.

\begin{tabular}{|l|l|l|l|l|}
\hline$a_{8} \neq 0$ & \multicolumn{5}{|c|}{$a_{8}=0$} \\
\hline & $a_{5} \neq 0$ & \multicolumn{3}{|c|}{$a_{5}=0$} \\
\hline & & $a_{2} \neq \frac{a_{7}^{2}}{4}$ & $a_{2}=\frac{a_{7}^{2}}{4}$ e $\delta\left(f_{3}\left(x_{1}, x_{3}\right)\right) \neq 0$ & $\begin{array}{l}a_{2}=a_{7}^{2} \\
\delta\left(f_{3}\left(x_{1}, x_{3}\right)\right)^{\frac{1}{4}}=0\end{array}$ \\
\hline$A_{0}$ & $A_{1}$ & $A_{2}$ & $D_{4}$ & $D_{5}$ \\
\hline
\end{tabular}

Tabela 4.5: $f_{3}$ cônica mais tangente

\begin{tabular}{|c|l|}
\hline \multicolumn{2}{|c|}{$a_{8}=0$} \\
\hline \multicolumn{2}{|c|}{$a_{2}=\frac{a_{7}^{2}}{4}$} \\
\hline$a_{0}=a_{1}=a_{2}=a_{5}=a_{7}=0$ e $a_{3} \neq 0$ & $\begin{array}{l}a_{0}=a_{1}=a_{2}=a_{3}=a_{5}=0= \\
a_{7}=0 \text { e } a_{3} \neq 0\end{array}$ \\
\hline$J_{2, \infty}$ & $T_{\infty, 4,2}$ \\
\hline
\end{tabular}

Tabela 4.6: Continuação $f_{3}$ cônica mais tangente

\subsection{6 $f_{3}$ três retas concorrentes}

Lema 4.16. Por uma mudança de coordenadas podemos supor que $f_{3}=$ $x_{0}^{3}+x_{1}^{3}$. Por uma translação, temos que $f$ é equivalente a $f=a_{2} x_{2}+a_{3} x_{0}^{2}+a_{4} x_{0} x_{1}+a_{5} x_{0} x_{2}+a_{6} x_{1}^{2}+a_{7} x_{1} x_{2}+a_{8} x_{2}^{2}+x_{0}^{3}+x_{1}^{3}$. Considere $\delta$ o discriminante de $a_{3} x_{0}^{2}+a_{4} x_{0} x_{1}+a_{6} x_{1}^{2}$.

Temos as seguintes condições:

a) Se $a_{8} \neq 0$, então $f$ é regular no infinito.

b) Se $a_{8}=0,(0: 0: 1: 0)$ é o único ponto singular no infinito e além disso 
temos:

i) $a_{5} \neq 0$ ou $a_{7} \neq 0$, a singularidade é de tipo $A_{2}$.

ii) $a_{2} \neq 0$ e $a_{5}=a_{7}=0$, a singularidade é de tipo $D_{4}$.

iii) $a_{2}=a_{5}=a_{7}=0$ e o discriminante $(\delta) \neq 0$ a singularidade é não isolada de tipo $T_{\infty, 3,3}$.

Obs.: Se $a_{2}=a_{5}=a_{7}=0$ e o discriminante $(\delta)=0, f$ não é uma singularidade simples.

Obtemos a seguinte tabela com relação a classificação de $F$, quando $f_{3}$ são três retas concorrentes.

\begin{tabular}{|l|l|l|l|}
\hline$a_{8} \neq 0$ & \multicolumn{3}{|c|}{$a_{8}=0$} \\
\hline & $a_{5} \neq 0$ ou $a_{7} \neq 0$ & $a_{5}=a_{7}=0$ e $a_{2} \neq 0$ & $a_{2}=a_{5}=a_{7}=0, \delta \neq 0$ \\
\hline$A_{0}$ & $A_{2}$ & $D_{4}$ & $T_{\infty, 3,3}$ \\
\hline
\end{tabular}

Tabela 4.7: $f_{3}$ três retas concorrentes

\subsection{7 $f_{3}$ duas retas, uma com multiplicidade 2}

Por uma mudança de coordenadas podemos supor que $f_{3}=x_{0} x_{1}^{2}$, assim

$f=a_{0} x_{0}+a_{1} x_{1}+a_{2} x_{2}+a_{3} x_{0}^{2}+a_{4} x_{0} x_{1}+a_{5} x_{0} x_{2}+a_{6} x_{1}^{2}+a_{7} x_{1} x_{2}+a_{8} x_{2}^{2}+x_{0} x_{1}^{2}$.

Temos que $\sum_{f}^{\infty}=\mathbb{P}^{1}=\left\{\left(x_{0}: 0: x_{2}: 0\right),\left(x_{1}, x_{2}\right) \in \mathbb{P}^{1}(\mathbb{C})\right\}$

Como queremos estudar as singularidades no infinito, vamos fazer $\sum_{f}^{\infty} \cap\left\{f_{2}=\right.$ $0\}$. Fazendo $x_{1}=0$ em $f_{2}$ obtemos $f_{2}=a_{3} x_{0}^{2}+a_{5} x_{0} x_{2}+a_{8} x_{2}^{2}$.

Assim $F$ é dada por 


$$
\begin{aligned}
F & =a_{0} x_{0} x_{3}^{2}+a_{1} x_{1} x_{3}^{2}+a_{2} x_{2} x_{3}^{2}+x_{3}\left(a_{3} x_{0}^{2}+a_{4} x_{0} x_{1}+a_{5} x_{0} x_{2}+a_{6} x_{1}^{2}+a_{7} x_{1} x_{2}+\right. \\
\left.a_{8} x_{2}^{2}\right) & +x_{0} x_{1}^{2} .
\end{aligned}
$$

Vamos estudar as singularidades isoladas, para isto, dividiremos em dois casos:

$1^{\circ}$ caso: $f_{2}$ é não degenerada.

Sem perda de generalidade podemos supor que $a_{3}=0$ e $a_{5} \neq 0$. Assim se $x_{2}=0$, obtemos o ponto singular no infinito $Q=(1: 0: 0: 0)$, ou $x_{0}=\frac{-a_{8}}{a_{5}} x_{2}$, em que o ponto singular no infinito $R=\left(\frac{-a_{8}}{a_{5}}: 0: 1: 0\right)$. Primeiramente vamos analisar o que acontece no ponto $Q$. Seja

$$
F=a_{0} x_{3}^{2}+a_{1} x_{1} x_{3}^{2}+a_{2} x_{2} x_{3}^{2}+a_{4} x_{1} x_{3}+a_{5} x_{2} x_{3}+a_{6} x_{1}^{2} x_{3}+a_{7} x_{1} x_{2} x_{3}+a_{8} x_{2}^{2} x_{3}+x_{1}^{2} .
$$

Com uma mudança linear de coordenadas a singularidade no infinito é de tipo $A_{1}$. Agora vamos analisar o que acontece no ponto singular $R=\left(\frac{-a_{8}}{a_{5}}\right.$ : $0: 1: 0)$.

$F=a_{0} x_{0} x_{3}^{2}+a_{1} x_{1} x_{3}^{2}+a_{2} x_{3}^{2}+a_{4} x_{0} x_{1} x_{3}+a_{5} x_{0} x_{3}+a_{6} x_{1}^{2} x_{3}+a_{7} x_{1} x_{3}+a_{8} x_{3}+x_{0} x_{1}^{2}$

A Hessiana de $F=\left(x_{0}: x_{1}: x_{3}\right)$ no ponto $\left(\frac{-a_{8}}{a_{5}}: 0: 0\right)$ é $2 a_{5} a_{8}$. Como $a_{5} \neq 0$, se $a_{8} \neq 0, R$ é uma singularidade de tipo $A_{1}$. E se $a_{8}=0, R$ é uma singularidade de tipo $A_{k}$, com $2 \leq k \leq 5$.

$2^{\circ}$ caso: $f_{2}$ é degenerada.

Neste caso podemos supor que $a_{5}=0$ e que $a_{8} \neq 0$, pois se $a_{8}=0$, as singularidades são não isoladas. Neste caso existe um único ponto singular duplo que é $Q=(1: 0: 0: 0)$, assim 


$$
F=a_{0} x_{3}^{2}+a_{1} x_{1} x_{3}^{2}+a_{2} x_{2} x_{3}^{2}+a_{4} x_{1} x_{3}+a_{6} x_{1}^{2} x_{3}+a_{7} x_{1} x_{2} x_{3}+a_{8} x_{2}^{2} x_{3}+x_{1}^{2} .
$$

Neste caso se $a_{0} \neq \frac{a_{4}^{2}}{4}, Q$ é uma singularidade de tipo $A_{k}$ com $2 \leq k \leq 5$. Se $a_{0}=\frac{a_{4}^{2}}{4}, a_{8} \neq 0, a_{4} \neq 0$ e $a_{1} \neq \frac{a_{4} a_{6}}{2}, Q$ é uma singularidade de tipo $D_{4}$. Se $a_{8}=0$, é uma singularidade não isolada e não vamos entrar em detalhes neste caso.

\subsection{8 $f_{3}$ uma reta de multiplicidade 3}

Por uma mudança de coordenadas podemos supor que $f_{3}=x_{1}^{3}$, assim

$$
f=a_{0} x_{0}+a_{1} x_{1}+a_{2} x_{2}+a_{3} x_{0}^{2}+a_{4} x_{0} x_{1}+a_{5} x_{0} x_{2}+a_{6} x_{1}^{2}+a_{7} x_{1} x_{2}+a_{8} x_{2}^{2}+x_{1}^{3} .
$$

Por uma translação,

$$
f=a_{0} x_{0}+a_{2} x_{2}+a_{3} x_{0}^{2}+a_{4} x_{0} x_{1}+a_{5} x_{0} x_{2}+a_{6} x_{1}^{2}+a_{7} x_{1} x_{2}+a_{8} x_{2}^{2}+x_{1}^{3} .
$$

Temos que $\sum_{f}^{\infty}=\mathbb{P}^{1}=\left\{\left(x_{0}: 0: x_{2}: 0\right)\right\}$.

Como queremos estudar as singularidades no infinito, vamos fazer $\sum_{f}^{\infty} \cap\left\{f_{2}=\right.$ $0\}$. Fazendo $x_{1}=0$ em $f_{2}$ obtemos $f_{2}=a_{3} x_{0}^{2}+a_{5} x_{0} x_{2}+a_{8} x_{2}^{2}$.

Assim, $F$ é dada por

$$
F=a_{0} x_{0} x_{3}^{2}+a_{2} x_{2} x_{3}^{2}+x_{3}\left(a_{3} x_{0}^{2}+a_{4} x_{0} x_{1}+a_{5} x_{0} x_{2}+a_{6} x_{1}^{2}+a_{7} x_{1} x_{2}+a_{8} x_{2}^{2}\right)+x_{1}^{3} .
$$

Para estudar as singularidades isoladas, dividiremos novamente em dois casos:

$1^{\circ}$ caso: $f_{2}$ é não degenerada.

Sem perda de generalidade podemos supor que $a_{3}=0$ e $a_{5} \neq 0$. Assim se 
$x_{2}=0$, em que o ponto singular no infinito $Q=(1: 0: 0: 0)$, ou $x_{0}=\frac{-a_{8}}{a_{5}} x_{2}$, em que o ponto singular no infinito é $R=\left(\frac{-a_{8}}{a_{5}}: 0: 1: 0\right)$. Primeiramente analisaremos o que acontece no ponto $Q$. Neste caso

$$
F=a_{0} x_{3}^{2}+a_{2} x_{2} x_{3}^{2}+a_{4} x_{1} x_{3}+a_{5} x_{2} x_{3}+a_{6} x_{1}^{2} x_{3}+a_{7} x_{1} x_{2} x_{3}+a_{8} x_{2}^{2} x_{3}+x_{1}^{3}
$$

Com uma mudança linear de coordenadas a singularidade no infinito é de tipo $A_{2}$. Agora vamos analisar o que acontece no ponto singular $R$ :

$$
F=a_{0} x_{0} x_{3}^{2}+a_{2} x_{3}^{2}+a_{4} x_{0} x_{1} x_{3}+a_{5} x_{0} x_{3}+a_{6} x_{1}^{2} x_{3}+a_{7} x_{1} x_{3}+a_{8} x_{3}+x_{1}^{3}
$$

A Hessiana de $F=\left(x_{0}: x_{1}: x_{3}\right)$ no ponto $\left(\frac{-a_{8}}{a_{5}}: 0: 0\right)$ é sempre zero. Como $a_{5} \neq 0, R$ é uma singularidade de tipo $A_{k} \operatorname{com} 2 \leq k \leq 6$.

$2^{\circ}$ caso: $f_{2}$ é degenerada.

Neste caso podemos supor que $a_{5}=0$ e que $a_{8} \neq 0$, pois se $a_{8}=0$ as singularidades são não isoladas. Neste caso existe um único ponto singular duplo que é $Q=(1: 0: 0: 0)$, assim

$$
F=a_{0} x_{3}^{2}+a_{2} x_{2} x_{3}^{2}+a_{4} x_{1} x_{3}+a_{6} x_{1}^{2} x_{3}+a_{7} x_{1} x_{2} x_{3}+a_{8} x_{2}^{2} x_{3}+x_{1}^{3} .
$$

Se $a_{4} \neq 0, Q$ é uma singularidade de tipo $A_{k}$ com $2 \leq k \leq 6$. Se $a_{4}=0$, $a_{0} \neq 0$ e $a_{8} \neq 0 Q$ é uma singularidade de tipo $D_{4}$. Se $a_{8}=0$, é uma singularidade não isolada e não vamos entrar em detalhes neste caso.

\subsection{Regularidade no infinito}

Podemos usar a classificação da seção anterior para analisar o comportamento da fibra especial no infinito. Ou seja, queremos saber se $F$ é regular, ou se existe mudança no comportamento da fibra na vizinhança de $t=0$. 
Para isto consideramos a família $F\left(x_{0}, x_{1}, x_{2}, x_{3}\right)-t x_{3}^{3}=0$. Se $t \notin \operatorname{Atyp}(f)$, então o número de Milnor da fibra genérica $f\left(x_{0}, x_{1}, x_{2}\right)=t$ está bem definido e será indicado por $\mu_{t}^{\infty}$.

As proposições a seguir descrevem este comportamento topológico no infinito dos casos estudados anteriormente.

Nota 4.17. 1) Para singularidades isoladas, vamos denotar por $\lambda$ a diferença entre os números de Milnor da fibra especial e da fibra genérica.

2) $A$ notação $A_{k} \rightarrow A_{k+1}$ significa que a singularidade no infinito saltou de $A_{k}$ para $A_{k+1}$ para algum valor de bifurcação. Para singularidades não isoladas usaremos $*$.

Definição 4.18. Dizemos que a família $F-t x_{3}^{3}=0$ é equisingular em $Q=(0: 0: 1: 0)$ em $t=0$, se o salto $\lambda$ em $t=0$ é zero.

Proposição 4.19. Se $f_{3}$ é nodal, então a família $F-t x_{3}^{3}=0$ é equisingular em $Q=(0: 0: 1: 0)$, exceto nos seguintes casos:

(1) $a_{8}=a_{2}=0$ e $a_{5} \neq 0$ ou $a_{7} \neq 0$.

(2) $a_{8}=a_{2}=a_{5}=a_{7}=0$.

No primeiro caso, se $t=0, Q=(0: 0: 1: 0)$ pode ser uma singularidade de tipo $A_{3}, A_{4}$ ou $A_{5}$. No segundo caso, $Q$ é uma singularidade não isolada de tipo $A_{\infty}$. Em ambos os casos, quando $t \neq 0$, a singularidade isolada é de tipo $A_{2}$.

Demonstração. Seja $F$ como no Lema 4.11. Se $t=0$, pelo mesmo lema temos que:

(1) $a_{2}=a_{5} a_{7}=0$ e $a_{5} \neq 0$ ou $a_{7} \neq 0$, então:

- $Q$ é de tipo $A_{3}$ se $a_{3}=-a_{7}$ e $a_{4} \neq 0$. 
- $Q$ é de tipo $A_{4}$ se $a_{4}=0$ e $a_{6} \neq 0$.

- $Q$ é de tipo $A_{5}$ se $a_{6}=0$.

(2) Se $a_{8}=a_{2}=a_{5}=a_{7}=0$, a singularidade é não isolada de tipo $A_{\infty}$.

Em ambos os casos, quando $t \neq 0$, segue facilmente que a singularidade é de tipo $A_{2}$. É fácil verificar que nos casos menos degenerados, o salto é zero.

A tabela 4.8 resume os saltos quando $f_{3}$ é nodal.

\begin{tabular}{|c|c|}
\hline$(0: 0: 1: 0)$ & $\lambda$ \\
\hline$A_{0}$ & 0 \\
\hline$A_{1}$ & 0 \\
\hline$A_{2}$ & 0 \\
\hline$A_{2} \rightarrow A_{3}$ & 1 \\
\hline$A_{2} \rightarrow A_{4}$ & 2 \\
\hline$A_{2} \rightarrow A_{5}$ & 3 \\
\hline$A_{2} \rightarrow A_{\infty}$ & $*$ \\
\hline
\end{tabular}

Tabela 4.8: $f_{3}$ nodal

Proposição 4.20. Se $f_{3}$ é cuspidal, então a família $F-t x_{3}^{3}=0$ é equisingular em $Q=(0: 0: 1: 0)$, exceto nos casos:

(1) $a_{8}=a_{2}=a_{5}=a_{7}=0$ e $a_{1} \neq 0$. Temos que se $t=0, Q=(0: 0: 1: 0)$ é uma singularidade isolada de tipo $E_{6}$.

(2) $a_{8}=a_{2}=a_{5}=a_{7}=0$ e $a_{1}=0$ e $a_{3} \neq 0, Q$ é uma singularidade não isolada de tipo $J_{1, \infty}$ em $t=0$.

(3) $a_{8}=a_{2}=a_{5}=a_{7}=a_{1}=a_{3}=0$ e $a_{4} \neq 0, Q$ é uma singularidade não isolada de tipo $J_{2, \infty}$ em $t=0$.

Em todos os casos, se $t \neq 0$ a singularidade é de tipo $D_{4}$.

Demonstração. Seja $F$ como no Lema 4.12, pelo mesmo lema se $t=0$, temos 
que:

- Se $a_{8}=a_{2}=a_{5}=a_{7}=0$ e $a_{1} \neq 0$ a singularidade é isolada e de tipo $E_{6}$.

- Se $a_{8}=a_{2}=a_{5}=a_{7}=a_{1}=0$ e $a_{3} \neq 0$ a singularidade é não isolada é é de tipo $J_{1, \infty}$.

- Se $a_{8}=a_{2}=a_{5}=a_{7}=a_{1}=a_{3}=0$ e $a_{4} \neq 0$ a singularidade é não isolada de tipo $J_{2, \infty}$. Em todos os casos, se $t \neq 0$, verifica facilmente que a singularidade é de tipo $D_{4}$. É fácil verificar que nos casos menos degenerados, o salto é zero.

A tabela 4.9 resume os saltos quando $f_{3}$ é cuspidal.

\begin{tabular}{|c|c|}
\hline$(0: 0: 1: 0)$ & $\lambda$ \\
\hline$A_{0}$ & 0 \\
\hline$A_{1}$ & 0 \\
\hline$A_{2}$ & 0 \\
\hline$D_{4} \rightarrow E_{6}$ & 2 \\
\hline$D_{4} \rightarrow J_{1, \infty}$ & $*$ \\
\hline$D_{4} \rightarrow J_{2, \infty}$ & $*$ \\
\hline
\end{tabular}

Tabela 4.9: $f_{3}$ cuspidal

Proposição 4.21. Se $f_{3}$ é cônica mais corda, então a família $F-t x_{3}^{3}=0$ é equisingular em $Q=(0: 0: 1: 0)$ exceto nos casos:

(1) $a_{8}=a_{2}=a_{3}=0$ e $a_{5} \neq 0$ ou $a_{7} \neq 0$.

(2) $a_{8}=a_{2}=a_{3}=a_{5}=a_{7}=0$.

No primeiro caso, se $t=0, Q$ é uma singularidade de tipo $A_{3}$ ou $A_{4}$. No segundo caso, se $t=0$ e $a_{1} \neq 0, Q$ é de tipo $A_{5}$ e se $a_{1}=0, Q$ é uma singularidade não isolada de tipo $A_{\infty}$. Em ambos os casos, se $t \neq 0$, a singularidade é isolada e de tipo $A_{2}$.

Demonstração. Seja $F$ como no Lema 4.13, pelo mesmo lema temos que se 
$t=0$, então:

- Se $a_{8}=a_{2}=0$ e $a_{5} \neq 0$ ou $a_{7} \neq 0$, por uma mudança de coordenadas lineares adequada se $a_{1} \neq a_{4} a_{7}, Q$ é de tipo $A_{3}$.

- Se $a_{8}=a_{2}=0, a_{1}=a_{4} a_{7}$ e $a_{6} \neq 0, Q$ é de tipo $A_{4}$.

- Se $a_{8}=a_{2}={ }_{6}=0, a_{1}=a_{4} a_{7}$ e $a_{1} \neq 0, Q$ é de tipo $A_{5}$.

- Se $a_{8}=a_{1}=a_{2}=a_{3}=a_{5}=a_{7}=0, Q=(0: 0: 1: 0)$ é uma singularidade não isolada de tipo $A_{\infty}$.

Em todos os casos, se $t \neq 0$, a singularidade é de tipo $A_{2}$. É fácil verificar que nos casos menos degenerados, o salto é zero.

A tabela 4.10 resume os saltos quando $f_{3}$ é cônica mais corda.

\begin{tabular}{|c|c|}
\hline$(0: 0: 1: 0)$ & $\lambda$ \\
\hline$A_{0}$ & 0 \\
\hline$A_{1}$ & 0 \\
\hline$A_{2}$ & 0 \\
\hline$A_{2} \rightarrow A_{3}$ & 1 \\
\hline$A_{2} \rightarrow A_{4}$ & 2 \\
\hline$A_{2} \rightarrow A_{5}$ & 3 \\
\hline$A_{2} \rightarrow A_{\infty}$ & $*$ \\
\hline
\end{tabular}

Tabela 4.10: $f_{3}$ cônica mais corda

Proposição 4.22. Se $f_{3}$ é cônica mais tangente, então a família $F-t x_{3}^{3}=0$ é equisingular em $Q=(0: 0: 1: 0)$ exceto nos seguintes casos:

(1) $a_{5}=0, a_{2}=\frac{a_{7}^{2}}{4}, \delta\left(f_{3}\left(x_{1}, x_{3}\right)\right)=0$.

(2) $a_{0}=a_{1}=a_{2}=a_{5}=a_{7}=0$ e $a_{3} \neq 0$.

(3) $a_{0}=a_{1}=a_{2}=a_{5}=a_{7}=a_{3}=0$ e $a_{4} \neq 0$.

Demonstração. Pelo Lema 4.15 se $t=0$, então: 
- Se $a_{5}=0, a_{2}=\frac{a_{7}^{2}}{4}, \delta\left(f_{3}\left(x_{1}, x_{3}\right)\right)=0, Q$ é de tipo $D_{5}$.

- Se $a_{0}=a_{1}=a_{2}=a_{5}=a_{7}=0$ e $a_{3} \neq 0$, a singularidade é nao isolada e é de tipo $J_{1, \infty}$.

- Se $a_{0}=a_{1}=a_{2}=a_{5}=a_{7}=a_{3}=0$ e $a_{4} \neq 0$, a singularidade é não isolada e é de tipo $T_{\infty, 4,2}$. Em todos os casos, se $t \neq 0$ a singularidade é de tipo $D_{4}$. É fácil verificar que os casos menos degenerados, o salto é zero.

A tabela 4.11 resume os saltos quando $f_{3}$ é cônica mais tangente.

\begin{tabular}{|c|c|}
\hline$(0: 0: 1: 0)$ & $\lambda$ \\
\hline$A_{0}$ & 0 \\
\hline$A_{1}$ & 0 \\
\hline$A_{2}$ & 0 \\
\hline$D_{4}$ & 0 \\
\hline$D_{4} \rightarrow D_{5}$ & 1 \\
\hline$D_{4} \rightarrow J_{1, \infty}$ & $*$ \\
\hline$D_{4} \rightarrow T_{\infty, 4,2}$ & $*$ \\
\hline
\end{tabular}

Tabela 4.11: $f_{3}$ cônica mais tangente

Proposição 4.23. Se $f_{3}$ é triângulo, então a família $F-t x_{3}^{3}=0$ é equisingular em $Q=(0: 0: 1: 0)$ exceto nos casos:

(1) $a_{8}=a_{2}=0, a_{2}=-a_{5} a_{7}, a_{5} \neq 0$ ou $a_{7} \neq 0$.

(2) $a_{0}=a_{1}=a_{2}=a_{5}=a_{7}=a_{8}=0$.

No primeiro caso, se $t=0, Q=(0: 0: 1: 0)$ pode ser uma singularidade isolada de tipo $A_{3}$ ou $A_{4}$. No segundo caso, se $t=0, Q$ é uma singularidade de tipo $A_{\infty}$. Em ambos os casos, se $t \neq 0$, a singularidade é isolada e é de tipo $A_{2}$.

Demonstração. Pelo Lema 4.14, quando $t=0$, então:

- Se $a_{8}=a_{2}=0, a_{2}=-a_{5} a_{7}, a_{5} \neq 0$ ou $a_{7} \neq 0, a_{0}=a_{3} a_{7}$ e $a_{1} \neq 0 Q$ é de 
tipo $A_{3}$.

- Se $a_{8}=a_{2}=a_{1}=0$ e $a_{4} \neq a_{6} a_{7} Q$ é de tipo $A_{4}$.

- Se $a_{0}=a_{1}=a_{2}=a_{5}=a_{7}=a_{8}=0$, a singularidade é não isolada de tipo $A_{\infty}$

Em ambos os casos, se $t \neq 0$, segue direto que a singularidade é de tipo $A_{2}$. É fácil verificar que nos casos menos degenerados, o salto é zero.

A tabela 4.12 resume os saltos quando $f_{3}$ é triângulo.

\begin{tabular}{|c|c|}
\hline$(0: 0: 1: 0)$ & $\lambda$ \\
\hline$A_{0}$ & 0 \\
\hline$A_{1}$ & 0 \\
\hline$A_{2}$ & 0 \\
\hline$A_{2} \rightarrow A_{3}$ & 1 \\
\hline$A_{2} \rightarrow A_{4}$ & 2 \\
\hline$A_{2} \rightarrow A_{\infty}$ & $*$ \\
\hline
\end{tabular}

Tabela 4.12: $f_{3}$ triângulo

Proposição 4.24. Se $f_{3}$ são três retas concorrentes, então a familia $F-$ $t x_{3}^{3}=0$ é equisingular em $Q=(0: 0: 1: 0)$ exceto nos casos:

(1) $a_{8}=a_{5}=a_{7}=0$ e se $a_{2} \neq 0$.

(2) $a_{8}=a_{5}=a_{7}=A_{2}=0$ e $\delta\left(a_{3} x_{0}^{2}+a_{4} x_{0} x_{1}+a_{6} x_{1}^{2}\right) \neq 0$.

No primeiro caso, se $t=0, Q=(0: 0: 1: 0)$ é de tipo $D_{4}$. No segundo caso, se $t=0, Q$ é uma singularidade não isolada de tipo $T_{\infty, 3,3}$. Em ambos os casos, se $t \neq 0$, a singularidade isolada é de tipo $A_{2}$.

Demonstração. Seja $f$ como no Lema 4.16, pelo mesmo lema temos que:

- Se $a_{8}=a_{5}=a_{7}=0$ e $a_{2} \neq 0$, se $t=0, Q=(0: 0: 1: 0)$ é de tipo $D_{4}$.

- Se $a_{8}=a_{5}=a_{7}=a_{2}=0$ e se o discriminate $\delta\left(a_{3} x_{0}^{2}+a_{4} x_{0} x_{1}+a_{6} x_{1}^{2}\right)$ é diferente de zero, $Q$ é uma singularidade não isolada de tipo $T_{\infty, 3,3}$. 
Em ambos os casos, se $t \neq 0$ segue facilmente que a singularidade é de tipo $A_{2}$. É fácil verificar que nos casos menos degenerados, o salto é zero. - A tabela 4.13 resume os saltos quando $f_{3}$ são três retas concorrentes.

\begin{tabular}{|c|c|}
\hline$(0: 0: 1: 0)$ & $\lambda$ \\
\hline$A_{0}$ & 0 \\
\hline$A_{2}$ & 0 \\
\hline$A_{2} \rightarrow D_{4}$ & 2 \\
\hline$A_{2} \rightarrow T_{\infty, 3,3}$ & $*$ \\
\hline
\end{tabular}

Tabela 4.13: $f_{3}$ três retas concorrentes 


\section{Capítulo 5}

\section{Classificação de superfícies cúbicas projetivas}

\subsection{Introdução}

O objetivo desse capítulo é classificar singularidades no infinito de polinômios $f: \mathbb{C}^{3} \rightarrow \mathbb{C}, g r(f)=3$, em que $g r$ indica o grau do polinômio $f$. Vamos refinar a classificação das singularidades no infinito obtida por Bruce e Wall em [11], para obter uma descrição mais detalhada das singularidades no infinito de polinômios de grau 3 da forma $f=f_{2}+f_{3}$, em que $f_{2}$ é a parte homogênea de grau 2 e $f_{3}$ é a parte homogênea de grau 3. Esta classificação será importante no estudo da topologia da fibra, através do invariante $\Delta_{2}(f)$ que será apresentado no próximo capítulo .

\subsection{Cúbicas singulares em $\mathbb{P}^{3}$}

As superfícies cúbicas não singulares em $\mathbb{P}^{3}$ foram classificadas por Segre [37]. Uma classificação das superfícies cúbicas singulares foi obtida por Bruce e Wall em [11]. Nesta seção descrevemos os principais resultados de Bruce e 
Wall em [11] e apresentamos um refinamento da classificação de superfícies cúbicas singulares por eles obtida.

Seja $P$ um ponto singular da superfície cúbica projetiva $V$. Podemos escolher coordenadas homogêneas $\left(x_{0}: x_{1}: x_{2}: x_{3}\right)$ e considerar $P=(0: 0$ : $0: 1)$, logo a equação da superfície toma a seguinte forma

$$
F=x_{3} f_{2}\left(x_{0}, x_{1}, x_{2}\right)+f_{3}\left(x_{0}, x_{1}, x_{2}\right)
$$

em que $f_{i}$ é homogêneo de grau $i$. Então $V=\left\{\left(x_{0}: x_{1}: x_{2}: x_{3}\right) \in\right.$ $\left.\mathbb{P}^{3} \mid F\left(x_{0}, x_{1}, x_{2}, x_{3}\right)=0\right\}$.

Obtemos as coordenadas locais afins em $P$ tomando $x_{3}=1$. O primeiro invariante da classificação é o rank da forma quadrática $f_{2}$. Temos o seguinte [11]:

CASO A: rank 3, P é chamado nó cônico (conic node).

CASO B: rank 2, $P$ é chamado binó (binode).

CASO C: rank 1, $P$ é chamado nó degenerado (unode).

CASO D: rank 0, $P$ é chamado ponto triplo (triple point).

Para cada um dos casos A, B, C e D, vamos apresentar nas próximas subseções a classificação das singularidades da hipersuperfície projetiva $F=$ 0, obtida por Bruce e Wall e o refinamento que obtivemos para o caso correspondente.

\section{$5.3 \quad \mathrm{P}$ é nó cônico}

Primeiro note que $P$ é um nó cônico se $P$ é uma singularidade de tipo $A_{1}$, ou seja uma singularidade de Morse. Logo a Hessiana de $f_{2}$ em $P$ é não singular e portanto podemos fazer uma mudança de coordenadas linear de tal forma 
que $f_{2}=x_{1}^{2}-x_{0} x_{2}$.

Podemos ver que as raízes comuns de $f_{2}=f_{3}=0$ correspondem a retas de $V$ que passam por $P$ e reciprocamente. De fato, se $Q \neq P$ é tal que $f_{2}(Q)=f_{3}(Q)=0$, como $f_{2}$ e $f_{3}$ são homogêneos, temos que $f_{2}(t Q)=f_{3}(t Q)=0, t \neq 0$.

O próximo lema mostra que a classificação da superfície $F=0$ se reduz à classificação do feixe gerado por $f_{2}=0$ e $f_{3}=0$.

Lema 5.1. (Lema 2 parte (a), [11]) Seja $F=x_{3}\left(x_{1}^{2}-x_{0} x_{2}\right)+f_{3}\left(x_{0}, x_{1}, x_{2}\right)$ e $G=x_{3}\left(x_{1}^{2}-x_{0} x_{2}\right)+g_{3}\left(x_{0}, x_{1}, x_{2}\right)$. Então $F=0$ e $G=0$ são superfícies cúbicas projetivamente equivalentes por um isomorfismo que fixa $P$, se e somente se, $f_{3}\left(\theta^{2}, \theta \phi, \phi^{2}\right)$ e $g_{3}\left(\theta^{2}, \theta \phi, \phi^{2}\right)$ são sêxticas binárias equivalentes.

Demonstração. Se $F=0$ e $G=0$ são projetivamente equivalentes, então a mudança de coordenadas leva as 6 retas (contadas com multiplicidade) que passam por $P$, dadas por $f_{3}\left(\theta^{2}, \theta \phi, \phi^{2}\right)=0$ nas 6 retas que também passam por $P$, dadas por $g_{3}\left(\theta^{2}, \theta \phi, \phi^{2}\right)=0$, preservando multiplicidades. Portanto, as sêxticas binárias são equivalentes.

Reciprocamente, se $f_{3}\left(\theta^{2}, \theta \phi, \phi^{2}\right)$ e $g_{3}\left(\theta^{2}, \theta \phi, \phi^{2}\right)$ são sêxticas binárias equivalentes, então a mudança de coordenadas na reta projetiva $(\theta: \phi)$ induz um automorfismo da cônica $x_{1}^{2}-x_{0} x_{2}$, que transforma a cúbica $g_{3}$ numa cúbica $g_{3}^{\prime}$, com a propriedade que as interseções $g_{3}^{\prime}=f_{2}=0$ coincidem com as interseções $f_{3}=f_{2}=0$. Então podemos escrever $f_{3}=\lambda g_{3}^{\prime}+\left(x_{1}^{2}-x_{0} x_{2}\right) l$ para algum $\lambda \neq 0$ e uma forma linear $l$. Uma mudança de coordenada da forma $x_{3}^{\prime}=x_{3}-l$ prova o resultado. 
Seja

$f_{3}\left(x_{0}, x_{1}, x_{2}\right)=a_{0} x_{0}^{3}+a_{1} x_{0}^{2} x_{1}+\left(b_{1} x_{0}^{2} x_{2}+b_{2} x_{0} x_{1}^{2}\right)+\left(b_{3} x_{0} x_{1} x_{2}+b_{4} x_{1}^{3}\right)+\left(b_{5} x_{1}^{2} x_{2}+\right.$ $\left.b_{6} x_{0} x_{2}^{2}\right)+a_{5} x_{1} x_{2}^{2}+a_{6} x_{2}^{3}$.

Assim a equação da sêxtica se reduz a $f_{3}\left(\theta^{2}, \theta \phi, \phi^{2}\right)=\sum_{0}^{6} a_{i} \theta^{6-i} \phi^{i}$.

Por uma mudança de coordenadas podemos supor que o ponto singular $Q \neq P$ é $Q=(0: 0: 1: 0)$.

Assim derivando $f_{3}$ com relação às variáveis $x_{0}, x_{1}$ e $x_{2}$ e colocando a condição que $(0: 0: 1: 0)$ é ponto singular temos que $b_{6}=a_{5}=a_{6}=0$.

Logo temos que

$f_{3}\left(x_{0}, x_{1}, x_{2}\right)=a_{0} x_{0}^{3}+a_{1} x_{0}^{2} x_{1}+\left(b_{1} x_{0}^{2} x_{2}+b_{2} x_{0} x_{1}^{2}\right)+\left(b_{3} x_{0} x_{1} x_{2}+b_{4} x_{1}^{3}\right)+$ $b_{5} x_{1}^{2} x_{2}$. Assim,

$f_{3}\left(\theta^{2}, \theta \phi, \phi^{2}\right)=\theta^{2}\left(a_{0} \theta^{4}+a_{1} \theta^{3} \phi+\left(b_{1}+b_{2}\right) \theta^{2} \phi^{2}+\left(b_{3}+b_{4}\right) \theta \phi^{3}+b_{5} \phi^{4}\right)$.

Temos ainda o seguinte resultado.

Lema 5.2. ( Lema 2 parte (b) e (c), [11])(i) Para cada singularidade $Q \neq P$, a reta $\overline{P Q}$ está em $V$, então temos uma raiz comum de $f_{2}$ e $f_{3}$, isto é, $f_{3}\left(\theta^{2}, \theta \phi, \phi^{2}\right)=0$, cuja multiplicidade é maior ou igual a 2 .

(ii) Cada raiz determina uma reta l passando por $P$ em $V$. Se a raiz tem multiplicidade maior ou igual a 2, existe precisamente um ponto singular em $l$.

(iii) Uma raiz $k$-upla de $f_{3}\left(\theta^{2}, \theta \phi, \phi^{2}\right)=0$ corresponde a uma singularidade $A_{k-1}$. 
Demonstração. i) Já vimos que a reta $\overline{P Q}$ está em $V$. Por uma mudança de coordenadas, podemos supor que o ponto singular seja $Q=(0: 0: 1: 0)$ e portanto em $f_{3}$ os termos $x_{0} x_{2}^{2}, x_{1} x_{2}^{2}$ e $x_{2}^{3}$ são nulos, logo $f_{3}\left(\theta^{2}, \theta \phi, \phi^{2}\right)$ tem $\theta^{2}$ como um fator, isto é, a sêxtica $f_{3}\left(\theta^{2}, \theta \phi, \phi^{2}\right)$ tem uma raiz com multiplicidade maior ou igual a 2 .

ii) Suponha que $(\theta: \phi)=(0: 1)$ é a raiz múltipla de $f_{3}\left(\theta^{2}, \theta \phi, \phi^{2}\right)=0$, então $Q=(0: 0: 1: 0)$ é um ponto singular de $V$. Além disso, não existem outros pontos singulares ao longo da reta $\overline{P Q}$ já que

$$
\frac{\partial F}{\partial x_{0}}=-x_{2} x_{3} \frac{\partial f_{3}}{\partial x_{0}}
$$

se anula nesta reta somente em $P$ e $Q$.

(iii) Suponha que a raiz múltipla da sêxtica é $(0: 1)$. Temos que $(0: 1)$ é raiz de multiplicidade $k$, se e somente se, $a_{6}=\ldots=a_{7-k}=0$ e $a_{6-k} \neq 0$ e a singularidade é em $Q=(0: 0: 1: 0)$. Tomando $x_{2}=1$ temos

$$
x_{3} x_{1}^{2}-x_{3} x_{0}+a_{0} x_{0}^{3}+a_{1} x_{0}^{2} x_{1}+a_{2} x_{0} x_{1}^{2}+a_{3} x_{1}^{3}+a_{4} x_{1}^{2},
$$

fazendo a mudança de coordenadas

$$
x_{3}=x_{3}^{\prime}+a_{0}\left(x_{0}^{2}+x_{0}^{2} x_{1}+x_{1}^{4}\right)+a_{1}\left(x_{0} x_{1}+x_{1}^{3}\right)+a_{2} x_{1}^{2}, x_{0}=x_{0}{ }^{\prime}+x_{1}^{2} \mathrm{em}
$$

obtemos

$$
-x_{3}^{\prime} x_{0}^{\prime}+a_{0} x_{1}^{6}+a_{1} x_{1}^{5}+a_{2} x_{1}^{4}+a_{3} x_{1}^{3}+a_{4} x_{1}^{2}
$$

e segue o resultado.

Das informações acima podemos observar o seguinte resultado:

\section{Nota 5.3.}


(1) $(0: 0: 1: 0)$ é uma singularidade de $F \Leftrightarrow(\theta: \phi)=(0: 1)$ é uma raiz de $f_{3}\left(\theta^{2}, \theta \phi, \phi^{2}\right)=0$ com multiplicidade maior ou igual a 2 .

(2) $(\theta: \phi)=(0: 1)$ é uma raiz com multiplicidade $2 \Leftrightarrow b_{5} \neq 0$.

\subsubsection{Descrevendo a classificação}

Nota 5.4. $a^{b}$ significa $b$ raízes de multiplicidade a.

Como uma consequência dos Lemas 5.1 e 5.2 as singularidades de $F$ são determinadas pelas partições de 6 , dadas pelas soluções com multiplicidade maior ou igual a 2 de $f_{2}=f_{3}=0$.

Como quaisquer três pontos da reta projetiva podem ser levados por uma mudança de coordenadas em $(1: 0: 0: 0),(0: 1: 0: 0)$ e $(0: 0: 1: 0)$, enquanto quatro pontos quaisquer definem um cross-ratio, podemos listar o número de parâmetros de que cada tipo depende.

\begin{tabular}{|c|c|c|c|c|c|c|c|c|c|l|l|}
\hline partição & $1^{6}$ & $21^{4}$ & $31^{3}$ & $2^{2} 1^{2}$ & $41^{2}$ & 321 & $2^{3}$ & 51 & 42 & 33 & 6 \\
\hline singul. & $A_{1}$ & $2 A_{1}$ & $A_{1} A_{2}$ & $3 A_{1}$ & $A_{1} A_{3}$ & $2 A_{1} A_{2}$ & $4 A_{1}$ & $A_{1} A_{4}$ & $2 A_{1} A_{3}$ & $A_{1} 2 A_{2}$ & $A_{1} A_{5}$ \\
\hline $\mathrm{n}^{\circ}$ de Par. & 3 & 2 & 1 & 1 & 0 & 0 & 0 & 0 & 0 & 0 & 0 \\
\hline
\end{tabular}

Tabela 5.1: $f_{2}$ é nó cônico

\section{INTERPRETANDO A TABELA}

$1^{6} \rightarrow$ Seis raízes simples, isto é, $F$ não tem singularidade no infinito, a única singularidade de $F$ é $P$. Como temos seis raízes distintas, o número de parâmetros é 3 .

$21^{4} \rightarrow$ Uma raiz de multiplicidade 2 e quatro raízes simples $\left(b_{5} \neq 0\right)$. Aqui os pontos singulares são $P$ e $Q$, ambos são singularidades $A_{1}$. Como temos 
cinco raízes (sem contar multiplicidade) temos 2 parâmetros.

$31^{3} \rightarrow$ Uma raiz de multiplicidade 3 e três raízes simples $\left(b_{5}=0, b_{3} \neq-b_{4}\right)$. Aqui temos os pontos singulares $P$ e $Q$, em que $P$ é uma singularidade $A_{1}$ e $Q$ uma singularidade $A_{2}$. Como temos quatro raízes (sem contar multiplicidade), há apenas 1 parâmetro.

$2^{2} 1^{2} \rightarrow$ Duas raízes de multiplicidade 2 e duas raízes simples $\left(b_{5} \neq 0\right)$. Aqui os pontos singulares são $P$ e $Q$ e um outro ponto singular, digamos $R$, onde ambos são singularidades $A_{1}$. Como há quatro raízes (sem contar multiplicidade) temos 1 parâmetro.

$41^{2} \rightarrow$ Uma raiz de multiplicidade 4 e duas raízes simples $\left(b_{5}=0, b_{3}=\right.$ $\left.-b_{4}, b_{1} \neq-b_{2}\right)$. Neste caso os pontos singulares são $P$ e $Q$, onde $P$ é uma singularidade $A_{1}$ e $Q$ uma singularidade $A_{3}$. Como temos três raízes (sem contar multiplicidade) não há parâmetros.

$321 \rightarrow$ Uma raiz de multiplicidade 3 , uma raiz de multiplicidade 2 e uma raiz simples. Aqui temos os pontos singulares $P$ e $Q$ e um outro ponto singular, digamos $R$, em que $P$ e $Q$ são singularidades $A_{1}$ e $R$ uma singularidade $A_{2}$. Como são três raízes (sem contar multiplicidade) não há parâmetros.

$2^{3} \rightarrow$ Três raízes de multiplicidade 2 . Aqui temos os pontos singulares $P$ e $Q$ e outros dois pontos singulares, digamos $R$ e $S$, onde ambos são singularidades $A_{1}$. Como temos três raízes (sem contar multiplicidade) não tem parâmetros.

$51 \rightarrow$ Uma raiz de multiplicidade 5 e uma raiz simples $\left(b_{5}=0, b_{3}=-b_{4}, b_{1}=\right.$ $\left.-b_{2}, a_{1} \neq 0\right)$. Neste caso os pontos singulares são $P$ e $Q$, onde $P$ é uma singu- 
laridade $A_{1}$ e $Q$ uma singularidade $A_{4}$. Como temos duas raízes (sem contar multiplicidade) não há parâmetros.

$42 \rightarrow$ Uma raiz de multiplicidade 4 e uma raiz de multiplicidade 2. Aqui temos os pontos singulares $P$ e $Q$ e um outro ponto singular, digamos $R$, onde $P$ e $Q$ são singularidades $A_{1}$ e $R$ singularidade $A_{3}$. Como temos duas raízes (sem contar multiplicidade) não há parâmetros.

$33 \rightarrow$ Duas raízes de multiplicidade 3. Aqui temos os pontos singulares $P$ e $Q$ e um outro ponto singular, digamos $R$, onde $P$ é uma singularidade $A_{1}, Q$ e $R$ singularidades $A_{2}$. Como são duas raízes (sem contar multiplicidade) não tem parâmetros.

$6 \rightarrow$ Uma raiz de multiplicidade $\left.6, b_{5}=0, b_{3}=-b_{4}, b_{1}=-b_{2}, a_{1}=0, a_{0} \neq 0\right)$. Aqui os pontos singulares são $P$ e $Q$, onde $P$ é uma singularidades $A_{1}$ e $Q$ uma singularidade $A_{4}$. Como temos uma única raiz (sem contar multiplicidade) não há parâmetros.

\subsubsection{Refinamento da classificação quando $f_{2}$ é nó cônico}

Nas próximas proposições apresentamos um refinamento da classificação anterior, em termos da classificação das singularidades de $f_{3}$. Esta classificação será útil no estudo da topologia da fibra especial que faremos no capítulo 6 .

Seja

$f_{3}\left(x_{0}, x_{1}, x_{2}\right)=a_{0} x_{0}^{3}+a_{1} x_{0}^{2} x_{1}+\left(b_{1} x_{0}^{2} x_{2}+b_{2} x_{0} x_{1}^{2}\right)+\left(b_{3} x_{0} x_{1} x_{2}+b_{4} x_{1}^{3}\right)+b_{5} x_{1}^{2} x_{2}$.

Fazendo $x_{2}=1$ temos 


$$
f_{3}\left(x_{0}, x_{1}, 1\right)=\overbrace{a_{0} x_{0}^{3}+a_{1} x_{0}^{2} x_{1}+b_{2} x_{0} x_{1}^{2}+b_{4} x_{1}^{3}}^{\widetilde{f}_{3}}+\overbrace{b_{1} x_{0}^{2}+b_{3} x_{0} x_{1}+b_{5} x_{1}^{2}}^{\widetilde{f}_{2}},
$$

em que estamos considerando $\widetilde{f}_{2}$ e $\widetilde{f}_{3}$ as partes de grau 2 e 3 respectivamente de $f_{3}$ nas variáveis $\left(x_{0}: x_{1}: 1: 0\right)$. Representaremos o discriminante de $\widetilde{f}_{2}$ por $\delta\left(\widetilde{f}_{2}\right)=b_{3}^{2}-4 b_{1} b_{5}$ e $\operatorname{Res}(\alpha, \beta)$ denota a resultante dos polinômios $\alpha$ and $\beta$.

Nos casos abaixo, fixamos primeiro o tipo de singularidade de $f_{2}$ em $P$, e chamamos $f_{2}$ com o mesmo nome desta singularidade em $P$. Em todos os casos $F=x_{3} f_{2}+f_{3}$.

\section{Proposição 5.5. Seja}

$f_{3}\left(\theta^{2}, \theta \phi, \phi^{2}\right)=\theta^{2} \overbrace{\left(a_{0} \theta^{4}+a_{1} \theta^{3} \phi+\left(b_{1}+b_{2}\right) \theta^{2} \phi^{2}+\left(b_{3}+b_{4}\right) \theta \phi^{3}+b_{5} \phi^{4}\right)}^{\beta}$. Então, $F$ é de tipo $21^{4} \Leftrightarrow b_{5} \neq 0, \operatorname{Res}\left(\beta, \frac{\partial \beta}{\partial \phi}\right) \neq 0$. Além disso:

(a) $f_{3}$ é nodal $\Leftrightarrow \delta\left(\widetilde{f}_{2}\right) \neq 0$.

(b) $f_{3}$ é cuspidal $\Leftrightarrow \delta\left(\widetilde{f}_{2}\right)=0$ e $f_{3}$ é irredutível.

(c) $f_{3}$ é cônica mais tangente $\Leftrightarrow \delta\left(\widetilde{f}_{2}\right)=0$ e $f_{3}$ é redutível.

Demonstração. A primeira equivalência é imediata, pois a condição $b_{5} \neq 0$ garante que a multiplicidade da raiz $(\theta: \phi)=(0: 1)$ é 2 e a condição $\operatorname{Res}\left(\beta, \frac{\partial \beta}{\partial \phi}\right) \neq 0$ que as demais raízes são simples.

Já que $Q$ é o único ponto singular no infinito, então $f_{3}$ é nodal, cuspidal ou cônica mais tangente.

Note que, apesar de $f_{3}$ ter uma única singularidade não é possível obter três retas concorrentes, pois $b_{5} \neq 0$. 
Proposição 5.6. Seja

$f_{3}\left(\theta^{2}, \theta \phi, \phi^{2}\right)=\theta^{3} \overbrace{\left(a_{0} \theta^{3}+a_{1} \theta^{2} \phi+\left(b_{1}+b_{2}\right) \theta \phi^{2}+\left(b_{3}+b_{4}\right) \phi^{3}\right)}^{\beta_{1}}$. Então, $F$ é de tipo $31^{3} \Leftrightarrow b_{5}=0, b_{3} \neq-b_{4}$ e $\operatorname{Res}\left(\beta_{1}, \frac{\partial \beta_{1}}{\partial \phi}\right) \neq 0$. Além disso:

(a) $f_{3}$ é $\operatorname{nodal} \Leftrightarrow \delta\left(\widetilde{f}_{2}\right) \neq 0$.

(b) $f_{3}$ é cuspidal $\Leftrightarrow \delta\left(\widetilde{f}_{2}\right)=0, \widetilde{f}_{2} \neq 0$ e $f_{3}$ é irredutível.

(c) $f_{3}$ é cônica mais tangente $\Leftrightarrow \delta\left(\widetilde{f}_{2}\right)=0, \widetilde{f}_{2} \neq 0$ e $f_{3}$ é redutível.

(d) $f_{3}$ são três retas concorrentes $\Leftrightarrow \widetilde{f}_{2}=0$.

Demonstração. A primeira equivalência é imediata, pois a condição $b_{5}=$ $0, b_{3} \neq-b_{4}$ garante que a multiplicidade da raiz $(0: 1)$ é 3 e a condição $\operatorname{Res}\left(\beta_{1}, \frac{\partial \beta_{1}}{\partial \phi}\right) \neq 0$ que as demais raízes são simples.

O ponto $Q$ é o único ponto singular no infinito garantindo que $f_{3}$ é nodal, cuspidal, cônica mais tangente ou três retas concorrentes.

As demonstrações das Proposições 5.7, 5.8 e 5.9 são análogas.

Proposição 5.7. Seja $f_{3}\left(\theta^{2}, \theta \phi, \phi^{2}\right)=\theta^{4} \overbrace{\left(a_{0} \theta^{2}+a_{1} \theta \phi+\left(b_{1}+b_{2}\right) \phi^{2}\right)}^{\beta_{2}}$. Então, $F$ é de tipo $41^{2} \Leftrightarrow b_{5}=0, b_{3}=-b_{4} b_{1} \neq-b_{2}$ e Res $\left(\beta_{2}, \frac{\partial \beta_{2}}{\partial \phi}\right) \neq 0$. Além disso:

(a) $f_{3}$ é $\operatorname{nodal} \Leftrightarrow \delta\left(\widetilde{f}_{2}\right) \neq 0$.

(b) $f_{3}$ é cônica mais tangente $\Leftrightarrow \delta\left(\widetilde{f}_{2}\right)=0$ e $\widetilde{f}_{2} \neq 0$.

(c) $f_{3}$ são três retas concorrentes $\Leftrightarrow \widetilde{f}_{2}=0$.

Proposição 5.8. Seja $f_{3}\left(\theta^{2}, \theta \phi, \phi^{2}\right)=\theta^{5} \overbrace{\left(a_{0} \theta+a_{1} \phi\right)}^{\beta_{3}}$. Então, $F$ é de tipo $51 \Leftrightarrow b_{5}=0, b_{3}=-b_{4} b_{1}=-b_{2}$ e $a_{1} \neq 0$. Além disso: 
(a) $f_{3}$ é $\operatorname{nodal} \Leftrightarrow \delta\left(\widetilde{f}_{2}\right) \neq 0$.

(b) $f_{3}$ é cônica mais tangente $\Leftrightarrow \delta\left(\widetilde{f}_{2}\right)=0$ e $\widetilde{f}_{2} \neq 0$.

Proposição 5.9. Seja $f_{3}\left(\theta^{2}, \theta \phi, \phi^{2}\right)=a_{0} \theta^{6}$. Então, $F$ é de tipo $6 \Leftrightarrow b_{5}=$ $0, b_{3}=-b_{4}, b_{1}=-b_{2}, a_{1}=0$ e $a_{0} \neq 0$. Além disso:

(a) $f_{3}$ é nodal $\Leftrightarrow \delta\left(\widetilde{f}_{2}\right) \neq 0$.

(b) $f_{3}$ é cônica mais tangente $\Leftrightarrow \delta\left(\widetilde{f}_{2}\right)=0$ e $\widetilde{f}_{2} \neq 0$.

Segue que $F$ tem dois pontos singulares no infinito, se e somente se, $f_{3}$ é cônica mais corda. Da mesma forma tem três singularidades no infinito, se e somente se, $f_{3}$ é um triângulo como mostram as próximas proposições.

Proposição 5.10. Temos que F tem um dos seguintes tipos: $2^{2} 1^{2}, 321,42$ ou 33, se e somente se, $f_{3}$ é cônica mais corda.

Demonstração. Basta observar que se $F$ é de tipo $2^{2} 1^{2}, 321,42$ ou 33, então temos dois pontos singulares e a única forma cúbica com exatamente dois pontos singulares é a cônica mais corda. Reciprocamente se $f_{3}$ é uma cônica mais uma corda, então temos exatamente dois pontos singulares o que implica que $F$ é do tipo $2^{2} 1^{2}, 321,42$ ou 33 .

Proposição 5.11. Temos que $F$ é de tipo $2^{3} \Leftrightarrow f_{3}$ é um triângulo.

Demonstração. Se $F$ é de tipo $2^{3}$, então temos exatamente 3 pontos singulares o que implica que $f_{3}$ é um triângulo. Reciprocamente se $f_{3}$ é um triângulo, temos 3 pontos singulares o que implica que $F$ é do tipo $2^{3}$.

\section{$5.4 \quad P$ é um binó}

Neste caso $f_{2}=0$ é um par de retas. Por uma mudança de coordenadas podemos supor que $f_{2}=x_{0} x_{1}$. Temos que $P$ é uma singularidade de corank 
1 , isto é, uma singularidade $A_{k}$ para algum $k \geq 2$.

Como $P$ é um binó, fazendo a mudança de coordenadas $x_{3}=X_{3}+x_{0}+$ $x_{1}+x_{2}$, podemos tomar $F$ como

$F=x_{3} x_{0} x_{1}+x_{0}\left(a_{0} x_{0}^{2}+a_{1} x_{0} x_{2}+a_{2} x_{2}^{2}\right)+x_{1}\left(a_{3} x_{1}^{2}+a_{4} x_{1} x_{2}+a_{5} x_{2}^{2}\right)+a_{6} x_{2}^{3}=0$.

Lema 5.12. (Bruce, [11],pp 248) Seja $F=x_{3} x_{0} x_{1}+f_{3}\left(x_{0}, x_{1}, x_{2}\right)$.

(a) Singularidades de $F=0$ diferentes da singularidade $P$, correspondem a uma interseção múltipla de $x_{0} x_{1}=0$ com $f_{3}=0$, fora de $(0: 0: 1) \mathrm{em} \mathbb{P}^{2}$.

(b) Uma interseção k-múltipla fora de (0:0:1) corresponde a uma singularidade $A_{k-1}$.

(c) Se $f_{3}(0: 0: 1) \neq 0, P$ é uma singularidade do tipo $A_{2}$. Se $(0: 0: 1)$ é uma interseção $k_{i}$-múltipla de $x_{i}=0$ com $f_{3}=0, i=0,1$, então $P$ é uma singularidade $A_{k_{0}+k_{1}+1}$ para $\left\{k_{0}, k_{1}\right\}=\{1,1\},\{1,2\},\{1,3\}$.

Demonstração. (a) Considerando $F$ como acima temos que $f_{2}=x_{0} x_{1}=$ $0 \Rightarrow x_{0}=0$ ou $x_{1}=0$. Se $x_{1}=0$ tem uma interseção múltipla com $f_{3}=0$, digamos (1: 0 : 0 ), então $a_{0}=a_{1}=0$ e nós temos uma singularidade em $(1: 0: 0: 0)$. De maneira análoga se $(1: 0: 0: 0)$ é uma singularidade de $F$ temos que $a_{0}=a_{1}=0$ e $x_{1}=0$. Se $x_{0}=0$ o argumento é análogo.

(b) Com o ponto múltiplo como no item (a), seja $x_{0}=1$, então

$$
x_{3} x_{1}+a_{2} x_{2}^{2}+a_{6} x_{2}^{3}+x_{1}\left(a_{3} x_{1}^{2}+a_{4} x_{1} x_{2}+a_{5} x_{2}^{2}\right)
$$

Quando $a_{2} \neq 0, Q=(1: 0: 0: 0)$ é uma singularidade $A_{1}$. Se $a_{2}=0 \mathrm{e}$ $a_{6} \neq 0, Q$ é uma singularidade $A_{2}$. Se $a_{2}=a_{6}=0$, o ponto $(0: 0: 1: 0)$ 
ocorre como ponto singular não isolado.

(c) Seja $x_{3}=1$, então obtemos

$$
F\left(x_{0}, x_{1}, x_{2}, 1\right)=x_{0} x_{1}+x_{0} a\left(x_{0}, x_{2}\right)+x_{1} b\left(x_{1}, x_{2}\right)+a_{6} x_{2}^{3} .
$$

Se $f_{3}(0,0,1) \neq 0 \Rightarrow a_{6} \neq 0 P$ é uma singularidade $A_{2}$. Caso contrário, suponhamos que $x_{0}=0$ encontre $f_{3}$ uma vez em $(0: 0: 1)$, então temos $k_{0}=1, a_{6}=0$ e $a_{2} \neq 0$. Por uma mudança de coordenadas temos que $a\left(x_{0}, x_{2}\right)=x_{2}\left(x_{0}+a_{2} x_{2}\right)$ e seja $x_{1}^{\prime}=x_{1}+x_{2}\left(x_{0}+a_{2} x_{2}\right)$ assim obtemos $x_{0} x_{1}^{\prime}+\left[x_{1}^{\prime}-x_{2}\left(x_{0}+a_{2} x_{2}\right)\right]\left\{a_{3}\left[x_{1}^{\prime}-x_{2}\left(x_{0}+a_{2} x_{2}\right)\right]^{2}+a_{4}\left[x_{1}^{\prime}-x_{2}\left(x_{0}+a_{2} x_{2}\right)\right] x_{2}+\right.$ $\left.a_{5} x_{2}^{2}\right\}$.

Usando pesos $(k, k, 2)$ para $k=4,5,6$, temos uma singularidade $A_{k-1}$ em $P$, se e somente se, $a_{5}=\ldots=a_{10-k}, a_{9-k} \neq 0$, como queríamos.

\subsubsection{Descrevendo a classificação}

Aplicando o lema acima para várias configurações da cúbica e o par de retas obtemos a seguinte lista. Aqui novamente cada tipo sem parâmetro é equivalente a uma forma normal.

Notação: $a^{b} \cdot c^{d}$ : significa que $f_{3}=0$ tem $b$ interseções com $x_{0}=0$ com multiplicidades $a$, e $d$ interseções com $x_{1}=0$ com multiplicidades $c$ fora de $(0: 0: 1)$.

\begin{tabular}{|l|l|l|l|l|l|l|l|l|l|l|l|l|l|}
\hline partição & $1^{3} .1^{3}$ & $1^{3} .21$ & $1^{3} .3$ & 21.21 & 21.3 & 3.3 & $1^{2} .1^{2}$ & $1^{2} .2$ & 2.2 & $1^{2} .1$ & 2.1 & $1^{2} .0$ & 2.0 \\
\hline singularidade & $A_{2}$ & $A_{2} A_{1}$ & $2 A_{2}$ & $A_{2} 2 A$ & $2 A_{2} A_{1}$ & $3 A_{2}$ & $A_{3}$ & $A_{3} A_{1}$ & $A_{3} 2 A_{1}$ & $A_{4}$ & $A_{4} A_{1}$ & $A_{5}$ & $A_{5} A_{1}$ \\
\hline $\begin{array}{l}\mathrm{N}^{\circ} \text { de } \\
\text { Parâmetros }\end{array}$ & 2 & 1 & 1 & 0 & 0 & 0 & 1 & 0 & 0 & 0 & 0 & 0 & 0 \\
\hline
\end{tabular}

Tabela 5.2: $f_{2}$ é binó

INTERPRETANDO A TABELA 
Para interpretar a tabela vamos dividir em 4 etapas considerando o tipo de singularidade de $P$.

Primeiro vamos supor que $P$ é uma singularidade $A_{2}$, nesse caso temos as seguintes possibilidades:

$1^{3} \cdot 1^{3} \rightarrow f_{3}=0$ possui três interseções simples com $x_{0}=0$ e três interseções simples com $x_{1}=0$, isto é, $F$ não tem singularidade no infinito, a única singularidade é $P$, logo temos uma singularidade $A_{2}$. Aqui o número de parâmetros é 2 .

$1^{3} .21 \rightarrow f_{3}=0$ possui três interseções simples com $x_{0}=0$, uma interseção simples e uma dupla com $x_{1}=0$, isto é, $F$ tem uma singularidade no infinito com $x_{1}=0$, digamos $Q=(1: 0: 0: 0), P$ é uma singularidade $A_{2}$ e $Q$ uma sigularidade $A_{1}$. O número de parâmetros neste caso é 1 .

$1^{3} .3 \rightarrow f_{3}=0$ possui três interseções simples com $x_{0}=0$, uma interseção tripla com $x_{1}=0$, isto é, $F$ tem uma singularidade no infinito com $x_{1}=0$, digamos em $Q=(1: 0: 0: 0)$,os pontos $P$ e $Q$ são singularidades $A_{2}$ e o número de parâmetros é 1 .

$21.21 \rightarrow f_{3}=0$ possui uma interseção simples e uma dupla com $x_{0}=0$, uma interseção simples e uma dupla com $x_{1}=0$, isto é, $F$ tem duas singularidades no infinito, uma com $x_{1}=0$ e outra com $x_{0}=0$, digamos $Q=(1: 0: 0: 0), R=(0: 1: 0: 0), P$ é uma singularidade $A_{2}, Q$ e $R$ sigularidades $A_{1}$. Neste caso não há parâmetros.

$21.3 \rightarrow f_{3}=0$ possui uma interseção simples e uma dupla com $x_{0}=0$ e uma interseção tripla com $x_{1}=0$, isto é, $F$ tem duas singularidades no infinito, uma com $x_{1}=0$ e outra com $x_{0}=0$, digamos $Q=(1: 0: 0: 0)$ 
e $R=(0: 1: 0: 0)$. Neste caso $P$ e $Q$ são singularidades $A_{2}$ e $R$ uma sigularidade $A_{1}$. Aqui não há parâmetros.

$3.3 \rightarrow f_{3}=0$ possui uma interseção tripla com $x_{0}=0$ e uma interseção tripla com $x_{1}=0$, isto é, $F$ tem duas singularidades no infinito, uma com $x_{1}=0$ e outra com $x_{0}=0$, digamos $Q=(1: 0: 0: 0)$ e $R=(0: 1: 0: 0)$. Os pontos $P, Q$ e $R$ são singularidades $A_{2}$. Neste caso não há parâmetros.

Agora vamos considerar o caso em que $P$ é uma singularidade $A_{3}$, nesse caso temos as seguintes possibilidades:

$1^{2} .1^{2} \rightarrow f_{3}=0$ possui duas interseções simples com $x_{0}=0$ e duas interseções simples com $x_{1}=0$, isto é, $F$ não tem singularidade no infinito, a única singularidade é $P$ que é uma singularidade $A_{3}$. Aqui o número de parâmetro é 1 .

$1^{2} .2 \rightarrow f_{3}=0$ possui duas interseções simples com $x_{0}=0$ e uma interseção dupla com $x_{1}=0$, isto é, $F$ tem uma singularidade no infinito,com $x_{1}=0$, digamos $Q=(1: 0: 0: 0)$. Neste caso, $P$ é uma singularidade $A_{3} \mathrm{e}$ $Q$ uma singularidade $A_{1}$, e não há parâmetro.

$2.2 \rightarrow f_{3}=0$ possui uma interseção dupla com $x_{0}=0$ e uma interseção dupla com $x_{1}=0$, isto é, $F$ tem duas singularidades no infinito, sendo uma com $x_{1}=0$ e outra com $x_{0}=0$, digamos $Q=(1: 0: 0: 0)$ e $R=(0: 1: 0: 0)$. Aqui $P$ é uma singularidade $A_{3}, Q$ e $R$ são singularidades $A_{1}$. Neste caso não há parâmetro.

Considerando o caso em que $P$ é uma singularidade $A_{4}$, temos as seguintes possibilidades: 
$1^{2} .1 \rightarrow f_{3}=0$ possui duas interseções simples com $x_{0}=0$ e uma interseção simples com $x_{1}=0$, isto é, $F$ não tem singularidades no infinito, a única singularidade é $P$ que é uma singularidade $A_{4}$. Não há parâmetro neste caso.

$2.1 \rightarrow f_{3}=0$ possui uma inteseção dupla com $x_{0}=0$ e uma interseção simples com $x_{1}=0$, isto é, $F$ tem uma singularidade no infinito, com $x_{0}=0$, digamos $Q=(1: 0: 0: 0)$,que é uma singularidade $A_{1}$. Neste caso não há parâmetro.

Considerando o caso em que $P$ é uma singularidade $A_{5}$, temos as seguintes possibilidades:

$1^{2} .0 \rightarrow f_{3}=0$ possui duas interseções simples com $x_{0}=0$ e não intersecta com $x_{1}=0$, isto é, $F$ não tem singularidade no infinito, a única singularidade é $P$ que é uma singularidade $A_{5}$. Neste caso não há parâmetro.

$2.0 \rightarrow f_{3}=0$ possui uma interseção dupla com $x_{0}=0$ e não intersecta com $x_{1}=0$, isto é, $F$ tem uma singularidade no infinito,com $x_{0}=0$, digamos $Q=(1: 0: 0: 0)$, que é uma singularidade $A_{1}$. Neste caso não há parâmetro.

Note que a interseção $\left\{f_{2}=0\right\} \cap\left\{f_{3}=0\right\}$ é dada pelas retas $\left\{x_{0}=\right.$ $0\} \cap\left\{f_{3}=0\right\}$ e $\left\{x_{1}=0\right\} \cap\left\{f_{3}=0\right\}$. Podemos ter no máximo duas singularidades (distintas) no infinito.

Se $F$ tem uma única singularidade no infinito, podemos assumir sem perda de generalidade que o ponto é $Q=(1: 0: 0: 0)$. Se $F$ tem duas singularidades no infinito, podemos assumir sem perda de generalidade que os pontos são $Q=(1: 0: 0: 0)$ e $R=(0: 1: 0: 0)$. 
Notação: Seja $\mathcal{R}_{1}$ denotando a resultante de $f_{3}\left(0, x_{1}, x_{2}\right)$ com sua respectiva derivada, e $\mathcal{R}_{2}$ a resultante $f_{3}\left(x_{0}, 0, x_{2}\right)$ com sua respectiva derivada. Proposição 5.13. (a) Se $F$ é regular no infinito, então $\mathcal{R}_{1} \neq 0$ e $\mathcal{R}_{2} \neq 0$.

(b) Se F tem uma única singularidade no infinito, então $\mathcal{R}_{1} \neq 0$ e $\mathcal{R}_{2}=0$.

(c) Se F tem duas singularidades no infinito, então $\mathcal{R}_{1}=0$ e $\mathcal{R}_{2}=0$.

\subsubsection{Refinamento da classificação quando $f_{2}$ é binó}

Nas próximas proposições apresentamos um refinamento da classificação anterior, em termos da classificação das singularidades da $f_{3}$.

Como $P$ é um binó, podemos tomar $F$ da seguinte forma:

$F=x_{3} x_{0} x_{1}+x_{0}\left(a_{0} x_{0}^{2}+a_{1} x_{0} x_{2}+a_{2} x_{2}^{2}\right)+x_{1}\left(a_{3} x_{1}^{2}+a_{4} x_{1} x_{2}+a_{5} x_{2}^{2}\right)+a_{6} x_{2}^{3}=0$.

Neste caso, sabemos que $P$ é de corank 1, isto é, uma singularidade do tipo $A_{k}, k \geq 2$, ou seja, $P$ é uma singularidade do tipo $A_{k}, \quad k=1,2,3,4$ ou 5. Vamos dividir em itens através do tipo da singularidade em $P$ :

Nota 5.14. Vamos analisar as singularidades fora do ponto singular $(0: 0$ : 1).

1) $P$ é uma singularidade de tipo $A_{2}$, neste caso então $a_{6} \neq 0$.

Nota 5.15. Se F é de tipo $1^{3} \cdot 1^{3}$, então $F$ é regular no infinito.

Consideramos primeiramente o caso em que o ponto $Q=(1: 0:: 0: 0)$ é a única singularidade no infinito de $F$. 
Proposição 5.16. Se F tem singularidade de tipo $1^{3} .21$ temos que:

a) $f_{3}$ é uma cúspide, se e somente se, $a_{3} \neq 0$.

b) $f_{3}$ é cônica mais tangente, se e somente se, $a_{3}=0$.

Demonstração. De fato, como $Q=(1: 0: 0: 0)$ é a única singularidade no infinito temos que $a_{0}=a_{1}=0$ e $a_{4} \neq 0$. Além disso, como $Q$ é uma singularidade de tipo $A_{1}$, temos que $a_{2} \neq 0$. Assim temos que se $a_{3} \neq 0$, a forma cúbica $f_{3}$ é uma cúspide e se $a_{3}=0 f_{3}$ é cônica mais tangente.

Proposição 5.17. Temos que F é de tipo $1^{3} .3$, se e somente se, $f_{3}$ são três retas concorrentes.

Demonstração. Como $Q=(1: 0: 0: 0)$ é a única singularidade no infinito temos que $a_{0}=a_{1}=0$ e Res $\left(f_{3}, \frac{\partial f_{3}}{\partial x_{2}}\right) \neq 0$. Além disso como $Q$ é uma singularidade de tipo $A_{2}$, temos que $a_{2}=0, \operatorname{logo} f_{3}$ são três retas concorrentes.

Agora vamos considerar o caso onde $F$ tem duas singularidades no infinito, $Q=(1: 0: 0: 0)$ e $R=(0: 1: 0: 0)$.

Proposição 5.18. O polinômio $F$ é de tipo 21.21, se e somente se, $f_{3}$ é uma reta dupla mais uma reta simples.

Demonstração. Como $Q=(1: 0: 0: 0)$ e $R=(0: 1: 0: 0)$ são pontos singulares no infinito, então $a_{0}=a_{1}=a_{3}=a_{4}=0$ e como ambos são singularidades de tipo $A_{1}$, temos que $a_{2} \cdot a_{5} \neq 0$, assim, a forma cúbica $f_{3}$ é reta dupla mais uma reta simples.

Proposição 5.19. A cúbica $F$ é de tipo 21.3, se e somente se, $f_{3}$ é uma reta dupla mais uma reta simples.

Demonstração. Como $Q=(1: 0: 0: 0)$ e $R=(0: 1: 0: 0)$ são pontos singulares no infinito, temos que $a_{0}=a_{1}=a_{3}=a_{4}=0$. Como $Q$ é uma 
singularidade de tipo $A_{2}$, temos que $a_{2}=0$ e sendo $R$ uma singularidade de tipo $A_{1}$ tem-se que $a_{5} \neq 0$. Assim temos que a forma cúbica $f_{3}$ é uma reta dupla mais uma reta simples.

Proposição 5.20. A cúbica $F$ é de tipo 3.3 , se e somente se, $f_{3}$ é uma reta tripla.

Demonstração. Novamente como $Q=(1: 0: 0: 0)$ e $R=(0: 1: 0: 0)$ são pontos singulares no infinito, temos que $a_{0}=a_{1}=a_{3}=a_{4}=0$. Como ambos os pontos $Q$ e $R$ são singularidades de tipo $A_{2}$, segue que $a_{2}=a_{5}=0$. Assim, a forma cúbica $f_{3}$ é uma reta reta tripla.

2) $P$ é uma singularidade do tipo $A_{3}$, neste caso, então $a_{6}=0$ e $a_{2} \cdot a_{5} \neq 0$.

Nota 5.21. Se F é de tipo $1^{2} \cdot 1^{2}$, então $F$ é regular no infinito.

Consideramos primeiramente o caso em que $F$ tem uma única singularidade no infinito, $Q=(1: 0: 0: 0)$.

Proposição 5.22. Se F tem singularidade de tipo $1^{2} .2$ temos que:

a) $f_{3}$ é uma cúspide, se e somente se, $a_{3} \neq 0$.

b) $f_{3}$ é cônica mais tangente, se e somente se, $x_{3}=0$.

Demonstração. Já que $Q$ é a única singularidade no infinito, temos que $a_{0}=a_{1}=0$ e $a_{3} \cdot a_{5}-\frac{a_{4}^{2}}{4} \neq 0$. Se $a_{3} \neq 0$, a forma cúbica $f_{3}$ é cúspide e se $a_{3}=0$ temos que $f_{3}$ é cônica mais tangente.

Observamos que $f_{3}$ não pode ser uma reta tripla, pois $a_{5} \neq 0$.

2) Agora vamos considerar o caso em que $F$ tem duas singularidades no infinito. 
Proposição 5.23. Temos que $F$ é da forma 2.2, se e somente se, $f_{3}$ é uma reta dupla mais uma reta simples.

Demonstração. Como $Q$ e $R$ são pontos singulares no infinito, temos que $a_{0}=a_{1}=a_{3}=a_{4}=0$ e $a_{2} \neq 0$. Neste caso segue que forma cúbica $f_{3}$ é uma reta dupla mais uma reta simples.

3) $P$ é uma singularidade do tipo $A_{4}$, neste caso então $a_{6}=0, a_{2}=0$ ou $a_{5}=0$ e $a_{2} \cdot a_{4} \neq 0$.

Nota 5.24. Se $F$ é de tipo $1^{2} .1$, então $F$ é regular no infinito.

Proposição 5.25. Se F é do tipo 1.2, então $f_{3}$ pode ser das seguintes formas:

a) Cúspide, se $a_{3} \neq 0$.

b) Cônica mais tangente, se $a_{3}=0$.

Demonstração. Como $Q$ é o único ponto singular no infinito temos que $a_{0}=a_{1}=0$. Como $a_{2} \neq 0$ ou $a_{5} \neq 0$, se $a_{3} \neq 0$ temos que a forma Cúbica de $f_{3}$ é uma cúspide e se $a_{3}=0$ então $f_{3}$ é cônica mais tangente.

4) $P$ é uma singularidade do tipo $A_{5}$, neste caso $a_{6}=a_{5}=a_{4}=0$ e $a_{3} \neq 0$.

Nota 5.26. Se F é de tipo $1^{2} .0$, então $F$ é regular no infinito.

Proposição 5.27. Temos que $F$ é do tipo 0.2 , se e somente se, f $f_{3}$ é uma cúspide.

Demonstração. Como $Q$ é o único ponto singular no infinito temos que $a_{0}=a_{1}=0$ e como a singularidade é de tipo $A_{1}$ temos que $a_{2} \neq 0$. $\log f_{3}$ é uma cúspide.

A próxima tabela é um resumo dos resultados descritos nas proposições anteriores. 


\begin{tabular}{|c|c|c|}
\hline S. de interseção & $f_{3}$ & condições \\
\hline $1^{3} \cdot 1^{3}$ & regular & $a_{0} \neq 0, a_{1} \neq 0, a_{3} \neq 0$ e $a_{4} \neq 0$ \\
\hline $1^{3} .21$ & cúspide & $a_{2} \neq 0$ e $a_{3} \neq 0$ \\
\hline $1^{3} .21$ & cônica mais tangente & $a_{2} \neq 0$ e $a_{3}=0$ \\
\hline $1^{3} .3$ & três retas concorrentes & $a_{2}=0$ \\
\hline 21.21 & reta dupla mais reta simples & $a_{2} . a_{5} \neq 0$ \\
\hline 21.3 & reta dupla mais reta simples & $a_{2}=0$ e $a_{5} \neq 0$ \\
\hline 3.3 & reta tripla & $a_{2}=a_{5}=0$ \\
\hline $1^{2} .1^{2}$ & regular & $a_{0} \neq 0, a_{1} \neq 0, a_{3} \neq 0$ e $a_{4} \neq 0$ \\
\hline $1^{2} .2$ & cúspide & $a_{3} \neq 0$ e $a_{3} a_{5}-\frac{a_{4}^{2}}{4} \neq 0$ \\
\hline $1^{2} .2$ & cônica mais tangente & $a_{3}=0$ e $a_{3} a_{5}-\frac{a_{4}^{2}}{4} \neq 0$ \\
\hline 2.2 & reta dupla mais reta simples & $a_{2} . a_{5} \neq 0$ \\
\hline $1^{2} .1^{2}$ & regular & $a_{0} \neq 0, a_{1} \neq 0, a_{3} \neq 0$ e $a_{4} \neq 0$ \\
\hline 1.2 & cúspide & $a_{2} \neq 0$ e $a_{3} \neq 0$ \\
\hline 1.2 & cônica mais tangente & $a_{2} \neq 0$ e $a_{3}=0$ \\
\hline $1^{2}, 0$ & regular & $a_{0} \neq 0, a_{1} \neq 0, a_{3} \neq 0$ e $a_{4} \neq 0$ \\
\hline 0.2 & cúspide & $a_{2} \neq 0$ \\
\hline
\end{tabular}

Tabela 5.3: Forma normal da $f_{3}$, quando $f_{2}$ é binó

\section{5 $P$ é nó degenerado}

Como $P$ é nó degenerado, então podemos tomar $f_{2}=x_{0}^{2}$.

Lema 5.28. (Lema 4, [11]) Seja $F=x_{3} x_{0}^{2}+f_{3}\left(x_{0}, x_{1}, x_{2}\right)$. Se $x_{0}=0$ corta $f_{3}=0$ em 3 pontos distintos, 1 ponto duplo e 1 ponto simples ou em 1 ponto triplo, então $F=0$ tem uma singularidade do tipo $D_{4}, D_{5}$ ou $E_{6}$ respectivamente em $(0: 0: 0: 1)$ e nem um outro. F também tem duas possiveis formas para o caso $D_{4}$ e uma única forma para $D_{5}$ e $E_{6}$.

Demonstração. Podemos escrever $F=x_{3} x_{0}^{2}+x_{0} g_{2}\left(x_{1}, x_{2}\right)+g_{3}\left(x_{1}, x_{2}\right)$, onde $g_{i}$ é homogêneo de grau $i$. Para os três casos acima podemos tomar $g_{3}$ como $x_{1}^{3}+x_{2}^{3}, x_{1}^{2} x_{2}$ ou $x_{1}^{3}$, e fazendo $x_{3}=1$ obtemos $x_{0}^{2}+x_{0} g_{2}\left(x_{1}, x_{2}\right)+$ $g_{3}\left(x_{1}, x_{2}\right)$. Assumimos os pesos $(3,2,2),(4,3,2)$ e $(6,4,3)$ respectivamente 
para $\left(x_{0}, x_{1}, x_{2}\right)$, podemos verificar que as singularidades são do tipo $D_{4}, D_{5}$ e $E_{6}$ respectivamente em $(0: 0: 0: 1)$ provando nos dois últimos casos que $g_{2}(0,1) \neq 0$. Mas se $x_{0}=x_{1}=0$, a singularidade é uma reta dupla. Para $D_{5}, F=x_{3} x_{0}^{2}+x_{0}\left(a x_{1}^{2}+b x_{1} x_{2}+c x_{2}^{2}\right)+x_{1}^{2} x_{2}$, e por uma mudança de coordenadas adequada podemos supor que $a=b=0$. Já que temos somente singularidades isoladas $c \neq 0$ e reduzimos a forma normal para $x_{3} x_{0}^{2}+x_{0} x_{2}^{2}+x_{1}^{2} x_{2}$. É fácil verificar que não existe mais singularidades. Similarmente para $E_{6}$, onde reduzimos para a forma normal $x_{3} x_{0}^{2}+x_{0} x_{2}^{2}+x_{1}^{3}$. Para superfícies com singularidade do tipo $D_{4}$ não é difícil reduzir para uma das seguintes formas
(a) $x_{3} x_{0}^{2}+x_{1}^{3}+x_{2}^{3}+x_{0} x_{1} x_{2}$
(b) $x_{3} x_{0}^{2}+x_{1}^{3}+x_{2}^{3}$.

Novamente não podemos ter mais singularidades. Além disso (a) e (b) definem superfícies distintas projetivamente. Por exemplo a Hessiana de (a) é $x_{0}^{2}\left(x_{0}^{2}+36 x_{1} x_{2}\right)=0$, enquanto que a de (b) é $x_{0}^{2} x_{1} x_{2}=0$, as quais são claramente distintas. Alternativamente (b) tem ponto Eckardt em (1:0:0:0), isto é, um ponto simples, onde o lugar tangente corta a superfície em três retas concorrentes, enquanto em (a) não possui tal ponto.

\subsubsection{Refinamento da classificação quando $f_{2}$ é nó de- generado}

Na próxima proposição apresentamos um refinamento da classificação anterior, em termos da classificação das singularidades da $f_{3}$.

Pelo Lema 5.28 podemos tomar $F=x_{3} x_{0}^{2}+x_{0} g_{2}\left(x_{1}, x_{2}\right)+g_{3}\left(x_{1}, x_{2}\right)$, em que $g_{3}$ pode ser escrita da seguinte forma $x_{1}^{3}+x_{2}^{3}, x_{1}^{2} x_{2}$ ou $x_{1}^{3}$. 
Vamos considerar $f_{3}$ sendo a parte homogênea de grau 3 da $F$.

Proposição 5.29. Seja $F=x_{3} x_{0}^{2}+x_{0} g_{2}\left(x_{1}, x_{2}\right)+g_{3}\left(x_{1}, x_{2}\right)$, onde $g_{3}$ é $x_{1}^{3}+x_{2}^{3}, x_{1}^{2} x_{2}$ ou $x_{1}^{3}$. Então $f_{3}$ pode assumir uma das seguintes formas: nodal, cúspide, cônica mais tangente, cônica mais corda, três retas concorrentes, triângulo, reta dupla mais reta simples ou reta tripla.

Demonstração. Seja $F=x_{3} x_{0}^{2}+x_{0} g_{2}\left(x_{1}, x_{2}\right)+g_{3}\left(x_{1}, x_{2}\right)$.

$1^{0}$ caso: Primeiro vamos considerar o caso onde $g_{3}$ tem 3 raízes distintas. Neste caso podemos considerar que $g_{3}$ são 3 retas concorrentes e $g_{3} \approx x_{1}^{3}+x_{2}^{3}$.

Observe que se $g_{2} \equiv 0, f_{3}$ são 3 retas concorrentes. Vamos considerar $g_{2} \not \equiv 0$.

(a) Primeiro vamos analisar o caso $\delta\left(g_{2}\right) \neq 0$.

(i) Res $\left(g_{2}, g_{3}\right) \neq 0$. Temos que $f_{3}$ pode ser escrita da seguinte forma $f_{3}=x_{0}\left(a_{1} x_{1}-a_{2} x_{2}\right)\left(b_{1} x_{1}-b_{2} x_{2}\right)+\left(\alpha_{1} x_{1}-\alpha_{2} x_{2}\right)\left(\beta_{1} x_{1}-\beta_{2} x_{2}\right)\left(\gamma_{1} x_{1}-\gamma_{2} x_{2}\right)$. Já que 3 pontos de uma reta projetiva podem ser colocados em posição geral, por uma mudança de coordenadas podemos escrever $f_{3}$ da seguinte forma

$$
f_{3}=x_{0} x_{1} x_{2}+\left(x_{1}+x_{2}\right)\left(x_{1}+\alpha x_{2}\right)\left(x_{1}+\beta x_{2}\right),
$$

e neste caso temos que $f_{3}$ é nodal e $f$ é regular no infinito.

(ii) Res $\left(g_{2}, g_{3}\right)=0$. Neste caso temos duas possibilidades: $g_{2}$ e $g_{3}$ têm um único fator comum, ou $g_{2}$ e $g_{3}$ têm dois fatores comuns. Se $g_{2}$ e $g_{3}$ tem um único fator comum, por uma mudança de coordenadas, $f_{3}$ pode ser escrita como 


$$
f_{3}=x_{0} x_{1} x_{2}+x_{1}\left(x_{1}+x_{2}\right)\left(x_{1}+\alpha x_{2}\right) .
$$

Neste caso, temos que $f_{3}$ é cônica mais corda. Se $g_{2}$ e $g_{3}$ têm dois fatores comuns, por uma mudança de coordenadas $f_{3}$ pode ser escrita como

$$
f_{3}=x_{0} x_{1} x_{2}+x_{1} x_{2}\left(x_{1}+x_{2}\right) .
$$

Neste caso $f_{3}$ é um triângulo. Em ambos os casos, $f$ é regular no infinito.

(b) Agora vamos analisar o caso $\delta\left(g_{2}\right)=0$. Temos que $f_{3}$ se escreve da seguinte forma

$$
f_{3}=x_{0}\left(a_{1} x_{1}-a_{2} x_{2}\right)^{2}+\left(\alpha_{1} x_{1}-\alpha_{2} x_{2}\right)\left(\beta_{1} x_{1}-\beta_{2} x_{2}\right)\left(\gamma_{1} x_{1}-\gamma_{2} x_{2}\right) .
$$

(i) Res $\left(g_{2}, g_{3}\right) \neq 0$. Por uma mudança de coordenada $f_{3}$ pode ser escrita como

$$
f_{3}=x_{0} x_{1}^{2}+x_{2}\left(x_{1}+x_{2}\right)\left(x_{1}+\alpha_{1} x_{2}\right),
$$

neste caso temos que $f_{3}$ é uma cúspide. Temos que $f$ é regular no infinito.

(ii) Res $\left(g_{2}, g_{3}\right)=0$. Há dois casos a considerar: $g_{2}$ e $g_{3}$ têm um único fator comum, ou $g_{2}$ e $g_{3}$ têm dois fatores comuns. Se $g_{2}$ e $g_{3}$ têm um único fator comum, por uma mudança de coordenadas $f_{3}$ pode ser escrita como

$$
f_{3}=x_{0} x_{1}^{2}+x_{1} x_{2}\left(x_{1}+x_{2}\right) .
$$

Aqui temos que $f_{3}$ é cônica mais tangente. Se $g_{2}$ e $g_{3}$ têm dois fatores comuns, por uma mudança de coordenadas $f_{3}$ pode ser escrita como

$$
f_{3}=x_{0} x_{1}^{2}+x_{1}^{2} x_{2},
$$

claramente temos que $f_{3}$ é reta dupla mais reta simples. No primeiro caso, quando $g_{2}$ e $g_{3}$ tem um único fator comum, temos que $f$ é regular o infinito. Já no caso onde $g_{2}$ e $g_{3}$ tem dois fatores comuns, $f$ tem uma singularidade 
não-isolada no ponto $(0: 0: 1: 0)$.

$2^{\text {o }}$ caso: Agora vamos considerar o caso em que $g_{3}$ possui uma raiz dupla e uma raiz simples. Temos que $g_{3}$ é uma reta dupla mais uma reta simples e $g_{3} \approx x_{1}^{2} x_{2}$.

Observe que se $g_{2} \equiv 0, f_{3}$ é uma reta dupla mais uma reta simples. Vamos considerar $g_{2} \not \equiv 0$.

(a) Primeiro vamos analisar o caso $\delta\left(g_{2}\right) \neq 0$.

(i) Res $\left(g_{2}, g_{3}\right) \neq 0$. Temos que $f_{3}$ pode ser escrita da seguinte forma

$$
f_{3}=x_{0}\left(a_{1} x_{1}-a_{2} x_{2}\right)\left(b_{1} x_{1}-b_{2} x_{2}\right)+\left(\alpha_{1} x_{1}-\alpha_{2} x_{2}\right)^{2}\left(\beta_{1} x_{1}-\beta_{2} x_{2}\right) .
$$

Por uma mudança de coordenadas podemos escrever $f_{3}$ como

$$
f_{3}=x_{0} x_{1}\left(x_{1}+x_{2}\right)+x_{2}^{2}\left(x_{1}+\alpha x_{2}\right)
$$

e neste caso temos que $f_{3}$ é cúspide. $f$ é regular no infinito.

(ii) Res $\left(g_{2}, g_{3}\right)=0$. Neste caso temos duas possibilidades: $g_{2}$ e $g_{3}$ têm um único fator comum, ou $g_{2}$ e $g_{3}$ têm dois fatores comuns. Se $g_{2}$ e $g_{3}$ têm um único fator comum, por uma mudança de coordenadas, $f_{3}$ pode ser escrita como

$$
f_{3}=x_{0} x_{1} x_{2}+x_{1}^{2}\left(x_{1}+x_{2}\right) .
$$

Neste caso, temos que $f_{3}$ é cônica mais corda. Se $g_{2}$ e $g_{3}$ têm dois fatores comuns, por uma mudança de coordenadas $f_{3}$ pode ser escrita como

$$
f_{3}=x_{0} x_{1} x_{2}+x_{1}^{2} x_{2} .
$$


Neste caso $f_{3}$ é um triângulo. Em ambos os casos, $f$ têm singularidade nãoisolada no ponto $(0: 0: 1: 0)$.

(b) Agora vamos analisar o caso $\delta\left(g_{2}\right)=0$. Temos que $f_{3}$ se escreve da seguinte forma

$$
f_{3}=x_{0}\left(a_{1} x_{1}-a_{2} x_{2}\right)^{2}+\left(\alpha_{1} x_{1}-\alpha_{2} x_{2}\right)^{2}\left(\beta_{1} x_{1}-\beta_{2} x_{2}\right) .
$$

(i) Res $\left(g_{2}, g_{3}\right) \neq 0$. Por uma mudança de coordenada $f_{3}$ pode ser escrita como

$$
f_{3}=x_{0} x_{1}^{2}+x_{2}^{2}\left(x_{1}+x_{2}\right)
$$

neste caso temos que $f_{3}$ é uma cúspide e $f$ é regular no infinito.

(ii) Res $\left(g_{2}, g_{3}\right)=0$. Temos dois casos a considerar: $g_{2}$ e $g_{3}$ têm um único fator comum, ou $g_{2}$ e $g_{3}$ têm dois fatores comuns. Se $g_{2}$ e $g_{3}$ têm um único fator comum, por uma mudança de coordenadas $f_{3}$ pode ser escrita como

$$
f_{3}=x_{0} x_{1}^{2}+x_{1} x_{2}^{2}
$$

Aqui temos que $f_{3}$ é cônica mais tangente. Se $g_{2}$ e $g_{3}$ têm dois fatores comuns, por uma mudança de coordenadas $f_{3}$ pode ser escrita como

$$
f_{3}=x_{0} x_{1}^{2}+x_{1}^{2} x_{2},
$$

claramente temos que $f_{3}$ é reta dupla mais reta simples. No primeiro caso, $f$ é regular no infinito e no segundo caso que $f$ têm singularidade não-isolada em $(0: 0: 1: 0)$

$3^{\mathbf{o}}$ caso: Para finalizar vamos considerar o caso onde $g_{3}$ possui uma raiz tripla. Temos que $g_{3}$ é uma reta tripla e $g_{3} \approx x_{1}^{3}$.

Observe que se $g_{2} \equiv 0, f_{3}$ é uma reta tripla. Vamos considerar $g_{2} \not \equiv 0$. 
(a) Primeiro vamos analisar o caso $\delta\left(g_{2}\right) \neq 0$.

(i) Res $\left(g_{2}, g_{3}\right) \neq 0$. Temos que $f_{3}$ pode ser escrita da seguinte forma

$$
f_{3}=x_{0}\left(a_{1} x_{1}-a_{2} x_{2}\right)\left(b_{1} x_{1}-b_{2} x_{2}\right)+\left(\alpha_{1} x_{1}-\alpha_{2} x_{2}\right)^{3} .
$$

Por uma mudança de coordenadas podemos escrever $f_{3}$ como

$$
f_{3}=x_{0} x_{1}\left(x_{1}+x_{2}\right)+x_{2}^{3}
$$

e neste caso temos que $f_{3}$ é cúspide e que $f$ é regular no infinito.

(ii) Res $\left(g_{2}, g_{3}\right)=0$. Neste caso $g_{2}$ e $g_{3}$ podem ter apenas um fator comum. Por uma mudança de coordenadas $f_{3}$ pode ser escrita como

$$
f_{3}=x_{0} x_{1}^{2}+x_{1}^{3} .
$$

Aqui, $f_{3}$ é cônica mais corda. Neste caso $f$ tem singularidade não-isolada no ponto $(0: 0: 1: 0)$.

(b) Agora vamos analisar o caso $\delta\left(g_{2}\right)=0$. Temos que $f_{3}$ se escreve da seguinte forma

$$
f_{3}=x_{0}\left(a_{1} x_{1}-a_{2} x_{2}\right)^{2}+\left(\alpha_{1} x_{1}-\alpha_{2} x_{2}\right)^{3} .
$$

(i) Res $\left(g_{2}, g_{3}\right) \neq 0$. Por uma mudança de coordenada $f_{3}$ pode ser escrita como

$$
f_{3}=x_{0} x_{1}^{2}+x_{2}^{3}
$$

neste caso, $f_{3}$ é uma cúspide e que $f$ é regular no infinito.

(ii) Res $\left(g_{2}, g_{3}\right)=0$. Neste caso a única possibilidade é que $g_{2}$ e $g_{3}$ tenham dois fatores comuns, por uma mudança de coordenadas $f_{3}$ pode ser escrita como 


$$
f_{3}=x_{0} x_{1}^{2}+x_{1}^{3}
$$

claramente, $f_{3}$ é reta dupla mais reta simples. Neste caso $f$ tem singularidade não-isolada no ponto $(0: 0: 1: 0)$.

Resumimos os resultados da proposição anterior com as seguintes tabelas.

\begin{tabular}{|c|c|}
\hline$f_{3}$ & condições \\
\hline nodal & $\delta\left(g_{2}\right) \neq 0$ e Res $\left(g_{2}, g_{3}\right) \neq 0$ \\
\hline cúspide & $\delta\left(g_{2}\right)=0, g_{2} \neq 0$ e Res $\left(g_{2}, g_{3}\right) \neq 0$ \\
\hline cônica mais tangente & $\delta\left(g_{2}\right)=0, g_{2} \neq 0$ e Res $\left(g_{2}, g_{3}\right)=0$ (um único fator comum) \\
\hline cônica mais corda & $\delta\left(g_{2}\right) \neq 0$ e Res $\left(g_{2}, g_{3}\right)=0$ (um único fator comum) \\
\hline triângulo & $\delta\left(g_{2}\right) \neq 0$ e Res $\left(g_{2}, g_{3}\right)=0$ (dois fatores comuns) \\
\hline três retas concorrentes & $g_{2} \equiv 0$ \\
\hline reta dupla mais reta simples & $\delta\left(g_{2}\right)=0, g_{2} \neq 0$ e Res $\left(g_{2}, g_{3}\right)=0$ (dois fatores comuns) \\
\hline
\end{tabular}

Tabela 5.4: $g_{3}=x_{1}^{3}+x_{2}^{3}$ e $f_{2}=x_{0}^{2}$

\begin{tabular}{|c|c|}
\hline$f_{3}$ & condições \\
\hline cúspide & $\delta\left(g_{2}\right) \neq 0$ e Res $\left(g_{2}, g_{3}\right) \neq 0$ \\
\hline cúspide & $\delta\left(g_{2}\right)=0, g_{2} \neq 0$ e Res $\left(g_{2}, g_{3}\right) \neq 0$ \\
\hline cônica mais tangente & $\delta\left(g_{2}\right)=0, g_{2} \neq 0$ e Res $\left(g_{2}, g_{3}\right)=0$ (um único fator comum) \\
\hline cônica mais corda & $\delta\left(g_{2}\right) \neq 0$ e Res $\left(g_{2}, g_{3}\right)=0$ (um único fator comum) \\
\hline triângulo & $\delta\left(g_{2}\right) \neq 0$ e Res $\left(g_{2}, g_{3}\right)=0$ (dois fatores comuns) \\
\hline reta dupla mais reta simples & $\delta\left(g_{2}\right)=0, g_{2} \neq 0$ e Res $\left(g_{2}, g_{3}\right)=0$ (dois fatores comuns) \\
\hline reta dupla mais reta simples & $g_{2} \equiv 0$ \\
\hline
\end{tabular}

Tabela 5.5: $g_{3}=x_{1}^{2} x_{2}, f_{2}=x_{0}^{2}$ 


\begin{tabular}{|c|c|}
\hline$f_{3}$ & condições \\
\hline cúspide & $\delta\left(g_{2}\right) \neq 0$ e Res $\left(g_{2}, g_{3}\right) \neq 0$ \\
\hline cúspide & $\delta\left(g_{2}\right)=0, g_{2} \neq 0$ e Res $\left(g_{2}, g_{3}\right) \neq 0$ \\
\hline cônica mais corda & $\delta\left(g_{2}\right) \neq 0$ e Res $\left(g_{2}, g_{3}\right)=0$ \\
\hline reta dupla mais reta simples & $\delta\left(g_{2}\right)=0, g_{2} \neq 0$ e Res $\left(g_{2}, g_{3}\right)=0$ \\
\hline reta tripla & $g_{2} \equiv 0$ \\
\hline
\end{tabular}

Tabela 5.6: $g_{3}=x_{1}^{3}, f_{2}=x_{0}^{2}$

CASO D: $P$ é um ponto triplo.

Neste caso $f_{2}=0$ e se $F=0$ tem singularidade isolada, então $f_{3}\left(x_{1}, x_{2}, x_{3}\right)=$ 0 deve definir uma curva não singular, e $(0: 0: 0: 1)$ é uma singularidade $\widetilde{E_{6}}$. 


\section{Capítulo 6}

\section{Defeito de Betti de polinômios de grau 3 em $\mathbb{C}^{3}$}

Neste capítulo classificamos as singularidades no infinito de $f=f_{2}+f_{3}$ usando o invariante defeito de Betti introduzido por D. Siersma e M. Tibar em [39]. As principais referências para este capítulo são [45], [21], [3], [12] e [39].

\subsection{Introdução}

Seja $f: \mathbb{C}^{n} \rightarrow \mathbb{C}$ uma função polinomial de grau $d>2$ e $n>2, f(x)=$ $f_{1}(x)+\ldots+f_{d-1}(x)+f_{d}(x)$. Sabemos que $f$ é uma fibração localmente trivial sobre $\mathbb{C}$ fora de um número finito de pontos. A fibra genérica $G$ é uma variedade de Stein, (isto é, uma variedade complexa que pode ser mergulhada biholomorficamente como um subconjunto fechado de algum espaço $\mathbb{C}^{n}$ ) e consequentemente equivalente a um CW-complexo de dimensão $n-1$. E indicaremos por $b_{n-1}(f)=b_{n-1}(G)$ o $(n-1)$-ésimo número de Betti da fibra génerica. Este número de Betti é limitado por $n$ e $d$, mais precisamente $b_{n-1}(f) \leq(d-1)^{n}$. O defeito maximal de Betti de $f$ é a diferença 
$\triangle_{n-1}(f)=(d-1)^{n}-b_{n-1}(f)$. Sendo a fibra genérica $G$ uma variedade de Stein é conhecido que ela tem homologia só na dimensão média. Assim chamaremos $\triangle_{n-1}(f)$ apenas defeito de Betti.

Seja $\mathbb{P}^{n}$ a compactificação de $\mathbb{C}^{n}$, com coordenadas afins fixadas. Como antes, usamos a notação $X_{t}=f^{-1}(t)$ para a fibra de $f$ e $\overline{X_{t}} \cap H_{\infty}$ a interseção de seu fecho em $\mathbb{P}^{n}$ com o hiperplano no infinito $H^{\infty}=\mathbb{P}^{n-1}$. Denotamos por $f_{d}$ e $f_{d-1}$ as partes homogêneas de grau $d$ e $d-1$ respectivamente de $f$. Seja $\Sigma_{d}^{\infty}=\operatorname{Sing}\left(f_{d}\right)=\left\{[x] \in \mathbb{P}^{n-1} / \frac{\partial f_{d}}{\partial x_{i}}=0, i=1, \ldots, n\right\}$. Geometricamente $\Sigma_{d}^{\infty}$ é o conjunto de pontos singulares da restrição $\overline{X_{t}} \cap H_{\infty}$ e este conjunto não depende do valor de $t \in \mathbb{C}$. Chamaremos tais pontos de pontos de tangência no infinito. Observemos que o conjunto de pontos singulares no infinito definido no Capítulo 3 , satisfaz à condição $\Sigma^{\infty}=\Sigma_{d}^{\infty} \cap\left\{f_{d-1}=0\right\}$.

Uma hipersuperfície $X$ em $\mathbb{C}^{n}$ é chamada geral no infinito se seu fecho projetivo $\bar{X}$ é não singular numa vizinhança do hiperplano no infinito e além disso $\bar{X} \pitchfork H^{\infty}$.

O polinômio $f$ é chamado geral no infinito se todas as fibras de $f$ são geral no infinito. Observe que $f$ é geral no infinito, se e somente se $\Sigma_{d}^{\infty}=\emptyset$.

\subsection{Deformações de polinômios}

Apresentamos nesta seção alguns resultados de [39] que serão necessários para nosso estudo da topologia da fibra.

Uma deformação a 1-parâmetro de $f$ é uma aplicação holomorfa $P$ : $\mathbb{C}^{n} \times \mathbb{C} \rightarrow \mathbb{C}$, em que $P_{s}=P(., s)$ é um polinômio de grau $d$ para qualquer $s \in \mathbb{C}$ e tal que $P_{0}=f$. Vamos trabalhar com germes em $s=0$ de tais 
famílias de polinômios. Seja $G_{s}$ a fibra genérica de $P_{s}$.

Proposição 6.1. (Prop. 2.1, [39]) Para $s \neq 0$ suficientemente pequeno, a fibra especial $G_{0}$ de $P_{0}$ pode ser naturalmente mergulhada na fibra genérica $G_{s}$ de $P_{s}$ tal que o mergulho $G_{0} \subset G_{s}$ induz uma aplicação injetiva nos grupos de homologia $H_{n-1}\left(G_{0}\right) \hookrightarrow H_{n-1}\left(G_{s}\right)$.

Proposição 6.2. (Prop. 2.2 [39]) Qualquer polinômio pode ser deformado em um polinômio geral no infinito de mesmo grau. Mais precisamente seja $h_{d}$ um polinômio homogêneo de grau d geral no infinito. Então a deformação $f_{\epsilon}=f+\epsilon h_{d}$ transforma qualquer polinômio $f$ de grau $d$ em um polinômio $f_{\epsilon}$ geral no infinito, para todo $\epsilon \neq 0$ suficientemente pequeno.

Demonstração. Vamos primeiramente observar que podemos deformar qualquer hipersuperfície $X \subset \mathbb{C}^{n}$ em uma família de grau constante $\left(X_{s}\right)_{s \in \delta}$ tal que $X=X_{0}$ e que $X_{s}$ é geral no infinito e não singular para $s \neq 0$ em um pequeno disco $\delta$ centrado na origem $0 \in \mathbb{C}^{n}$. Seja $X_{s}=\left\{(1-s) f+s\left(h_{d}-1\right)=0\right\}$. A família $\left\{X_{s}=0\right\}_{s \in[0,1]}$ tem um número finito de fibras especiais pois contém a hipersuperfície geral no infinito não singular $\left\{h_{d}-1=0\right\}$ e as propriedades de transversalidade e regularidade são abertas. Segue que somente um número finito de hipersuperfíces desta família é singular no infinito. Então a família $\left\{X_{s}\right\}_{s \in \delta}$ tem a propriedade desejada para o disco $\delta$ centrado em 0 . A seguir vamos considerar a deformação $f_{\epsilon}=f+\epsilon h_{d}$ de $f$, tomando $s=\frac{\epsilon}{\epsilon-1}$ na família acima e deduzimos que a fibra $f_{\epsilon}=\epsilon$ é geral no infinito. Como a fibra genérica de um polinômio está bem definida, concluímos que $f_{\epsilon}$ é um polinômio geral no infinito para qualquer $\epsilon \neq 0$ suficientemente pequeno.

Definição 6.3. Seja $Y_{t}=f^{-1}(t)$ e $p \in H^{\infty}$. O par de conjuntos $\left(\overline{Y_{t}}, \overline{Y_{t}} \cap\right.$ $\left.H^{\infty}\right)_{p}$ é chamado germe de fronteira, que é uma familia de germes dependendo de $t \in \mathbb{C}$. 
Esta família tem um tipo de singularidade constante exceto em um número finito de valores de $t$. A teoria geral de singularidades de fronteira foi estudada por Arnol'd [1].

Definição 6.4. Dizemos que o par de fronteira $\left(\overline{Y_{t}}, \overline{Y_{t}} \cap H^{\infty}\right)_{p}$ tem uma singularidade isolada se ambos $\overline{Y_{t}}$ e $\overline{Y_{t}} \cap H^{\infty}$ têm (no máximo) singularidades isoladas em $P$.

Por exemplo, as singularidades da tabela 5.3.1 são singularidades de fronteira isoladas.

A seguinte equivalência se verifica: $\mathrm{O}$ par $\left(\overline{Y_{t}}, \overline{Y_{t}} \cap H^{\infty}\right)$ tem singularidades isoladas, se e somente se, $Y_{t}$ tem singularidades isoladas e $\operatorname{dim} \Sigma_{d}^{\infty} \leq 0$. Então $\Sigma^{\infty}=\Sigma_{d}^{\infty} \cap\left\{f_{d-1}=0\right\}$ é um subconjunto de pontos de $H^{\infty}$ em que $\overline{Y_{t}}$ é singular e isto não depende do valor de $t \in \mathbb{C}$.

O próximo resultado é uma fórmula para calcular o defeito de Betti. Este resultado será utilizado nos cálculos do defeito de Betti deste capítulo, assim apresentamos também a demonstração.

Proposição 6.5. (Prop. 2.4 [39]) Sejam $f: \mathbb{C}^{n} \rightarrow \mathbb{C}$ um polinômio de grau d com singularidades isoladas e $X_{0}=\{f=0\}$. Suponha que dim $\Sigma_{d}^{\infty} \cap$ $\left\{f_{d-1}=0\right\} \leq 0$. Então

$$
\triangle_{n-1}(f)=\sum_{p \in \Sigma_{f}^{\infty} \cap\left\{f_{d-1}=0\right\}} \mu_{p}\left(\overline{X_{0}}\right)+(-1)^{n} \triangle \mathcal{X}^{\infty}
$$

onde $\triangle \mathcal{X}^{\infty}=\mathcal{X}^{n-1, d}-\mathcal{X}\left(\left\{f_{d}=0\right\}\right)$ e $\mathcal{X}^{n-1, d}=n-\frac{1}{d}\left\{1+(-1)^{n-1}(d-1)^{n}\right\}$ denota a característica de Euler de uma hipersuperfície suave $V_{g e n}^{n-1, d}$ de grau dem $\mathbb{P}^{n-1}$, e $\mu_{p}\left(\overline{X_{0}}\right)$ o número de Milnor de $\overline{X_{0}}$ no ponto singular $P$.

Em particular, se $\operatorname{dim} \Sigma_{d}^{\infty} \leq 0$, então 


$$
\triangle_{n-1}(f)=\sum_{p \in \Sigma_{d}^{\infty}}\left[\mu_{p}\left(\overline{X_{0}}\right)+\mu\left(\overline{X_{0}} \cap H^{\infty}\right)\right]
$$

Demonstração. Vamos considerar o germe de deformação de $X_{0}$ em uma família de grau constante $\left\{X_{s}\right\}_{s \in \delta}$ tal que $X_{s}$ é não singular e geral no infinito para $s \neq 0$, que existe de acordo com a Proposição 6.2. Para qualquer $s \in \delta$, pela propriedade aditiva da característica de Euler, temos a igualdade

$$
\mathcal{X}\left(X_{s}\right)=\mathcal{X}\left(\overline{X_{s}}\right)-\mathcal{X}\left(\overline{X_{s}} \cap H^{\infty}\right)
$$

Tomando a diferença temos,

$$
\mathcal{X}\left(X_{0}\right)-\mathcal{X}\left(X_{s}\right)=\mathcal{X}\left(\overline{X_{0}}\right)-\mathcal{X}\left(\overline{X_{s}}\right)-\mathcal{X}\left(\overline{X_{0}} \cap H^{\infty}\right)+\mathcal{X}\left(\overline{X_{s}} \cap H^{\infty}\right) .
$$

A família $\left\{\overline{X_{s}}\right\}_{s \in \delta}$ é em particular uma suavização da hipersuperfície $\overline{X_{0}} \mathrm{com}$ singularidade isolada e portanto o salto da característica de Euler é a soma dos números de Milnor das singularidades $\overline{X_{0}}[12]$.

$$
\mathcal{X}\left(\overline{X_{0}}\right)-\mathcal{X}\left(\overline{X_{s}}\right)=(-1)^{n} \sum_{p \in \Sigma_{f}^{\infty} \cap\left\{f_{d-1}=0\right\}} \mu_{p}\left(\overline{X_{0}}\right)
$$

Quando $\operatorname{dim} \Sigma_{d}^{\infty} \leq 0$, a família $\overline{X_{s}} \cap H^{\infty}$ é uma suavização de $\overline{X_{0}} \cap H^{\infty} \mathrm{e}$ temos uma igualdade similar

$$
\mathcal{X}\left(\overline{X_{0}} \cap H^{\infty}\right)-\mathcal{X}\left(\overline{X_{s}} \cap H^{\infty}\right)=(-1)^{n-1} \sum_{p \in \Sigma_{f}^{\infty}} \mu_{p}\left(\overline{X_{0}} \cap H^{\infty}\right)
$$

A seguir precisamos que a fibra genérica tenha homologia concentrada na dimensão máxima, o que é verdade, uma vez que o polinômio $f$ tem singularidade isolada. Portanto a fibra genérica tem o tipo de homotopia de um bouquet de $S^{n-1}$-esferas e consequentemente essa homologia é concentrada na dimensão $n-1$ ( Teorema 3.6). Isto implica que $\mathcal{X}\left(X_{0}\right)=1-(-1)^{n} b_{n-1}\left(X_{0}\right)$. Temos ainda que $\mathcal{X}\left(X_{s}\right)=1-(-1)^{n}(d-1)^{n}$ já que $X_{s}$ é geral no infinito e 
não singular. Juntando todos os resultados temos as fórmulas da proposição.

\subsection{Defeito de Betti de polinômios de grau 3 em $\mathbb{C}^{3}$}

No capítulo 5 fizemos um refinamento da classificação feita por Bruce e Wall em [11] em termos das singularidades de $f_{3}$. Vamos utilizar esses resultados para calcular o defeito de Betti para $f$, onde $f$ é um polinômio de grau $3 \mathrm{em}$ $\mathbb{C}^{3}$. Para calcular o defeito dessas funções polinomiais utilizaremos a Proposição 6.5 .

O lema a seguir será importante para o cálculo do defeito de Betti de $f$.

Lema 6.6. a) Se $f_{3}$ é nodal, então $\mathcal{X}\left(\left\{f_{3}=0\right\}\right)=1$.

b) $S e f_{3}$ é cúspide, então $\mathcal{X}\left(\left\{f_{3}=0\right\}\right)=2$.

c) $S e f_{3}$ é cônica mais tangente, então $\mathcal{X}\left(\left\{f_{3}=0\right\}\right)=3$.

d) Se $f_{3}$ é cônica mais corda, então $\mathcal{X}\left(\left\{f_{3}=0\right\}\right)=2$.

e) $S e f_{3}$ é triângulo, então $\mathcal{X}\left(\left\{f_{3}=0\right\}\right)=3$.

f) $S e f_{3}$ é três retas concorrentes, então $\mathcal{X}\left(\left\{f_{3}=0\right\}\right)=4$.

g) $S e f_{3}$ é uma reta dupla e uma reta simples, então $\mathcal{X}\left(\left\{f_{3}=0\right\}\right)=3$.

h) Se $f_{3}$ é uma reta tripla, então $\mathcal{X}\left(\left\{f_{3}=0\right\}\right)=2$.

Demonstração. Nos itens $(a)$ até $(f)$, temos que as singularidades são isoladas. Uma atenção especial será dada aos itens $(g)$ e $(h)$ em que as singularidades são não-isoladas.

a) Como $f_{3}$ é nodal, podemos supor sem perda de generalidade que $f_{3}=x_{0}^{3}+x_{1}^{3}-x_{0} x_{1} x_{2}$. A única singularidade de $f_{3}$ em $\mathbb{P}^{2}$ é $P=(0: 0: 1)$, 
que é uma singularidade do tipo $A_{1}$. Então utilizando a fórmula do salto da característica de Euler

$$
\mathcal{X}\left(\overline{X_{0}} \cap H^{\infty}\right)-\mathcal{X}\left(\overline{X_{s}} \cap H^{\infty}\right)=(-1)^{n-1} \sum_{p \in \Sigma_{f}^{\infty}} \mu_{p}\left(\overline{X_{0}} \cap H^{\infty}\right) .
$$

Temos $\mathcal{X}\left(\bar{X}_{s} \cap H^{\infty}\right)=\mathcal{X}^{2,3}=0, \operatorname{logo} \mathcal{X}\left(\bar{X}_{0} \cap H^{\infty}\right)=\mathcal{X}\left(\left\{f_{3}=0\right\}\right)=1$

Os casos $(b),(c)$ e $(f)$ que têm apenas um ponto singular no infinito seguem analogamente.

d) Como $f_{3}$ é cônica mais corda, podemos supor sem perda de generalidade que $f_{3}=x_{0}^{3}-x_{0} x_{1} x_{2}$. Temos duas singularidade de $f_{3}$ em $\mathbb{P}^{2}$ que são: $P=(0: 0: 1)$ e $Q=(0: 1: 0)$, ambas singularidades do tipo $A_{1}$. Então utilizando a fórmula do salto da característica de Euler temos que $\mathcal{X}\left(\left\{f_{3}=0\right\}\right)=2$.

O caso (e) segue analogamente.

Para provar os itens $(g)$ e $(h)$ podemos usar os resultados de [39] para o caso $\operatorname{dim} \Sigma_{f}^{\infty}>0$. Entretanto como os casos são simples, podemos calcular a característica de Euler diretamente.

g) Como $f_{3}$ é uma reta dupla e uma reta simples, $f_{3}$ é projetivamente equivalente a $f_{3}=x_{0} x_{2}^{2}$ e

$$
V\left(f_{3}\right)=\left\{\left(0: x_{1}: x_{2}\right)\right\} \cup\left\{\left(x_{0}: x_{1}: 0\right)\right\}=\mathbb{P}^{1}(\mathbb{C}) \cup_{P} \mathbb{P}^{1}(\mathbb{C}),
$$

união de duas retas projetivas com um ponto em comum $P=(0: 1: 0)$, logo $\mathcal{X}\left(\left\{f_{3}=0\right\}\right)=3$ 
h) Como $f_{3}$ é uma reta tripla, $f_{3}$ é projetivamente equivalente a $x_{0}^{3}$, e assim

$$
V\left(f_{3}\right)=\left\{\left(0: x_{1}: x_{2}\right)\right\}=\mathbb{P}^{1}(\mathbb{C}),
$$

então $\mathcal{X}\left(\left\{f_{3}=0\right\}\right)=2$.

Usando o Lema 6.3 e os resultados dos capítulos anteriores, obtemos as seguintes tabelas do defeito de Betti de $F$, onde $F=x_{3} f_{2}\left(x_{0}, x_{1}, x_{2}\right)+$ $f_{3}\left(x_{0}, x_{1} x_{3}\right)$ e $f_{2}$ é um nó cônico, binó ou nó degenerado respectivamente.

Nas tabelas seguintes, a primeira coluna representa o tipo da cúbica $f_{3}=0$ e a primeira linha o símbolo das raízes comuns de $f_{2}=f_{3}=0$.

Primeiro consideramos o caso em que $f_{2}$ é um nó cônico, e obtemos a Tabela 6.1 relacionada ao defeito de Betti $\triangle_{2}(f)$.

\begin{tabular}{|c|c|c|c|c|c|c|c|c|c|c|c|}
\hline & $1^{6}$ & $21^{4}$ & $31^{3}$ & $2^{2} 1^{2}$ & $41^{2}$ & 321 & $2^{3}$ & 51 & 42 & 33 & 6 \\
\hline Regular & 0 & - & - & - & - & - & - & - & - & - & - \\
\hline Nodal & - & 2 & 3 & - & 4 & - & - & 5 & - & - & 6 \\
\hline Cúspide & - & 3 & 4 & - & - & - & - & - & - & - & - \\
\hline Cônica mais tangente & - & 4 & 5 & - & 6 & - & - & 7 & - & - & 8 \\
\hline Cônica mais corda & - & - & - & 4 & - & 5 & - & - & 6 & 6 & - \\
\hline 3 Retas concorrentes & - & - & 5 & - & 6 & - & - & - & - & - & - \\
\hline Triângulo & - & - & - & - & - & - & 6 & - & - & - & - \\
\hline
\end{tabular}

Tabela 6.1: defeito de Betti quando $f_{2}$ é um nó cônico

Agora vamos considerar o caso em que $f_{2}$ é um binó. Neste caso, temos as Tabelas 6.2 e 6.3 relacionadas ao defeito de Betti $\triangle_{2}(f)$.

Para finalizar vamos considerar o caso em que $f_{2}$ é um nó degerado. Neste caso vimos no Lema 5.28 que $F=x_{3} x_{0}^{2}+x_{0} g_{2}\left(x_{1}, x_{2}\right)+g_{3}\left(x_{1}, x_{2}\right)$, onde $g_{i}$ é 


\begin{tabular}{|c|c|c|c|c|c|c|}
\hline & $1^{3} .1^{3}$ & $1^{3} .21$ & $1^{3} .3$ & 21.21 & 21.3 & 3.3 \\
\hline Regular & 0 & - & - & - & - & - \\
\hline Cúspide & - & 3 & - & - & - & - \\
\hline Cônica mais tangente & - & 4 & - & - & - & - \\
\hline 3 Retas Concorrentes & - & - & 5 & - & - & - \\
\hline Reta dupla e reta simples & - & - & - & 5 & 6 & - \\
\hline Reta tripla & - & - & - & - & - & 7 \\
\hline
\end{tabular}

Tabela 6.2: defeito de Betti quando $f_{2}$ é binó

\begin{tabular}{|c|c|c|c|c|c|c|c|}
\hline & $1^{2} .1^{2}$ & $1^{2} .2$ & 2.2 & $1^{2} .1$ & 1.2 & $1^{2} .0$ & 0.2 \\
\hline Regular & 0 & - & - & 0 & - & 0 & - \\
\hline Cúspide & - & 3 & - & - & 3 & - & 3 \\
\hline Cônica mais tangente & - & 4 & - & - & 4 & - & - \\
\hline Reta dupla e seta simples & - & - & 5 & - & - & - & - \\
\hline Reta tripla & - & - & - & - & - & - & - \\
\hline
\end{tabular}

Tabela 6.3: defeito de Betti quando $f_{2}$ é binó

homogêneo de grau $i$ e que $g_{3}$ pode ser escrita da seguinte forma: $x_{1}^{3}+x_{0}^{3}$, $x_{1}^{2} x_{2}$ ou $x_{1}^{3}$. A construção da Tabela 6.4 relacionada ao defeito de Betti $\triangle_{2}(f)$ neste caso será feita considerando as formas normais da $g_{3}$.

Para os resultados abaixo vamos considerar que $f\left(x_{0}, x_{1}, x_{2}\right)=f_{2}\left(x_{0}, x_{1}, x_{2}\right)+$ $f_{3}\left(x_{0}, x_{1}, x_{2}\right)$, onde $f_{2}$ é nó cônico, binó ou nó degenerado respectivamente.

Nota 6.7. (1) Nos teoremas abaixo, vamos considerar o defeito de Betti apenas nas singularidades isoladas.

(2) No caso do binó, a análise será feita fora do ponto $(0: 0: 1: 0)$.

Teorema 6.8. Seja $f: \mathbb{C}^{3} \rightarrow \mathbb{C}, f=f_{2}+f_{3}$.

Se $f$ tem uma única singularidade no infinito, então:

(a) Se $f_{3}$ é nodal, então a singularidade é do tipo $A_{k} \Leftrightarrow \triangle_{2}(f)=k+1,1 \leq$ $k \leq 5$. 


\begin{tabular}{|c|c|c|c|}
\hline$f_{3}$ & $g_{3}$ & $g_{3}$ & $g_{3}$ \\
\hline & $x_{1}^{3}+x_{2}^{3}$ & $x_{1}^{2} x_{2}$ & $x_{1}^{3}$ \\
\hline nodal & 1 & - & - \\
\hline cúspide & 2 & 2 & 2 \\
\hline cônica mais tangente & 3 & 2 & - \\
\hline cônica mais corda & 2 & 2 & $>2$ \\
\hline triângulo & 3 & $>3$ & - \\
\hline 3 reta concorrente & 4 & - & - \\
\hline reta dupla mais reta simples & $>2$ & $>2$ & $>2$ \\
\hline reta tripla & - & - & $>2$ \\
\hline
\end{tabular}

Tabela 6.4: defeito de Betti quando $f_{2}$ is nó degenerado

(b) Se $f_{3}$ é cuspidal, então a singularidade é do tipo $A_{k} \Leftrightarrow \triangle_{2}(f)=k+2,1 \leq$ $k \leq 2$.

(c) Se $f_{3}$ é cônica mais tangente, então a singularidade é do tipo $A_{k} \Leftrightarrow$ $\triangle_{2}(f)=k+3,1 \leq k \leq 5$.

(d) $\mathrm{Se}_{3}$ são três retas concorrentes, então a singularidade é do tipo $A_{k} \Leftrightarrow$ $\Leftrightarrow \triangle_{2}(f)=k+4,2 \leq k \leq 3$.

Teorema 6.9. Seja $f: \mathbb{C}^{3} \rightarrow \mathbb{C}, f=f_{2}+f_{3}$.

Se $f$ tem duas singularidades no infinito, então:

(a) $\mathrm{Se}_{3}$ é cônica mais corda, então as singularidades são dos tipos:

(i) $A_{1}+A_{1} \Leftrightarrow \triangle_{2}(f)=4$

(ii) $A_{2}+A_{1} \Leftrightarrow \triangle_{2}(f)=5$

(iii) $A_{3}+A_{1}$ ou $A_{2}+A_{2} \Leftrightarrow \triangle_{2}(f)=6$

(b) $\mathrm{Se} \mathrm{f}_{3}$ é uma reta dupla mais uma reta simples, então as singularidades são dos tipos:

(i) $A_{1}+A_{1} \Leftrightarrow \triangle_{2}(f)=5$

(ii) $A_{2}+A_{1} \Leftrightarrow \triangle_{2}(f)=6$ 
(b) Se $f_{3}$ é reta tripla, então a singularidade é do tipo $A_{2}+A_{2} \Leftrightarrow \triangle_{2}(f)=6$.

Teorema 6.10. Seja $f: \mathbb{C}^{3} \rightarrow \mathbb{C}, f=f_{2}+f_{3}$ Se $f$ tem três singularidades no infinito, então a singularidade é do tipo $A_{1}+A_{1}+A_{1} \Leftrightarrow \triangle_{2}(f)=6$. 


\section{Capítulo 7}

\section{Feixes homogêneos e classificação de funções polinomiais}

\subsection{Introdução}

Neste capítulo apresentamos uma generalização de alguns resultados de [11] para uma função polinomial da forma $f: \mathbb{C}^{n} \rightarrow \mathbb{C}$ de grau $d \geq 3$, em $n$ variáveis, $f\left(x_{0}, \ldots, x_{n}\right)=f_{d-1}\left(x_{0}, \ldots, x_{n}\right)+f_{d}\left(x_{0}, \ldots, x_{n}\right)$, em que $f_{i}$ é um polinômio homogêneo de grau $i$. O objetivo é estender para polinômios de grau mais alto, o método utilizado por Bruce e Wall, que consiste em relacionar a classificação das singularidades da homogenização de $f$ com a classificação do feixe de hipersuperfícies em $\mathbb{P}^{n}$ gerado por $f_{d-1}=0$ e $f_{d}=0$. Como aplicação dos resultados, estudamos aplicações polinomiais em $\mathbb{C}^{3}$, com apenas um ponto singular no infinito, que apresentam defeito de Betti mínimo. 


\subsection{Feixes homogêneos e classificação de aplicações polinomiais}

A seguir fixaremos algumas notações que serão usadas nos próximos resultados.

Seja $f: \mathbb{C}^{n} \rightarrow \mathbb{C}$, uma função polinomial dada por $f=f_{d_{1}}+\ldots+f_{d_{k}}$, $1 \leq d_{1}<d_{2}<\ldots<d_{k}, 1<k \leq n-1, d \geq 3$. Suponhamos que a aplicação polinomial $g: \mathbb{C}^{n} \rightarrow \mathbb{C}^{k}$ dada por $g(x)=\left(f_{d_{1}}(x), \ldots, f_{d_{k}}(x)\right)$, defina uma interseção completa $X=g^{-1}(0)$.

Lema 7.1. Se g é interseção completa com singularidade isolada (ICIS), então $\left\{\operatorname{Sing}\left(f_{d_{j}}\right)\right\} \cap\left\{g^{-1}(0)\right\} \subseteq\{0\}, \forall j=1, \ldots, k$.

Demonstração. De fato, como $g$ é ICIS, temos que $J(g)+<g>\supseteq \mathcal{M}_{n}^{l}$, para algum $l$. Isto acontece, se e somente se, $\operatorname{Sing}(g) \cap g^{-1}(0) \subseteq\{0\}$ (ver Proposição 2.24). Como $f_{d_{i}}$ são homogêneos, esta afirmação se verifica globalmente em $\mathbb{C}^{n}$. Por outro lado, $\operatorname{Sing}\left(f_{d_{j}}\right) \subseteq \operatorname{Sing}(g)$, dai segue que $\left\{\operatorname{Sing}\left(f_{d_{j}}\right)\right\} \cap\left\{g^{-1}(0)\right\} \subseteq\{0\}, \quad \forall j$.

Proposição 7.2. Sejam $f: \mathbb{C}^{n} \rightarrow \mathbb{C}$ e $g: \mathbb{C}^{n} \rightarrow \mathbb{C}^{2}$ dadas por: $f(x)=$ $f_{d-1}(x)+f_{d}(x), d \geq 3$ e $g(x)=\left(f_{d-1}(x), f_{d}(x)\right)$. Se g é ICIS então $f$ é regular no infinito.

Demonstração. Como $g$ é ICIS, temos que $\operatorname{Sing}(g) \cap g^{-1}(0) \subseteq\{0\}$. Por outro lado, como no lema anterior, temos que $\operatorname{Sing}\left(f_{d}\right) \subseteq \operatorname{Sing}(g)$. Intersectando ambos os lados desta inclusão com $\left\{f_{d}=0\right\}$ e $\left\{f_{d-1}=0\right\}$ simultaneamente temos que: 
$\left\{f_{d-1}=0\right\} \cap\left\{f_{d}=0\right\} \cap \operatorname{Sing}\left(f_{d}\right) \subseteq \operatorname{Sing}(g) \cap\left\{f_{d}=0\right\} \cap\left\{f_{d-1}=0\right\}=$ $\operatorname{Sing}(g) \cap g^{-1}(0)=\{0\}$.

Logo $f$ não tem ponto singular no infinito, ou seja, $f$ é regular no infinito.

Nota 7.3. (i) Se $f_{d-1} \equiv 0$, a hipótese da proposição se reduz à condição que $f_{d}$ tem singularidade isolada na origem e o resultado continua verdadeiro.

(ii) A recíproca da proposição acima não é verdadeira, ou seja, existem $f: \mathbb{C}^{n} \rightarrow \mathbb{C}$ e $g: \mathbb{C}^{n} \rightarrow \mathbb{C}^{2}$ tais que $f$ é regular no infinito, no entanto $g$ não é ICIS. Um exemplo é $f\left(x_{0}, x_{1}\right)=x_{0}^{4}-x_{1}^{4}+x_{0}^{3}-x_{1}^{3}$. É fácil verificar que $f$ é regular no infinito, mas $g\left(x_{0}, x_{1}\right)=\left(x_{0}^{4}-x_{1}^{4}, x_{0}^{3}-x_{1}^{3}\right)$ não é ICIS pois $\operatorname{Sing}(g) \cap g^{-1}(0)$ contém a reta $x_{0}-x_{1}=0$.

(iii) O resultado anterior não se estende para $f(x)=f_{1}(x)+\ldots+f_{k}(x)+$ $f_{d}(x)$, com $k<d-1$ e $g(x)=\left(f_{k}(x), f_{d}(x)\right)$. De fato seja $f: \mathbb{C}^{3} \rightarrow \mathbb{C}$ e g : $\mathbb{C}^{3} \rightarrow \mathbb{C}^{2}, f\left(x_{0}, x_{1}, x_{2}\right)=x_{0}^{3}+x_{1}^{3}-x_{0} x_{1} x_{2}+x_{2}$ e $g\left(x_{0}, x_{1}, x_{2}\right)=$ $\left(x_{0}^{3}+x_{1}^{3}-x_{0} x_{1} x_{2}, x_{2}\right)$ então $g$ é ICIS mas $f$ não é regular no infinito.

Corolário 7.4. Nas hipóteses da proposição anterior, se g é interseção completa e $f_{d-1} \not \equiv 0$, então as singularidades no infinito de $f$ correspondem aos pontos de tangências de $f_{d-1}^{-1}(0)$ e $f_{d}^{-1}(0)$ fora de $x=0$.

O objetivo agora é estudar a classificação de polinômios $f(x)=f_{d-1}(x)+$ $f_{d}(x)$ estendendo a abordagem de Bruce e Wall em [11]. O ponto de vista é classificar funções $f(x)=f_{d-1}(x)+f_{d}(x)$, deixando $f_{d-1}$ invariante.

Seja $F(x)$ a homogenização de $f$, então $F=0$ define uma hipersuperfície $\bar{X}$ em $\mathbb{P}^{n}$, que é o fecho projetivo de $X=f^{-1}(0)$. 
Quando $F=0$ é projetivamente equivalente à $\widetilde{F}=0 \mathrm{em} \mathbb{P}^{n}$, indicaremos por $F \sim \widetilde{F}$.

O teorema seguinte generaliza o Lema 2 de Bruce e Wall em [11].

Teorema 7.5. Sejam $f, \tilde{f}: \mathbb{C}^{n} \rightarrow \mathbb{C}$ dadas por $f(x)=f_{d-1}(x)+f_{d}(x)$, $\widetilde{f}(x)=f_{d-1}(x)+\widetilde{f}_{d}(x), d \geq 3$ e F, $\widetilde{F}$ as homogenizações de $f, \tilde{f}$ respectivamente. Indiquemos por $g, \widetilde{g}: \mathbb{C}^{n} \rightarrow \mathbb{C}^{2}$ as aplicações $g(x)=\left(f_{d-1}(x), f_{d}(x)\right)$, $\widetilde{g}(x)=\left(f_{d-1}(x), \widetilde{f}_{d}(x)\right)$ e ainda denote $X=g^{-1}(0)$ e $\widetilde{X}=\widetilde{g}^{-1}(0)$ e $P=(0$ : $\ldots: 0: 1)$. Então as seguintes condições se verificam:

(i) $F \sim \widetilde{F} \Leftrightarrow \exists l \in G l(n)$ tal que $l^{*}\left(<f_{d-1}, f_{d}>\right)=<f_{d-1}, \widetilde{f}_{d}>$.

ii) Seja $Q$ um ponto singular no infinito de $f$. Então a reta $\overline{P Q}$ está contida em $\bar{X}$. Além disso, $\left\{f_{d-1}=0\right\}$ e $\left\{f_{d}=0\right\}$ são tangentes ao longo da reta $\overline{P Q}$.

iii) Seja $S \in \bar{X}$. Se $S \in \operatorname{Sing}(\bar{X})$, então $S$ é um ponto singular no infinito de $f$ ou existe $R$ em $\overline{P S}$ com $R \in \operatorname{Sing}(f)$.

Demonstração. (i) Sejam

$$
\begin{aligned}
& F\left(x, x_{n+1}\right)=x_{n+1} f_{d-1}(x)+f_{d}(x), \\
& \widetilde{F}\left(x, x_{n+1}\right)=x_{n+1} f_{d-1}(x)+\widetilde{f}_{d}(x),
\end{aligned}
$$

e suponha que $F \sim \widetilde{F}$. Então existem uma transformação linear em $G l(n)$,

$$
\begin{gathered}
l: \mathbb{C}^{n} \rightarrow \mathbb{C}^{n} \\
x \rightarrow l(x)
\end{gathered}
$$

e 


$$
\begin{aligned}
L: \quad \mathbb{P}^{n} & \rightarrow \mathbb{P}^{n} \\
\left(x, x_{n+1}\right) & \longmapsto L\left(x, x_{n+1}\right)=\left(l(x), k\left(x, x_{n+1}\right)\right),
\end{aligned}
$$

$\operatorname{com} k\left(x, x_{n+1}\right)=a_{1} x_{1}+\ldots+a_{n} x_{n}+x_{n+1}$, tais que $F \circ L=\widetilde{F}$. Portanto $f_{d-1} \circ l=f_{d-1}$ e $k(x, 0) f_{d-1}(l(x))+f_{d}(l(x))=\widetilde{f}_{d}(x)$. Logo $l^{*}\left(<f_{d-1}, f_{d}>\right.$ )$=<f_{d-1}, \widetilde{f}_{d}>$.

Reciprocamente, se existe $l \in G l(n)$ tal que $l^{*}\left(<f_{d-1}, f_{d}>\right)=<f_{d-1}, \tilde{f}_{d}>$, então para todo $\delta \in<f_{d-1}, f_{d}>, l^{*}(\delta)=\delta \circ l \in<f_{d-1}, \widetilde{f}_{d}>$. Segue que $\exists \alpha \neq 0$, tal que $f_{d-1} \circ l=\alpha f_{d-1}$ e $f_{d} \circ l=k f_{d-1}+c \widetilde{f}_{d}$, em que $k(x)$ é uma função linear $k: \mathbb{C}^{n} \rightarrow \mathbb{C}$ e $c$ é uma constante diferente de zero. Vamos denotar

$$
f_{d}^{\prime}=k f_{d-1}+c \widetilde{f}_{d}
$$

Assim temos

$$
f=f_{d-1}+f_{d}, \quad \tilde{f}=f_{d-1}+\tilde{f}_{d} \text { e } f^{\prime}=f_{d-1}+f_{d}^{\prime} .
$$

Sejam

$$
F=x_{n+1} f_{d-1}+f_{d}, \quad \widetilde{F}=x_{n+1} f_{d-1}+\widetilde{f}_{d} \text { e } F^{\prime}=x_{n+1} f_{d-1}+f_{d}^{\prime},
$$

homogenizações de $f, \widetilde{f}_{d}$ e $f^{\prime}$ respectivamente. Note que $F \sim F^{\prime}$, pois $F\left(l, \frac{x_{n+1}}{\alpha}\right)=\frac{x_{n+1}}{\alpha} f_{d-1} \circ l+f_{d} \circ l=x_{n+1} f_{d-1}+f_{d}^{\prime}$. Agora vamos provar que $F^{\prime} \sim \widetilde{F}$. Temos que

$$
F^{\prime}\left(x, x_{n+1}\right)=x_{n+1} f_{d-1}(x)+f_{d}^{\prime}(x)=\left(x_{n+1}+k(x)\right) f_{d-1}(x)+c \widetilde{f}_{d}(x) .
$$

Assim com uma mudança linear da forma

$$
H=(I_{n}, \overbrace{x_{n+1}+k}^{X_{n+1}})
$$


obtemos $F^{\prime} \circ H=X_{n+1} f_{d-1}+c \widetilde{f}_{d}$.

Novamente por uma mudança de coordenadas lineares da forma

$$
H_{1}=\left(I_{n}, \frac{\overbrace{X_{n+1}}^{X_{n+1}^{\prime}}}{c}\right)
$$

Temos que $F^{\prime} \circ H_{1}=c\left(X_{n+1}^{\prime} f_{d-1}+\widetilde{f}_{d}\right)=c . \widetilde{F}$. Portanto $F^{\prime} \sim \widetilde{F}$.

Como $F \sim F^{\prime}$ e $F^{\prime} \sim \widetilde{F}$, temos que $F \sim \widetilde{F}$ como queríamos.

ii) Como $Q$ é ponto singular no infinito, $Q$ satisfaz ao sistema abaixo

$$
\left\{\begin{array}{r}
f_{d-1}=0 \\
\frac{\partial f_{d}}{\partial x_{j}}=0, \quad 1 \leq j \leq n
\end{array}\right.
$$

Os pontos singulares de $F=x_{n+1} f_{d-1}+f_{d}$ satisfazem ao seguinte sistema:

$$
\left\{\begin{array}{l}
\frac{\partial F}{\partial x_{j}}=x_{n+1} \frac{\partial f_{d-1}}{\partial x_{j}}+\frac{\partial f_{d}}{\partial x_{j}}=0, \quad \forall j \leq n \\
\frac{\partial F}{\partial x_{n+1}}=f_{d-1}=0
\end{array}\right.
$$

Como que $Q \in \operatorname{Sing}(F)$, então $Q \in \bar{X}$ e portanto a reta $\overline{P Q} \subset \bar{X}$. Logo, pela Prop. $7.2 f_{d-1}=0$ e $f_{d}=0$ são tangentes ao longo da reta $P Q$.

iii) Considere $\bar{X}=\left\{x_{n+1} f_{d-1}(x)+f_{d}(x)=0\right\}$ e $S=\left(s: x_{n+1}^{0}\right) \in$ $\bar{X} \cap \operatorname{Sing}(\bar{X})$. Observemos que $S$ já satisfaz as equações (1) e (2) acima. Queremos provar que $S$ é um ponto singular no infinito de $f$, ou existe $R$ na reta $\overline{P S}$ com $R \in \operatorname{Sing}(f)$.

Se $S$ é um ponto singular no infinito, então $f_{d-1}(s)=0$ e $\frac{\partial f_{d}(s)}{\partial x_{i}}=0$, para todo $1 \leq i \leq n$. Caso contrário, temos que $S$ não é solução de $(*)$ e por $(2)$ acima temos que grad $f_{d}(s) \neq 0$. Pela equação (1) temos que 
$\operatorname{grad} f_{d}(s)=-x_{n+1}^{0} \operatorname{grad} f_{d-1}(s)$ com $x_{n+1}^{0} \neq 0$ e $\operatorname{grad} f_{d-1}(s) \neq 0$. Portanto, o ponto $R=(s: 1)$ pertence a reta $\overline{P S}$ e $R \in \operatorname{Sing}(f)$.

O Lema 2 de Bruce e Wall em [11] pode ser obtido como corolário do teorema anterior.

Corolário 7.6. i) Seja $F=x_{3}\left(x_{1}^{2}-x_{0} x_{2}\right)+f_{3}\left(x_{0}, x_{1}, x_{2}\right)$ e $G=x_{3}\left(x_{1}^{2}-\right.$ $\left.x_{0} x_{2}\right)+g_{3}\left(x_{0}, x_{1}, x_{2}\right), V=F^{-1}(0)$ e $P=(0: \ldots: 0: 1)$. Temos que $F=0$ $e G=0$ são superfícies cúbicas projetivamente equivalentes fixando $P$, se $e$ somente se, $f_{3}\left(\theta^{2}, \theta \phi, \phi^{2}\right)$ e $g_{3}\left(\theta^{2}, \theta \phi, \phi^{2}\right)$ são sêxticas binárias equivalentes.

ii) Para cada singularidade $Q \neq P$ de $F$, a reta $\overline{P Q}$ está em $V$, então temos uma raiz comum de $f_{2}$ e $f_{3}$, isto é, $f_{3}\left(\theta^{2}, \theta \phi, \phi^{2}\right)=0$, cuja multiplicidade é maior ou igual a 2.

iii) Cada raiz determina uma reta $l$ passando por $P$ em $V$. Se a raiz tem multiplicidade maior ou igual a 2, existe precisamente um outro ponto singular em $l$.

Considerado o caso em que $g$ é uma interseção completa, temos pelo teorema de Bezout que a solução de $f_{d}=f_{d-1}=0$ são $(d-1) d$ retas passando pela origem, contadas com as respectivas multiplicidades.

Para polinômios em $\mathbb{C}^{3}$ de grau maior que 3 , temos o seguinte corolário.

Corolário 7.7. Seja $f\left(x_{0}, x_{1}, x_{2}\right)=f_{d-1}\left(x_{0}, x_{1}, x_{2}\right)+f_{d}\left(x_{0}, x_{1}, x_{2}\right)$.

(i) Se $g=\left(f_{d-1}, f_{d}\right)$ é ICIS, então $f$ é regular no infinito e $X$ é uma curva formada por $(d-1) d$ retas simples.

(ii) Se $g$ não é ICIS, então $X=g^{-1}(0)$ contém alguma reta múltipla. A classificação de $X$ é dada pelas partições $\left(l_{1}^{a_{1}}, \ldots, l_{s}^{a_{s}}\right)$ tais que $\sum_{i=1}^{s} a_{i}=$ 
$(d-1) d$

Nota 7.8. Se o feixe é do tipo $a^{l}$, dizemos que o feixe tem l retas de multiplicidade a.

\subsection{Superfícies em $\mathbb{C}^{3}$ com defeito de Betti mínimo e máximo}

Nesta seção vamos analisar em quais condições $f: \mathbb{C}^{3} \rightarrow \mathbb{C}, f\left(x_{0}, x_{1}, x_{2}\right)=$ $f_{d-1}\left(x_{0}, x_{1}, x_{2}\right)+f_{d}\left(x_{0}, x_{1}, x_{2}\right)$ possui defeito de Betti mínimo que vamos indicar por $\Delta_{m i n}$, e quando possui defeito de Betti máximo, que indicaremos por $\Delta_{\text {max }}$, para um tipo especial de singularidade no infinito que chamaremos de nó generalizado.

Definição 7.9. Um ponto singular no infinito $Q$ de $f$ é um nó generalizado, se $Q$ é a única singularidade no infinito de $f$, e é uma singularidade do tipo $A_{1}$ de $f_{d}$.

Como $Q$ é o único ponto singular no infinito, podemos supor sem perda de generalidade que $Q=(0: 0: 1: 0)$. Sendo $Q$ um nó generalizado podemos considerar $f=f_{d-1}+f_{d}$, em que

(*) $\quad f_{d-1}\left(x_{0}, x_{1}, x_{2}\right)=x_{2}^{d-2} p_{1}\left(x_{0}, x_{1}\right)+x_{2}^{d-3} p_{2}\left(x_{0}, x_{1}\right)+\ldots+x_{2} p_{d-2}\left(x_{0}, x_{1}\right)+$ $p_{d-1}\left(x_{0}, x_{1}\right), p_{i}$ é um polinômio homogêneo de grau $i$ nas variáveis $x_{0}$ e $x_{1}$ com $p_{1}\left(x_{0}, x_{1}\right)=a x_{0}+b x_{1}, \mathrm{e}$

$$
(* *) \quad f_{d}\left(x_{0}, x_{1}, x_{2}\right)=q_{d}\left(x_{0}, x_{1}\right)+\sum_{i=1}^{d-3} x_{2}^{i} q_{d-i}\left(x_{0}, x_{1}\right)+x_{2}^{d-2} x_{0} x_{1},
$$

$q_{j}$ é um polinômio homogêneo nas variáveis $x_{0}$ e $x_{1}$ de grau $j$.

Lema 7.10. Se $Q$ é um nó generalizado de $f=f_{d-1}+f_{d}$, então genericamente $Q$ é uma singularidade $A_{k}$ ou é uma singularidade não isolada de tipo $A_{\infty}$. 
Demonstração. Podemos tomar $Q=(0: 0: 1: 0)$ e é imediato da definição de nó generalizado que o posto da Hessiana de $F$ no ponto $Q=(0: 0: 1: 0)$ é 2 ou 3 o que garante que a singularidade é de tipo $A_{k}$, com $1 \leq k \leq \infty$.

Lema 7.11. Seja $Q$ nó generalizado de $f=f_{d-1}+f_{d}$. Então $Q$ é uma singularidade $A_{k}, k \geq 2$ se e somente se a multiplicidade da interseção de $\left\{f_{d}=0\right\}$ e $\left\{f_{d-1}=0\right\}$ em $x_{2}=1$ é $k+1$.

Demonstração. Sejam $f_{d-1}$ e $f_{d}$ como em $(*)$ e $(* *)$. Como antes $p_{1}\left(x_{0}, x_{1}\right)=$ $a x_{0}+b x_{1}$. Se $a b \neq 0$, é fácil verificar que $Q$ é de tipo $A_{1}$.

Se $a=b=0$, a singularidade em $Q$ é não isolada, conforme justificamos na Observação 7.12 .

Se $(a, b) \neq(0,0)$, podemos supor, sem perda de generalidade que $b=0$ e $a \neq 0$. Então, fazendo $x_{2}=1$, obtemos

$$
\begin{gathered}
F\left(x_{0}, x_{1}, 1, x_{3}\right)=x_{3}\left(a x_{0}+p_{2}\left(x_{0}, x_{1}\right)+\ldots+p_{d-1}\left(x_{0}, x_{1}\right)\right) \\
+\mathrm{q}_{d}\left(x_{0}, x_{1}\right)+\sum_{i=1}^{d-3} q_{d-i}\left(x_{0}, x_{1}\right)+x_{0} x_{1} .
\end{gathered}
$$

Por mudanças de coordenadas, obtemos

$$
F\left(x_{0}, x_{1}, 1, x_{3}\right) \approx x_{3} x_{0}+\widetilde{q}_{d}\left(x_{0}, x_{1}\right)+\sum_{i=1}^{d-3} \widetilde{q}_{d-i}\left(x_{0}, x_{1}\right)+x_{0} x_{1} .
$$

Fatorando $x_{0}$ na expressão acima, temos:

$$
F\left(x_{0}, x_{1}, 1, x_{3}\right) \approx x_{0}\left(x_{3}+S\left(x_{0}, x_{1}\right)\right)+\widetilde{q}_{d}\left(0, x_{1}\right)+\sum_{i=1}^{d-3} \widetilde{q}_{d-i}\left(0, x_{1}\right) .
$$

em que grau de $S \geq 2$. Portanto

$$
F\left(x_{0}, x_{1}, 1, x_{3}\right) \approx x_{3} x_{0}+\Phi\left(x_{1}\right), \operatorname{grau} \Phi \geq 3 .
$$

Nesta forma normal, é fácil ver que a multiplicidade de interseção de $\left\{f_{d}=0\right\}$ e $\left\{f_{d-1}=0\right\}$ em $x_{0}=x_{1}=x_{3}=0$ e $x_{2}=1$ é $k+1$, se e somente se, $Q$ é uma singularidade $A_{k}$. 
Observação 7.12. Se $p_{1}\left(x_{0}, x_{1}\right)=a x_{0}+b x_{1} \equiv 0$, isto é, $a=b=0, a$ singularidade é não isolada e de tipo $A_{\infty}$. De fato, se $a=b=0$, Verifica-se que $x_{0}=x_{1}=0$ é singularidade não isolada de $F\left(x_{0}, x_{1}, 1, x_{3}\right)=0$, pois a seguinte inclusão $\left\{\left(0: 0: x_{2}: x_{3}\right) ; x_{2}, x_{3} \in \mathbb{C}\right\} \subseteq \operatorname{Sing}(f)$ é verdadeira. As seções afins $x_{1}=0$ são sempre $A_{1}$ e se $x_{2}=0$, para $p$ e q genéricos, a singularidade tem número de Milnor $\mu\left(p_{d-1}\right)=(d-2)(d-2)$. Como o conjunto crítico suave é 1-dimensional, temos uma "line singularities"e em condições genéricas, temos sempre uma singularidade não isolada do tipo $A_{\infty}$

Teorema 7.13. Suponha que $Q$ é o único ponto singular no infinito de $f$. As seguintes condições são equivalentes:

(1) Q é um nó generalizado e a singularidade no infinito de $f$ é do tipo $A_{1}$.

(2) $\Delta_{2}(f)=\Delta_{\min }$

(3) O feixe $\left(f_{d-1}, f_{d}\right)$ é do tipo $1^{d(d-1)-2} 2$.

Demonstração. Primeiramente vamos provar a equivalência dos itens (1) e (2). Da fórmula 6.5 para calcular o defeito de Betti, temos que

$$
\Delta_{2}(f)=\mu_{Q}\left(\overline{X_{0}}\right)+\mu_{Q}\left(\overline{X_{0} \cap H^{\infty}}\right) .
$$

Como $Q$ é um nó generalizado, temos que $\mu_{Q}\left(\overline{X_{0} \cap H^{\infty}}\right)=1$. Por outro lado, como $Q$ é uma singularidade do tipo $A_{1}$ de $f$, temos que $\mu_{Q}\left(\overline{X_{0}}\right)=1$, logo $\Delta_{2}(f)=2$. Além disso, podemos observar que o defeito de Betti é mínimo, pois caso contrário $f$ seria regular no infinito. Reciprocamente, se $Q$ não for um nó generalizado segue que $\mu_{Q}\left(\overline{X_{0} \cap H^{\infty}}\right)>1$. Como $f$ não é regular no infinito segue que $\mu_{Q}\left(\overline{X_{0}}\right) \geq 1, \operatorname{logo} \Delta_{2}(f) \geq 3$. 
$2 \Leftrightarrow 3$ Como estamos analisando no ponto $Q=(0: 0: 1: 0)$, vamos fazer $x_{2}=1$ em $F\left(x_{0}, x_{1}, x_{2}, x_{3}\right)=x_{3} f_{d-1}\left(x_{0}, x_{1}, x_{2}\right)+f_{d}\left(x_{0}, x_{1}, x_{2}\right)$, em que $f_{d-1}$ e $f_{d}$ são como em $(*)$ e $(* *)$. Como $Q$ é uma singularidade de tipo $A_{1}$ de $F\left(x_{0}, x_{1}, 1, x_{3}\right)$, temos que $a . b \neq 0$, o que implica que a multiplicidade da reta que liga o ponto $P=(0: 0: 0: 1)$ com o ponto $Q=(0: 0: 1: 0)$ é 2. Agora queremos verificar o que acontece fora do ponto $Q=(0: 0: 1: 0)$. Sabemos pelo Teorema de Bezout que $f_{d}$ intersecta $f_{d-1}$ em $d(d-1)$ retas contadas com multiplicidades, como já temos uma reta dupla no ponto $Q$, resta verificar que existem $d(d-1)-2$ retas simples fora do ponto $Q$. É fácil verificar que se $x_{2}=0$ em $\left\{f_{d-1}=0\right\} \cap\left\{\frac{\partial f_{d}}{\partial x_{j}}, j=1, \ldots, n\right\}$, em que $f_{d-1}$ e $f_{d}$ são como em $(*)$ e $(* *)$ respectivamente, então $x_{0}=x_{1}=0$. Por outro lado se $x_{2}=1$ e $x_{0} \neq 0$ ou $x_{1} \neq 0$, para polinômios genéricos temos $f_{d-1}$ e $f_{d}$ são transversais, logo temos $d(d-1)-2$ retas simples. Portanto $\Delta_{\min }=2$, se e somente se, o feixe $\left(f_{d-1}, f_{d}\right)$ é do tipo $1^{d(d-1)-2} 2$, isto é, uma reta dupla e $d(d-1)-2$ retas simples.

Na prova do teorema acima observamos que a singularidade no infinito do nó generalizado é do tipo $A_{1}$ se e somente se, $a . b \neq 0$ em $p_{1}\left(x_{0}, x_{1}\right)=$ $a x_{0}+b x_{1}$. Além disso, se $a=b=0$ a singularidade é não isolada e é de tipo $A_{\infty}$, pela Observação 7.12. A seguir, quando $a \neq 0$ e $b=0$, ou viceversa, vamos determinar condições necessárias e suficientes para que o feixe $g=\left(f_{d-1}, f_{d}\right)$ de um nó generalizado de $f=f_{d-1}+f_{d}$ seja de tipo $1^{d(d-2)} d$.

Teorema 7.14. Suponha que $Q$ é o único ponto singular isolado no infinito de $f$ e é um nó generalizado. As seguintes condições são equivalentes:

(1) A singularidade no infinito é do tipo $A_{d-1}$ de $f$. 
(2) $\Delta_{2}(f)=d$.

(3) O feixe $\left(f_{d-1}, f_{d}\right)$ é do tipo $1^{d(d-2)} d$.

Demonstração. $1 \Leftrightarrow 2$ Da fórmula 6.5 para calcular o defeito de Betti, temos que

$$
\Delta_{2}(f)=\mu_{Q}\left(\overline{X_{0}}\right)+\mu_{Q}\left(\overline{X_{0} \cap H^{\infty}}\right) .
$$

Como $Q$ é um nó generalizado, temos que $\mu_{Q}\left(\overline{X_{0} \cap H^{\infty}}\right)=1$. Por outro lado, sendo $Q$ uma singularidade do tipo $A_{d-1}$ de $f$, obtemos $\mu_{Q}\left(\overline{X_{0}}\right)=d-1$, logo $\Delta_{2}(f)=d$. Além disso, podemos observar que para valores genéricos, pelo Lema 7.10, a singularidade é no tipo $A_{k}, 2 \leq k \leq d$. Reciprocamente suponhamos que $\Delta_{2}(f)=d$. Como $Q$ é um nó generalizado, $\mu_{Q}\left(\overline{X_{0} \cap H^{\infty}}\right)=1$, então por 6.5 obtemos $\mu_{Q}\left(\overline{X_{0}}\right)=d-1$. Como a singularidade no infinito é de tipo $A_{k}, Q$ é portanto uma singularidade de tipo $A_{d-1}$.

$2 \Leftrightarrow 3$ Como estamos analisando no ponto $Q=(0: 0: 1: 0)$, vamos fazer $x_{2}=1$ em $f\left(x_{0}, x_{1}, x_{2}\right)$. Como $Q$ é uma singularidade isolada de $f$, podemos supor que $a \neq 0$ e $b=0$, e ainda que a singularidade é do tipo $A_{d-1}$. Pelo Lema 7.11, a multiplicidade da reta que liga o ponto $P=(0: 0: 0: 1)$ com o ponto $Q=(0: 0: 1: 0)$ é $d$. Agora queremos verificar o que acontece fora do ponto $Q=(0: 0: 1: 0)$. Sabemos pelo teorema de Bezout que $f_{d}$ intersecta $f_{d-1}$ em $d(d-1)$ retas, como já temos uma reta com multiplicidade $d$ no ponto $Q$, resta verificar que existem $d(d-1)-d$ retas simples fora do ponto $Q$. É fácil verificar que se $x_{2}=0 \mathrm{em}\left\{f_{d-1}=0\right\} \cap\left\{\frac{\partial f_{d}}{\partial x_{j}}, j=1, \ldots, n\right\}$, em que $f_{d-1}$ e $f_{d}$ são como em $(*)$ e $(* *)$ respectivamente, então $x_{0}=x_{1}=0$. Por outro lado se $x_{2}=1$ e $x_{0} \neq 0$ ou $x_{1} \neq 0$, para polinômios genéricos $f_{d-1}$ e $f_{d}$ são transversais, logo temos $d(d-1)-d$ retas simples. Portanto 
acabamos de provar que $\Delta_{2}(f)=d$, se e somente se, o feixe $\left(f_{d-1}, f_{d}\right)$ é do tipo $1^{d(d-2)} d$, isto é, uma reta com multiplicidade $d$ e $d(d-2)$ retas simples. 


\section{Referências Bibliográficas}

[1] V.I. ARnold, Critical points of functions on a manifold with boundary the simple Lie groups $B_{k}, C_{k}, F_{4}$ and singularities of evolutes, Uspekhi Mat. Nauk 33 , no 5(203), (1978).

[2] V.I. ARNold, Singularities of fractions and behaviour of polynomials at infinity.

[3] E. Artal, I. Luengo, A. Melle, On the topology of a generic fibre of a polynomial function, Communications in Algebra, 28(4), (2000), 17671787.

[4] R. Benedetti, J.J. Risler, Real algebraic and semialgebraic sets, Actualités Mathématiques, Hermann, Éditeurs Des Scienses et des Arts, (1990).

[5] F. Bobadilla, Relative Morsification theory, Topology, 43, (2004), 925982.

[6] J. Briançon, P. Maisonobe, Localisation de systèmes différtieles, stratifications de Whitney et condition de Thom, Invent. Math 117: (1994), 531-550.

[7] B. Egbert And K. Horst, Plane algebraic curves, Birkhauser Verlag Basel (1986). 
[8] S.A. Broughton, Milnor number and the topology of polynomial hypersurface, Inventiones Math. 92 (1988), 217-241.

[9] S.A. Broughton, On the topology of polynomial hypersurfaces, Proceedings A.M.S Symp. in Pure Math.,vol. 40, I (1983), 165-178.

[10] J. W. BRuCE, Classifications in singularity theory and their applications, New Developments in Singularity Theory(Cambridge, 2000) 3-33, Nato, Sci. Ser. II Math. Phys. Chem. 21 Kluwer. Acad. Publ., (2001).

[11] J.W. Bruce And C. T. C. Wall, On The Classification of Cubic Surfaces, J.London Math. Soc(2) 19 (1979), 245-256.

[12] A. DimcA, Singularities and topology of hypersurfaces, Springer-Verlag, (1992).

[13] A. H. Durfee, Five definitions of critical point at infinity, Progress in Math. vol. 162, (1998).

[14] C. Ehresmann, Les connexions infinitésimales dans un espace fibré différentiable, Colloque de Topologie Bruxelles, (1950), 29-55.

[15] C. G. GiBson, Elementary geometry of algebraic curves: Undergraduate Introduction Cambridge University, Cambridge (1998).

[16] C. G. Gibson, Singular points of smooth mappings, Research notes in Mathematics, Pitman(Advanced Publishing Program), Boston, Mass (1979).

[17] M. Golubitsky And Guillemin, Stable mappings and their singularities, GTM. 14, Springer-Verlag, (1973). 
[18] M. Goresky, R. MacPherson, Stratified Morse theory, Springer Verlag, Berlin-Heidelberg-New York (1987).

[19] H. Hamm And D. T. Lê, Rectified homotopical depth and Grothendieck conjectures, in:P. Cartier et all.(eds) Grothendieck Festschrift II, Birkhauser (1991), 311-351.

[20] H. Hamm And D. T. LêE, Sur la topologie de polinômes complexes, Acta Math. Vietnamica 9 (1984), 21-32.

[21] D.T. Lê , Calcul du Nombre de Cycles Èvanouissants D'une Hypersurfaces Complexe, Ann. Inst. Fourrier, Grenoble, 23, 4, (1973) 261-270.

[22] D. T. LêE, Complex analytic functions with isolated singularities, J. Algebraic Geometry, 1 (1992), 83-100.

[23] D. T. Lê, Some remarks on the relative monodromy, in: real and complex singularities, Oslo (1976), Sijhoff em Norhoff, Alphen a.d Rijn (1977), 397-403.

[24] D.T. Lê, Weber, Polynômes à fibres rationelles et conjecture jacobienne à 2 variables, C.R Math. Acad. Sci. Paris, t. 320, Série I (1995), 581-584.

[25] S. Lojasievicz, Ensembles semi-analytiques, IHES Mathematics, (1965).

[26] E. J. N. LOOIJENGA, Isolated singular points on complete intersections, London Mathematical Society Lecture Note Series: 77.

[27] B. MARTin, Milnor algebras could be isomorphic to modular algebras, Journal of Symbolic Computation 44, (2009) 1268-1279. 
[28] J. MarTinet, Singularities of smooth functions and mappings, LMS, Lecture Notes 58, Cambridge Universty Press, (1982).

[29] J. N. Mather, Notes on topological stability, Lectures notes, Harvard Auniversity, (1970).

[30] J.W. Milnor, Singular points of Complex Hypersurfaces, Ann. of Math. Studies 61, Princeton (1968).

[31] J. W. Milnor, Morse teory, Annals of Mathematics Studies, Princeton, Univ. Press 51, (1963).

[32] A. Parusinski, A note on singularities at infinity of complex polynomials. Symplectic Singularities and Geometry of Gauge Fields, Banach Center Publications volume 39 Institute of Mathematics, Polish Academy Of Sciences, Warszawa (1997).

[33] A. Parusinski, On the bifurcation set of complex polynomials with isolated singularities at infinity, Compositio Mathematica, tome $97, \mathrm{n}^{\circ}$ 3 (1995), 369-384.

[34] R. PellikaAn, Finite determinacy of functions with non-isolated singularities, Proc. London. Math. Soc. (3), 57, (1988), 357-382.

[35] F. Pham, Vanishing homologies and the $n$ variable saddle point method, Arcata Proc. of Symp. in Pure Math. vol. 40, II (1983), 319-333.

[36] M.A. S. RuAs, Singularidades de aplicações diferenciàveis, Notas Didáticas do ICMC, (2008).

[37] B. SEgRE, The nonsingular cubic surfaces, O.U.P, (1942). 
[38] D. Siersma and J. Smeltink, Classification of singularities at infinity of polynomials of degree 4 in two variables, Georgian Mathematical Journal, Vol. 7 , Number 1, (2000), 179-198.

[39] D. Siersma, M. Tibar, Betti bounds of polynomials, Moscow Mathematical Journal, Vol 11, no 3 (2011), 599-615.

[40] D. Siersma E M. Tibar, Singularities at infinity and their vanishing cycles, Duke Math. Journal 80 (3) (1995), 771-783.

[41] R. Switzer ,Algebraic topology-homotopy and homology. SpringerVerlag, Berli-Heidelberg-New York (1975).

[42] F. TARI, Singularidades de aplicações diferenciáveis, Notas Didáticas do ICMC, 34, (1999).

[43] T. DE Jong, Some classes of line singularities, Math. Z. 198, (1988), 497-517.

[44] R. Tном, Ensembles et morphismes stratifiés, Bull. Amer. Math. Soc., 75 (1969), 249-312.

[45] M. Tibar And D. Siersma, Singularity exchange at infinity, arXiv.Math. AG/0401396 V1 28 Jan (2004).

[46] M. TibAR, Fibrations of analytic maps, Lectures Notes of a Short Course in ICMC, São Carlos, June (2010).

[47] M. TIBAR, Lectures on singularities of polynomials, São Carlos, May (2007). 
[48] M. TIBAR, Polynomials and vanishing cycles, Université des Sciences et Technologies de Lille France, (2007).

[49] M. TiBAR, On the monodromy fibration of polynomial functions with singularities at infinity, C.R. Acad. Sci. Paris, 324 (1997), 1031-1035.

[50] M. TiBAR, Regularity at infinity of real and complex polynomial maps, Singularity Theory, the C.T.C. Wall Anniversary Volume, LMS Lecture Notes Series 263 (1999), 249-264. Cambridge University Press.

[51] C.T.C. WALL, Affine cubic functions II- functions on $\mathbb{C}^{3}$-with a corank 2 singular point, Topology vol. 19, (1980), 89-98.

[52] C.T.C. Wall, Affine cubic functions III- the real plane, Math. Proc. Camb. Phil. Soc., 87, 1 (1980).

[53] C.T.C. WALL, Affine cubic functions $I V$ - functions on $\mathbb{C}^{3}$ nonsingular at infinity, A. Math. and Physical Sciences- vol 302, n 1470, (1981), 415455 .

[54] H. Whitney, Local properties of analytic varieties, differential and combinatorial topology, Princeton, Univ. Press, (1965), 205-244.

[55] H. Whitney, Tangents to an analytic variety, Annals of Mathematics, 81, (1965), 496-549.

[56] A. ZAharia, Characterizations of simple isolated line singularities, Canad. Math. Bull. Vol. 42(4), (1999) 499-506. 